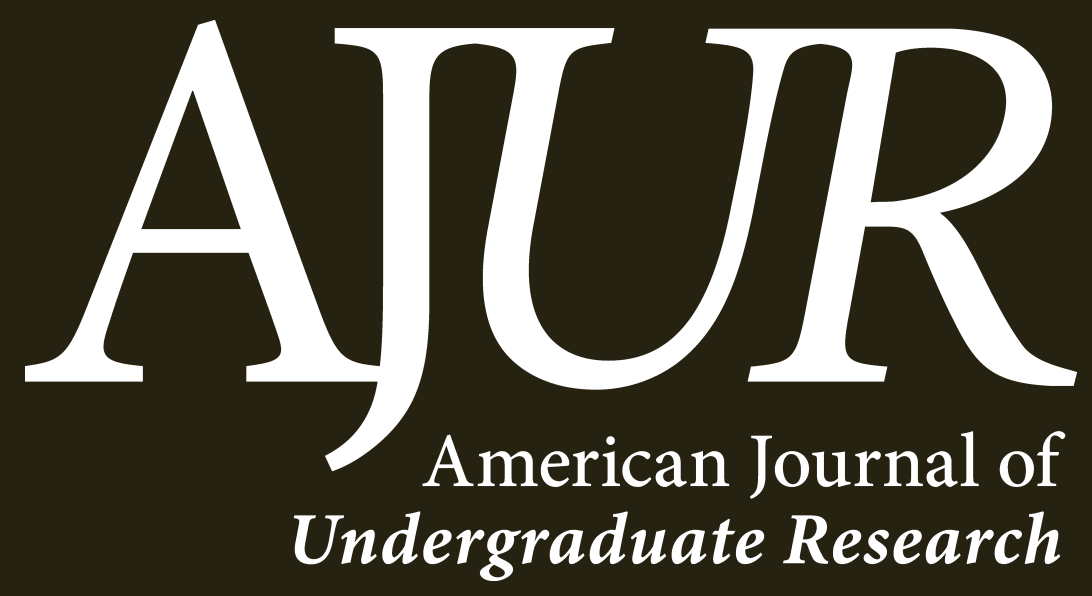

Volume 13 | Issue 3 | August 2016 www.ajuronline.org 


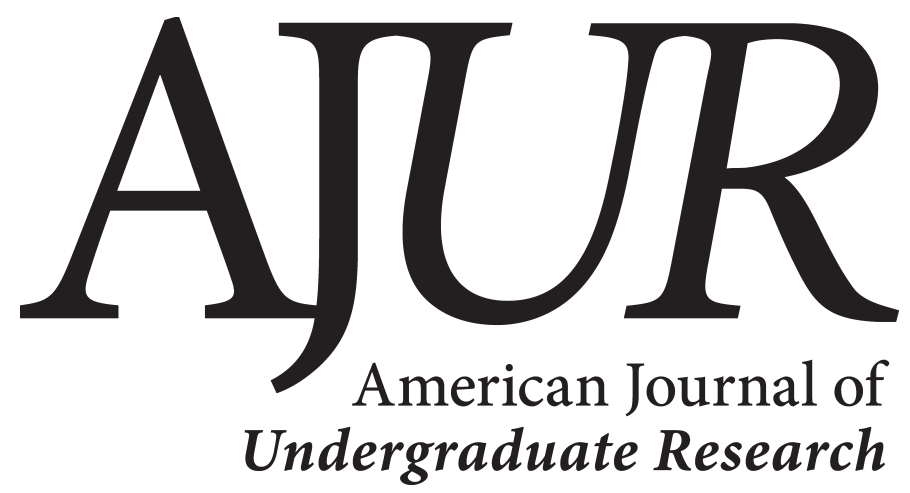

Volume 13 | Issue 3 | August 2016

www.ajuronline.org

AJUR History and Editorial Board

Effects of the Electronegativity of Second Row Elements on Their Bonding to Boron

Xavier Shiu \& J. Conceicao

What Angle Will You Take? Patterns of Perspective-Taking in a Body-

Based Task

Tegan Garon

Affine Symmetry Tensors in Minkowski Space

Isaac Ahern \& Sam Cook

33 A Relationship Between Time Perception and State-Anxiety

James Brown

41 On Closure Properties of Irrational and Transcendental Numbers under Addition and Multiplication

Shekh Mohammed Zahid \& Prasanta Kumar Ray

45 Peaked T-Waves and Potassium: The Reliability of Peaked T-Waves as Indicators of Hyperkalemia in End-Stage Renal Patients Undergoing Hemodialysis

Ryan D. Pappal, Ilse M. Espina \& Zubaid Rafique

Modeling Internet Traffic Generations Based on Individual Users and Activities for Telecommunication Applications

Sara Stoudt, Pamela Badian-Pessot, Blanche Ngo Mahop, Erika Earley, Jordan Menter, Yadira Flores, Danielle Williams, Weijia Zhang, Liza Maharjan, Yixin Bao, Laura Rosenbauer, Van Nguyen, Veena Mendiratta \& Nessy Tania 
American Journal of Undergraduate Research (AJUR) is a national, peer-reviewed, open-source, quarterly, multidisciplinary student research journal. It is indexed internationally by EBSCO, and is listed via the Library of Congress under ISSNs of 1536-4585 (for print) and 2375-8732 (for web). The journal was established in 2002.

EDITORIAL TEAM Volume 13 | Issue 3 | August 2016

Dr. Kestutis G. Bendinskas, Editor, editor@ajuronline.org Dr. Anthony Contento, Assistant Technical Editor, technicaleditor@ajuronline.org Rose Throop, Art and Copy Editor,copyeditor@ajuronline.org

Daniel Laird, Web Master,webmaster@ajuronline.org Bonita Graham, LaTex Editor

\section{EDITORIAL BOARD by subject area}

ACCOUNTING

Dr. Dean Crawford, dean.crawford@oswego.edu

\section{ART HISTORY}

Dr. Lisa Seppi, lisa.seppi@oswego.edu

ASTROPHYSICS

Dr. Shashi Kanbur, shashi.kanbur@oswego.edu

BEHAVIORAL NEUROSCIENCE

Dr. Aileen M. Bailey, ambailey@smcm.edu

\section{BIOCHEMISTRY}

Dr. Pamela K. Kerrigan, pamela.kerrigan@mountsaintvincent.edu

\section{BIOENGINEERING}

Dr. Jorge I. Rodriguez, jorger@clemson.edu

BIOINFORMATICS

Dr. Kevin Daimi, daimikj@udmercy.edu

Dr. John R. Jungck, jungck@udel.edu

Dr. Isabelle Bichindaritz, ibichind@oswego.edu

\section{BIOLOGY, PHYSIOLOGY}

Dr. David Dunn,

david.dunn@oswego.edu

BIOLOGY, DEVELOPMENTAL

Dr. Poongodi Geetha-Loganathan, p.geethaloganathan@oswego.edu

\section{BIOLOGY, MICROBIOLOGY}

Dr. Peter Newell, peter.newell@oswego.edu

\section{BOTANY}

Dr. William R. Bromer, wbromer@stfrancis.edu

Dr. Julien Bachelier, julien.bachelier@oswego.edu

\section{CHEMISTRY}

Dr. Alfredo Castro,

castroa@felician.edu

Dr. Charles Kriley,

cekriley@gcc.edu

Dr. Douglas Mulford, douglas.mulford@emory.edu

Dr. Mark A. Benvenuto,

benvenma@udmercy.edu

COMMUNICATION STUDIES

Dr. Jennifer Gerometta, jgerometta@iona.edu

COMPUTER SCIENCES

Dr. Dele Oluwade,

deleoluwade@yahoo.com

Dr. Kevin Daimi,

daimikj@udmercy.edu

\section{ECOLOGY}

Dr. William R. Bromer,

wbromer@stfrancis.edu

\section{ECONOMICS}

Dr. Elizabeth Schmitt, elizabeth.schmitt@oswego.edu

EDUCATION, PHYSICS

Dr. Andrew D. Gavrin,

agavrin@iupui.edu

ENGINEERING, ELECTRICAL

Dr. Michael Omidiora,

michael.omidiora@nyu.edu

ENGINEERING, ENVIRONMENTAL

Dr. Eileen M. Cashman, eileen.cashman@humboldt.edu

ENGINEERING, MANUFACTURING AND CONSTRUCTION, ROBOTICS

Dr. Haoyu Wang,

wanghao@mail.ccsu.edu

\section{ENGINEERING, SOFTWARE}

Dr. Kevin Daimi,

daimikj@udmercy.edu

ENVIRONMENTAL SCIENCE

Dr. Eileen M. Cashman, eileen.cashman@humboldt.edu

GEOLOGY

Dr. Larry Davis,

ldavis@csbsju.edu

HISTORY

Dr. Richard Weyhing, richard.weyhing@oswego.edu

Dr. Murat Yasar,

murat.yasar@oswego.edu

HONORARY EDITORIAL BOARD MEMBER

Dr. Lorrie Clemo,

lorrie.clemo@oswego.edu

KINESIOLOGY / EXERCISE SCIENCE

Dr. David Senchina,

david.senchina@drake.edu

LITERARY STUDIES

Dr. Douglas Guerra,

douglas.guerra@oswego.edu

\section{MATHEMATICS}

Dr. John Emert,

emert@bsu.edu

Dr. Jeffrey J. Boats,

boatsjj@udmercy.edu

Dr. J.D. Phillips,

jophilli@nmu.edu

Dr. Dele Oluwade,

deleoluwade@yahoo.com

Dr. Christopher Baltus,

christopher.baltus@oswego.edu

Dr. Mark Baker,

mark.baker@oswego.edu
METEOROLOGY

Dr. Steven Skubis, steven.skubis@oswego.edu

\section{NANOSCIENCE}

Dr. Gary Baker, bakergar@missouri.edu

NEUROSCIENCE

Dr. Pamela E. Scott-Johnson, pamela.scottjohnson@morgan.edu

Dr. Amy Overman,

aoverman@elon.edu

PHYSICS

Dr. Carolina Ilie, carolina.ilie@oswego.edu

\section{POLITICAL AND SOCIAL SCIENCES}

Dr. Dean Dohrman, dean.dohrman@csuglobal.edu

\section{PSYCHOLOGY}

Dr. Amy Overman, aoverman@elon.edu

Dr. Pamela E. Scott-Johnson, pamela.scottjohnson@morgan.edu

STATISTICS

Dr. Mark Ecker, mark.ecker@uni.edu

TECHNOLOGY, ENGINEERING

Dr. Recayi Pecen, regpecen@na.edu 


\section{SPECIAL THANKS}

AJUR is made possible through the assistance of our sponsors.

Support for this issue has been provided by Wiley as well as the Office of the Provost at the State University of New York at Oswego. Thank you!
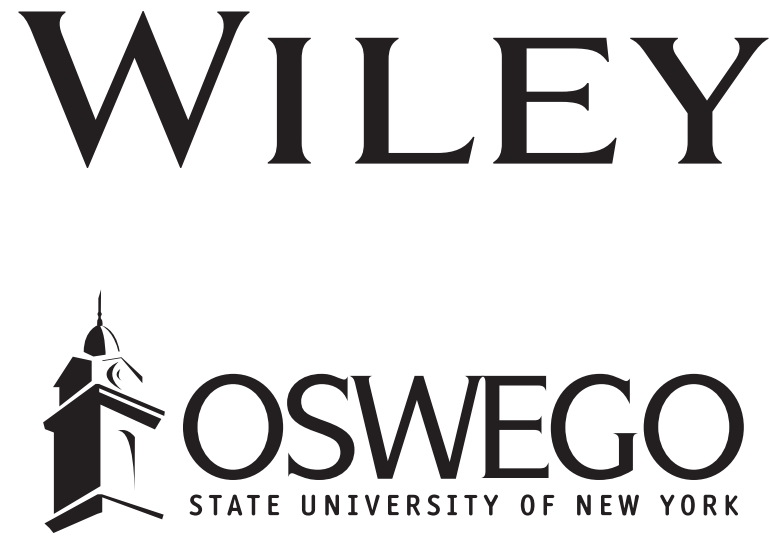

Interested in supporting quality undergraduate research?

Request sponsorship information at editor@ajuronline.org 


\title{
Effects of the Electronegativity of Second Row Elements on Their Bonding to Boron
}

\author{
Xavier Shiu and J. Conceicao* \\ Department of Math \& Natural Sciences, Metropolitan Community College, South Omaha Campus, Omaha, NE
}

Student:xmtwoshoes@gmail.com

Mentor:jconceicao@mccneb.edu*

\begin{abstract}
The effects of electronegativity on the bonding between boron and second row elements are studied in this paper. Calculations using Density Functional Theory (DFT), Moller-Plesset Theory (MP2) and Natural Bonding Orbital (NBO) analysis were performed on $\mathrm{BF}_{3}, \mathrm{~B}(\mathrm{OH})_{3}$ and $\mathrm{B}\left(\mathrm{NH}_{2}\right)_{3}$ and the localized bonding properties of these molecules were elucidated. All of these molecules showed the absence of pi-bonding and did not obey the octet rule. With decreasing electronegativity of the terminal atoms, $\mathrm{F}, \mathrm{O}$ and $\mathrm{N}$ in $\mathrm{BF}_{3}, \mathrm{~B}(\mathrm{OH})_{3}, \mathrm{~B}\left(\mathrm{NH}_{2}\right)_{3}$ there is increasing the propensity of electron donation from these terminal atoms to the empty p-orbital of the central boron. Within the $\mathrm{BH}_{2}-\mathrm{F}, \mathrm{BH}_{2}-\mathrm{OH}$ and $\mathrm{BH}_{2}-\mathrm{NH}_{2}$ series, the amino-borane showed the largest change in relative bond length and angle across this set. Furthermore, the borate anion, ${ }^{-} \mathrm{O}-\mathrm{B}(\mathrm{OH}) 2$ was subjected to identical analysis and pi-bond formation was observed. Our results show that a good match orbital energies between the donor and acceptor orbitals are important for pi-bond formation.
\end{abstract}

\section{KEYWORDS}

Electronegativity; Boron Trifluoride; Boric Acid; Triaminoborane; Borate Anion; Octet Rule; Density Functional Theory; Natural Bonding Orbital; Pi-Bond; Double Bond

\section{INTRODUCTION}

Chemical bonding is one of the central ideas in chemistry and the octet rule has been used successfully to describe the bonding and reactions in a wide range of substances and this applies to much of organic chemistry and other molecular compounds of the second row elements. ${ }^{1}$ This rule state that elements react to form compounds by sharing their valence electrons and stability is achieved when each element has a total of eight valence electrons. (Hydrogen and lithium are exceptions and only requires two). Despite these successes, a number of covalent compounds do not obey this rule. Examples of this violation given in undergraduate-level texts frequently list compounds of beryllium, boron, and elements of the third row. ${ }^{2,3}$ To our end, $\mathrm{BF}_{3} \mathrm{was}$ the model example and is the catalyst for our study in this paper. The Lewis structure of this molecule and the associated resonance forms are presented as four structures in Figure 1 with the non-octet left most structure followed by three resonance structures that obey the octet rule. ${ }^{1}$

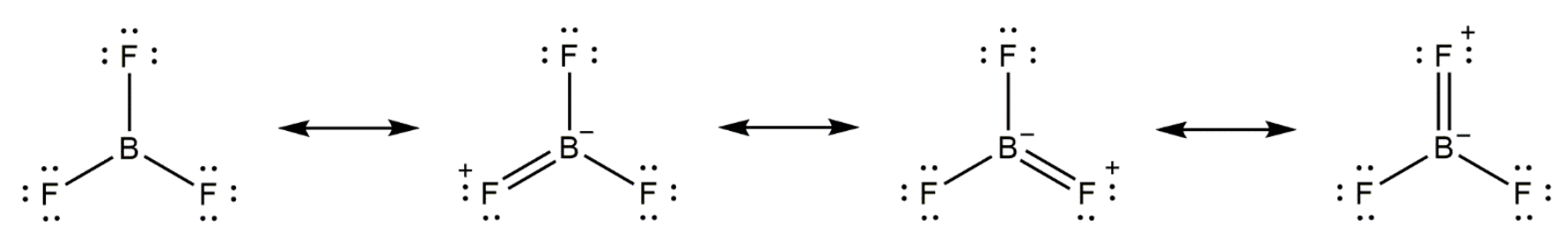

Figure 1. Lewis structure of $\mathrm{BF}_{3}$ and associated resonance forms. 
A possible reason for the absence of octet in boron of $\mathrm{BF}_{3}$ may be that the electronegativity (EN) of the terminal fluorine atoms affects their pi-donor strength, i.e. their willingness to donate an electron pair to form a pi-bond to boron. ${ }^{4}$ In general, if electron pair donation (pi-bond formation) were to happen between an atom of higher EN to one of lower EN, for example from fluorine

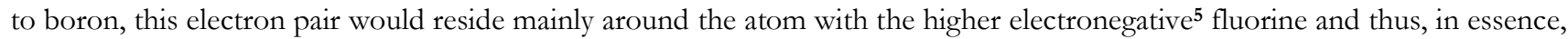
may result in the absence of a pi-bond. Indeed, the effects of $\mathrm{EN}$ was extensively studied in the boron halides, $\mathrm{BF}_{3}$ and $\mathrm{BCl}_{3}$ in relation to their behavior as Lewis acids. ${ }^{5}$ These studies though were conducted with terminal atoms of elements down the same group; these atoms have substantial differences in their atomic size and may give rise to other effects. ${ }^{6}$ It is hoped that this study would add to the extensive studies on boron compounds. ${ }^{7,8,9}$

In this paper the above hypothesis was tested by replacing the terminal atom with elements across a period, i.e. in order to test the effect of EN of terminal atoms on their ability to form pi-bond to the central B atom, fluorine (F) was replaced with the less EN atoms oxygen $(\mathrm{O})$ and nitrogen $(\mathrm{N})$ and these molecules are shown in Figure 2. This approach of using elements across the periodic table has the advantage of bypassing any potential atom size effect as the latter changes slowly across a row. Theoretical calculations, DFT with NBO analysis, were performed to determine the bonding nature between $\mathrm{O}$ and $\mathrm{N}$ respectively to $\mathrm{B}$ in these molecules. With the decreasing $\mathrm{EN}$ of $\mathrm{O}$ and $\mathrm{N}$, it is anticipated that there will be an increasing tendency of electron donation and potential pi-bond formation to boron. Furthermore, since B, N, O and F are in the same period and thus of similar size, it is anticipated that there will be better overlap and more efficient electron donation potentially leading to better bonding. ${ }^{10}$ Although the lack of double bond formation in $\mathrm{BF}_{3}$ can be rationalized in terms of formal charge, EN was chosen as our starting hypothesis because EN has physical origins in the atomic property of nuclear screen and thus effective nuclear charge, ${ }^{11}$ a concept that is more amenable to theoretical studies.
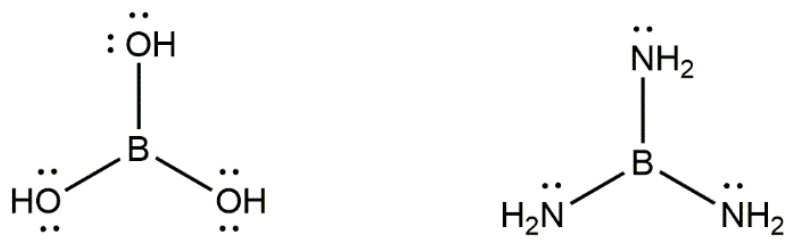

Figure 2. Lewis structures of $\mathrm{H}_{3} \mathrm{BO}_{3}$ and $\mathrm{B}\left(\mathrm{NH}_{2}\right)_{3}$.

\section{METHOD AND PROCEDURES}

Geometry optimization - Theoretical calculations were performed using Gaussian 09W. ${ }^{12}$ Density functional theory (DFT) with the B3LYP functional using the $6-31+G$ and $6-311+G(d, p)$ basis sets as well as Moller Plesset Theory (MP2) using 6-311+G (d, p) basis set were used to optimize the molecular geometry.

Bonding analysis - Natural bonding orbital (NBO) calculations, version 3.1 of this program, were then performed (DFT-B3LYP using the $6-31+\mathrm{G}$ and $6-311+\mathrm{G}(\mathrm{d}, \mathrm{p})$ basis sets) on these optimized structures to obtain localized bonding data, i.e. their corresponding Lewis structure. Two different basis-sets were used in all of these calculations and their results were compared to ascertain if there were any basis-set dependent effects which may lead to erroneous results. Both basis-sets contain diffused functions which are essential for molecules with lone electron pairs and vital for anions. The 6-311+G (d, p) basis-set contain polarization functions which allow more flexibility in the atomic orbital in cases where polarization or electron cloud distortions are anticipated.

Interactions between filled NBOs (orbitals with 2 electrons localized on an atom or in a bond between atoms) and empty nonLewis NBOs (empty orbitals) were also extracted from the NBO calculations. In essence, the extent of pi-bond formation via donation of a lone electron pair of the terminal atoms, $\mathrm{F}, \mathrm{O}, \mathrm{N}$ to $\mathrm{B}$ on each of the respective molecules of $\mathrm{BF} 3, \mathrm{~B}(\mathrm{OH})_{3}$ and $\mathrm{B}\left(\mathrm{NH}_{2}\right)_{3}$ was also determined from this interaction data. Delocalization was determined by the partial filling of non-Lewis orbitals and also provided a measure of the degree of departure from the ideal Lewis structure of localized bonds. ${ }^{13}$

Population Analysis - Hirschfeld population analysis for the 3 highest occupied and 3 lowest virtual orbitals were also performed on these molecules using Moller-Plesset (MP2) theory with the $6-311+\mathrm{G}(\mathrm{d}, \mathrm{p})$ basis set to obtain the atomic charges on the atoms. Natural Population Analysis (NPA) was also calculated using the same level of theory and basis set for reference purposes. 
RESULTS AND DISCUSSION

\begin{tabular}{|c|c|c|c|c|c|c|}
\hline Molecule $\rightarrow$ & \multicolumn{2}{|c|}{$\mathrm{BF}_{3}$} & \multicolumn{2}{|c|}{$\mathrm{B}(\mathrm{OH})_{3}$} & \multicolumn{2}{|c|}{$\mathrm{B}\left(\mathrm{NH}_{2}\right)_{3}$} \\
\hline Method $\downarrow$ & B-F $(\AA)$ & $\mathrm{F}-\mathrm{B}-\mathrm{F} \angle\left({ }^{\circ}\right)$ & B-O $(\AA)$ & $\mathrm{O}-\mathrm{B}-\mathrm{O} \angle\left(^{\circ}\right)$ & B-N $(\AA)$ & $\mathbf{N}-\mathbf{B}-\mathbf{N} \angle\left(^{\circ}\right)$ \\
\hline $\begin{array}{c}\text { DFT-B3LYP } \\
6-31+G\end{array}$ & 1.35158 & 120 & 1.38279 & 120 & 1.43838 & 120 \\
\hline $\begin{array}{l}\text { DFT-B3LYP } \\
6-311+G(d, p)\end{array}$ & 1.31766 & 120 & 1.36958 & 120 & 1.43227 & 120 \\
\hline $\begin{array}{l}\text { MP2 } \\
311+G(d, p)\end{array}$ & 1.3825 & 120 & 1.37252 & 120 & 1.43879 & 120 \\
\hline
\end{tabular}

Table 1a. Optimized geometry parameters, using DFT and MP2 with the listed basis-set for $\mathrm{BF}_{3}, \mathrm{~B}(\mathrm{OH})_{3}$ and $\mathrm{B}\left(\mathrm{NH}_{2}\right)_{3}$.

\begin{tabular}{|c|c|c|c|c|c|c|}
\hline \multirow{2}{*}{$\begin{array}{c}\text { Molecule } \rightarrow \\
\text { Method } \downarrow\end{array}$} & \multicolumn{2}{|c|}{${ }_{2}$ HB-F } & \multicolumn{2}{|c|}{${ }_{2} \mathrm{HB}-\mathrm{OH}$} & \multicolumn{2}{|c|}{${ }_{2} \mathrm{HB}-\mathrm{NH}_{2}$} \\
\hline & B-F $(\AA)$ & $\mathrm{H}-\mathrm{B}-\mathrm{H} \angle\left(^{\circ}\right)$ & B-O $(\AA)$ & $\mathrm{H}-\mathrm{B}-\mathrm{H} \angle\left(^{\circ}\right)$ & B-N $(\AA)$ & $\mathrm{H}-\mathrm{B}-\mathrm{H} \angle\left(^{\circ}\right)$ \\
\hline $\begin{array}{l}\text { DFT-B3LYP } \\
6-311+G(d, p)\end{array}$ & 1.32862 & 120 & 1.36238 & 120 & 1.39124 & 114 \\
\hline
\end{tabular}

Table 1b. Optimized geometry parameters, using DFT with the listed basis-set for $\mathrm{BH}_{2}-\mathrm{F}, \mathrm{BH}_{2}-\mathrm{OH}$ and $\mathrm{BH}_{2}-\mathrm{NH}_{2}$.

\section{Geometry}

Geometry optimized bond lengths and angles are presented in Table 1a and $\mathbf{1 b}$. For the series $\mathrm{BF}, \mathrm{B}(\mathrm{OH})_{3}, \mathrm{~B}\left(\mathrm{NH}_{2}\right)_{3}$ there is little deviation from the trigonal planar structure with 120 bond angles. On the other hand, for the $\mathrm{BH}_{2}-\mathrm{F}, \mathrm{BH}_{2}-\mathrm{OH}^{\circ}$ and $\mathrm{BH}_{2}-\mathrm{NH}_{2}$ series, there is a noticeable deviation from the ideal trigonal planar structure for the amino-borane $\mathrm{BH}_{2}-\mathrm{NH}_{2} \mathrm{molecule}$

\section{$\mathrm{BF}_{3}$}

Three identical single $\mathrm{B}-\mathrm{F}$ bonds are obtained from the $\mathrm{NBO}$ calculations of this study for $\mathrm{BF}_{3}$ and this observation and the corresponding bond length are consistent with literature values. ${ }^{14,15}$ The electron occupancy of the three occupied p-orbital localized on each of the $\mathrm{F}$ atoms that are parallel, Figure 3 for the spatial orientation, to the empty p-orbital on B are also listed in this table; depletion of electron population in these p-orbitals is observed when compared to the ideal occupancy value of two electrons. Properties of the empty p-orbital on B are also listed in Table 2; delocalization of electron density into this orbital is supported by its' non-zero electron population. The source of this electron density is ascertained from the data in Table 3. For $\mathrm{BF}_{3}$, the major delocalized interactions are between the donor filled p-orbitals on the terminal $\mathrm{F}$ atoms and the empty $\mathrm{p}$-orbital on $\mathrm{B}$, listed in Table 2. The degree of a donation from each of the terminal $\mathrm{F}$ atoms is determined from the occupancy, column 2 , of Table 2 which indicates equal donation from each of the terminal $\mathrm{F}$ atoms into B. From this data it is concluded that each $\mathrm{F}$ donates roughly $1 / 10^{\text {th }}$ of an electron to $\mathrm{B}$. The work of See, who proposed a formal trivalent structure for $\mathrm{BF}_{3}{ }^{14}$ is well supported by this study and the very limited electron delocalization across the molecular framework giving rise to a very minuscule fractional pi-bond across the p-orbitals are also consistent with the ideas of Straub. ${ }^{15}$ The commonly accepted chemical notion that $\mathrm{BF}_{3}$ does not obey the octet rule is strongly supported by our results.

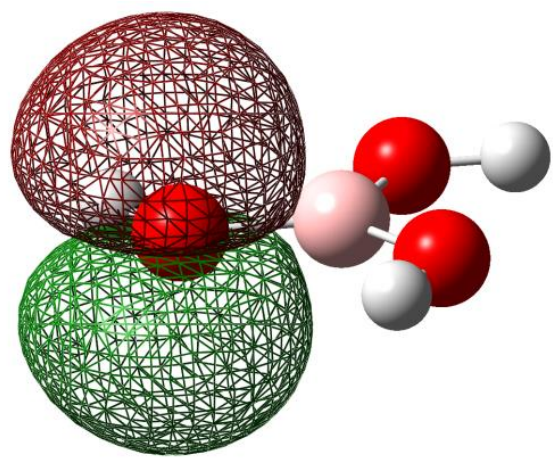

Figure 3. Representative example of the p-orbital on the terminal atom that is parallel to the empty orbital on B. Those described in Table 1 and 2. 


\begin{tabular}{|c|c|c|c|c|}
\hline $\mathbf{B F}_{3}$ & Occupancy & Nature of orbital & Atoms involved \& \% atomic orbital contribution to bond & Hybridization of F \\
\hline & 1.99825 & $\mathrm{BD}$ & $17.23 \%$ Boron and 82.77\% Fluorine & $\mathrm{sp}^{2}$ \\
$\mathbf{s p}^{2}$
\end{tabular}

\begin{tabular}{|c|c|c|c|c|}
\hline$\overline{\mathrm{B}(\mathrm{OH})_{3}}$ & Occupancy & Nature of orbital & Atoms involved \& $\%$ atomic orbital contribution to bond & Hybridization of $\mathrm{O}$ \\
\hline & $\begin{array}{l}1.99583 \\
\mathbf{1 . 9 9 6 2 7}\end{array}$ & $\mathrm{BD}$ & $\begin{array}{c}20.25 \% \text { Boron and } 79.75 \% \text { Oxygen } \\
\mathbf{2 1 . 4 4 \%} \text { Boron and } \mathbf{7 8 . 5 6 \%} \text { Oxygen }\end{array}$ & $\begin{array}{c}\mathrm{sp}^{2} \\
\mathrm{sp}^{1.4}\end{array}$ \\
\hline & $\begin{array}{l}1.99583 \\
1.99627\end{array}$ & $\mathrm{BD}$ & $\begin{array}{l}20.25 \% \text { Boron and } 79.75 \% \text { Oxygen } \\
\mathbf{2 1 . 4 4 \%} \text { Boron and } \mathbf{7 8 . 5 6 \%} \text { Oxygen }\end{array}$ & $\begin{array}{c}\mathrm{sp}^{2} \\
\mathrm{sp}^{1.4}\end{array}$ \\
\hline & $\begin{array}{l}1.99583 \\
\mathbf{1 . 9 9 6 2 7}\end{array}$ & $\mathrm{BD}$ & $\begin{array}{l}20.25 \% \text { Boron and } 79.75 \% \text { Oxygen } \\
\mathbf{2 1 . 4 4 \%} \text { Boron and } 78.56 \% \text { Oxygen }\end{array}$ & $\begin{array}{c}\mathrm{sp}^{2} \\
\mathrm{sp}^{1.4}\end{array}$ \\
\hline & $\begin{array}{l}1.85823 \\
\mathbf{1 . 8 6 1 3 7}\end{array}$ & LP & Oxygen & $\mathrm{p}$ \\
\hline & $\begin{array}{l}1.85823 \\
1.86138\end{array}$ & LP & Oxygen & $\mathrm{p}$ \\
\hline & $\begin{array}{l}1.85823 \\
\mathbf{1 . 8 6 1 3 7}\end{array}$ & LP & Oxygen & $\mathrm{p}$ \\
\hline & $\begin{array}{l}0.42410 \\
\mathbf{0 . 4 0 3 8 3}\end{array}$ & LP* & Boron & $\mathrm{p}$ \\
\hline
\end{tabular}

\begin{tabular}{|c|c|c|c|c|}
\hline $\mathbf{B}\left(\mathbf{N H}_{2}\right)_{3}$ & Occupancy & Nature of orbital & Atoms involved \& \% atomic orbital contribution to bond & Hybridization of N \\
\hline & 1.98888 & BD & $25.22 \%$ Boron and 74.78\% Nitrogen & $\mathrm{sp}^{2}$ \\
$\mathbf{s p}^{1.2}$
\end{tabular}

Table 2. Localized bonds found in the Lewis structures of $\mathrm{BF}_{3}, \mathrm{~B}(\mathrm{OH})_{3}$ and $\mathrm{B}\left(\mathrm{NH}_{2}\right)_{3}$.

Column 1 provides the name of the molecule.

Column 2 gives the occupancy of the orbital.

Column 3 describes the nature of the orbital - BD is bonding, LP is a lone pair, LP* is an empty orbital, e.g. a p-orbital.

Column 4 gives the percentage contribution of each atom's atomic orbitals to the bonding orbital (NBO).

Column 5 provides information on the hybridization of the respective atoms involved in the bond. Occupancy of any LP* orbitals reflect a deviation from the ideal Lewis formalism and indicates resonance is present in the molecule.

Entries in bold are results using the DFT-B3LYP and 6-311+G (d, p) basis-set while entries in standard font are results from 6-31+G. 


\begin{tabular}{|c|c|c|c|}
\hline Molecule & Donor & Acceptor & S. E \\
\hline $\mathrm{BF}_{3}$ & LP on Fluorine p-orbital & Boron p-orbital & 46.88 \\
\hline $\mathrm{B}(\mathrm{OH})_{3}$ & LP on Oxygen p-orbital & Boron p-orbital & $5.0(\mathrm{~F})$ \\
\hline $\mathrm{B}\left(\mathrm{NH}_{2}\right)_{3}$ & LP on Nitrogen p-orbital & Boron p-orbital & 30.34 \\
& & & $5.5(\mathrm{O})$ \\
\hline
\end{tabular}

Table 3. Interactions between Lewis orbitals (filled) and non-Lewis (empty) of the respective molecules listed in column 1. It shows the extent of a donation from filled orbitals into empty orbitals and is a measure of the delocalization of electron density. All of the p-orbitals in the table are parallel to the empty p-orbital on $\mathrm{B}$. Note the increasing degree of interaction as one goes from $\mathrm{F}, \mathrm{O}$ and $\mathrm{N}$.

Column 1 lists the names of the molecules.

Column 2 is the atom containing the filled donor orbital.

Column 3 is the atom containing the empty acceptor orbital.

Column 4 is the Stabilization Energy (S.E) from the donor-acceptor interactions in units of $\mathrm{kcal} / \mathrm{mol}$.

Column 5 is the respective electronegativity of each donor.

Entries in bold are results using the $6-311+\mathrm{G}(\mathrm{d}, \mathrm{p})$ basis-set and entries in standard font are results from 6-31+G.

\begin{tabular}{|c|c|c|}
\hline Molecule & $\begin{array}{c}\text { Atomic charge for central Boron atom } \\
\text { (Hirschfeld) }\end{array}$ & Atomic charge for central Boron atom (NPA) \\
\hline $\mathrm{BF}_{3}$ & 0.527606 & 1.57931 \\
\hline $\mathrm{B}(\mathrm{OH})_{3}$ & 0.395884 & 1.37144 \\
\hline $\mathrm{B}\left(\mathrm{NH}_{2}\right)_{3}$ & 0.265733 & 1.12118 \\
\hline
\end{tabular}

Table 4. Atomic charges were obtained from MP2 calculations with the $6-311+\mathrm{G}(\mathrm{d}, \mathrm{p})$ basis set using bot the Hirschfeld and NPA methods.

\section{$\mathbf{B}(\mathbf{O H})_{3}$}

Similar to $\mathrm{BF}_{3}$, formally three single $\mathrm{B}-\mathrm{O}$ bonds from $\mathrm{B}$ to the terminal $\mathrm{OH}$ groups are present in $\mathrm{B}(\mathrm{OH})_{3}$. The calculated $\mathrm{B}-\mathrm{O}$ bond length is comparable top literature value; ${ }^{11}$ although the hybridization of $\mathrm{O}$ in $-\mathrm{OH}$ is expected to be sp ${ }^{3}$, there is rehybridization of these orbitals to generate a p-orbital for overlap with the empty p-orbital on B, column 5 of Table 2 . These three occupied donor $\mathrm{p}$-orbitals on the $\mathrm{O}$ of the terminal $-\mathrm{OH}$ groups, parallel to the $\mathrm{p}$-orbital on $\mathrm{B}$, are also listed in Table 2 . The electron occupancy of these p-orbitals is more diminished with a more noticeable increase in the electron occupancy of $\mathrm{B}$ for this molecule when compared to $\mathrm{BF}_{3}$. Even though this electron delocalization has increased, it nonetheless amounts to no more than just $1 / 7^{\text {th }}$ of an electron donation by each $\mathrm{O}$ in $\mathrm{B}(\mathrm{OH})_{3}$ when compared to $1 / 10^{\text {th }}$ in $\mathrm{BF}_{3}$; thus, delocalization may have increased with a decrease in $\mathrm{EN}$ but is not sufficient to change the established idea that $\mathrm{B}(\mathrm{OH})_{3}$ doesn't obey the octet rule.

\section{$\mathbf{B}\left(\mathrm{NH}_{2}\right)_{3}$}

Analogous to the above two molecules, formally three single $\mathrm{B}-\mathrm{N}$ bonds are found in this molecule. The $\mathrm{B}-\mathrm{N}$ bond length is consistent with literature value. ${ }^{16}$ Most notable observation is the even further diminished electron occupancy of the $\mathrm{p}$-orbitals on the $\mathrm{N}$ of the respective terminal $-\mathrm{NH}_{2}$ groups and the greatest electron occupancy of the empty p-orbital of $\mathrm{B}$ in this group of molecules. Once again, the hybridization on the $\mathrm{N}$ of $-\mathrm{NH}_{2}$ is expected to be $\mathrm{sp}^{3}$ but it is seen that there is also substantial rehybridization to produce a p-orbital for overlap with that on B. These occupied p-orbitals of $\mathrm{N}$ are also listed in Table 1. This increased delocalization though amounts to no more than roughly just $1 / 6^{\text {th }}$ of an electron donation from each N's occupied $\mathrm{p}$ orbital into the empty p-orbital of $\mathrm{B}$. Although there is a trend of increasing electron donation from $\mathrm{p}$-orbitals of $\mathrm{F}$, $\mathrm{O}$, and $\mathrm{N}$ respectively as a function of decreasing $\mathrm{EN}$, these contributions are insufficient to create a pi-bond in these boron compounds. Thus the octet rule does not apply to the bonding in this molecule.

The trend of increasing electron delocalization as a function of decreasing EN of the terminal atoms is also evident in the trend of atomic charges on B across this series of molecules, see Table 4. In order to independently verify the results of the NBO analysis above, atomic charges calculated using Hirschfeld population analysis on the three highest energy filled molecular orbitals and three higher energy virtual orbitals were performed. A trend of decreasing positive atomic charge on B is observed when the terminal atom changes from $\mathrm{F}$ to $\mathrm{O}$ to $\mathrm{N}$. This observation is consistent with the NBO results of increasing electron density on $\mathrm{B}$ for this set of molecules. This trend is also seen in the NPA results which are also included in Table 4 for reference purposes. Note that in general, different population analysis schemes give different numerical values of atomic charges and cautions should be exercised when comparing absolute values. 
An alternative to $\mathrm{NBO}$ and Population Analysis in ascertaining the degree of electron donation from the terminal $\mathrm{F}, \mathrm{O}$ and $\mathrm{N}$ to $\mathrm{B}$ and thus potential pi-bonding is to study the corresponding relative bond lengths of the $\mathrm{BH}_{2}-\mathrm{F}, \mathrm{BH}_{2}-\mathrm{OH}$ and $\mathrm{BH}_{2}-\mathrm{NH}_{2}$ series. The relative bond lengths is defined as the ratio of the length of $\mathrm{B}-\mathrm{X}$ bond in $\mathrm{BX}_{3}$ to the length of $\mathrm{B}-\mathrm{X}$ bond in $\mathrm{BH}_{2} \mathrm{X}$, i.e. $\left(\mathrm{B}-\mathrm{X}\right.$ bond in $\left.\mathrm{BX}_{3}\right) /\left(\mathrm{B}-\mathrm{X}\right.$ bond in $\left.\mathrm{BH}_{2} \mathrm{X}\right)$. From the data in Table 1a and $\mathbf{b}$, it is observed that there are only small changes in these relative bond lengths across the series except for $\mathrm{BH}_{2}-\mathrm{NH}_{2}$. Since $\mathrm{B}$ and $\mathrm{N}$ are neighbors in the periodic table it is expected that they would have better orbital overlap and this observation is consistent with literature results. ${ }^{9}$ The calculate $\mathrm{BH}_{2}-\mathrm{NH}_{2}$ from this study is very close to that of a double bond and warrants further study. A full NBO and Population Analysis on the $\mathrm{BH}_{2}-\mathrm{F}, \mathrm{BH}_{2}-\mathrm{OH}$ and $\mathrm{BH}_{2}-\mathrm{NH}_{2}$ series is underway.

Our initial hypothesis that changes in EN may lead to pi-bond formation is incorrect as a change in $\mathrm{EN}$ from 4 for $\mathrm{F}$ to 3 for $\mathrm{O}$ resulted in minuscule change in electron delocalization from the filled p-electrons of the terminal atoms onto the empty p-orbital of $\mathrm{B}$. Undoubtedly there is increased stabilization when the terminal atom changes from $\mathrm{F}, \mathrm{O}$ and $\mathrm{N}$ as seen in the data in Table 3 but this is not sufficient to create a pi-bond.

\section{$-\mathrm{O}-\mathrm{B}(\mathrm{OH})_{2}$}

Some insight was gained through this study even though our initial hypothesis proved to be incorrect. The systematic increase in electron delocalization as EN decreased, coupled with the idea that EN is inherently related to the energy of an atom's orbital energy suggested that more efficient electron delocalization may be achieved if the two interacting orbitals have better energy match. ${ }^{17}$ Upon inspection of the relative energy levels of the p-orbital on $\mathrm{B}$ to those on $\mathrm{F}, \mathrm{O}$ and $\mathrm{N}$ respectively (i.e. orbital energy as a function of nuclear charge), Figure 4, a progressively better energy match of $\mathrm{F}, \mathrm{O}$ and $\mathrm{N}$ respectively to that of $\mathrm{B}^{18}$ is observed.

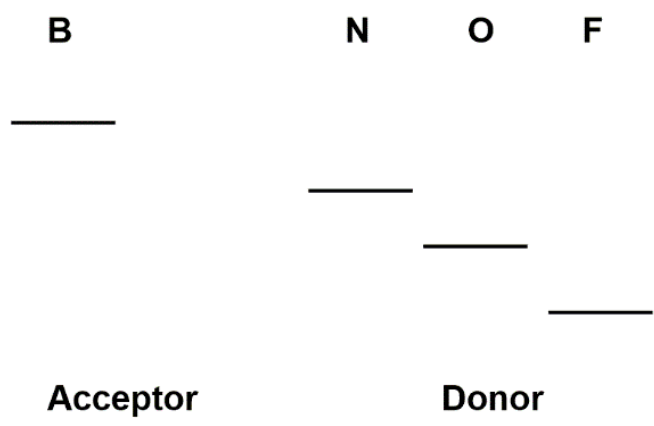

Figure 4. Relative energies of the p-orbitals from B, N, O and F.

Thus, stronger electron delocalization may in principle occur for a chemical species that contained a terminal atom with a donor orbital that is better matched in energy to the acceptor orbital of the central atom.

In order to test this new hypothesis, calculations were performed on the borate ion, ${ }^{-} \mathrm{O}-\mathrm{B}(\mathrm{OH})_{2}$, i.e. the anion of $\mathrm{B}(\mathrm{OH})_{3}$. The anion is expected to have a pair of higher energy electrons (i.e. the negative charge residing on the oxygen) compared to the lone pair on a neutral $-\mathrm{OH}$ or $-\mathrm{NH}_{2}$ terminal group, and this higher energy pair may thus interact more efficiently with the empty $\mathrm{p}$ orbital of B. Optimized geometric parameters of this anion is given in Figure 5. Results from the NBO calculations on the borate ion are given in Table 5 .

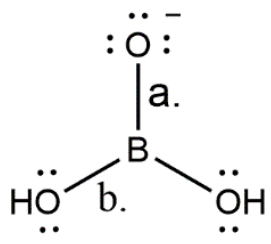

Figure 5. Optimized geometry of the borate ion using DFT-B3LYP $6-311+\mathrm{G}(\mathrm{d}, \mathrm{p}) \cdot \mathrm{a}=1.27797 \AA_{\AA}$ and $\mathrm{b}=1.46486 \AA_{\AA}$. The $\mathrm{HO}^{-} \mathrm{B}_{-} \mathrm{O}^{-}$bond angle is $124^{\circ}$ and

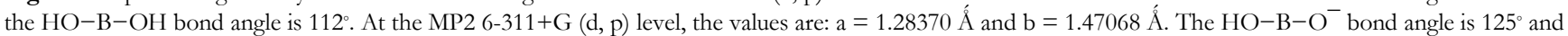
the $\mathrm{HO}-\mathrm{B}-\mathrm{OH}$ bond angle is $110^{\circ}$. 


\begin{tabular}{|c|c|c|c|c|}
\hline$-\mathrm{O}-\mathrm{B}(\mathrm{OH})_{2}$ & Occupancy & $\begin{array}{c}\text { Nature of } \\
\text { orbital }\end{array}$ & $\begin{array}{l}\text { Atoms involved } \& \% \text { atomic orbital } \\
\text { contribution to bond }\end{array}$ & $\begin{array}{l}\text { Hybridization } \\
\text { of Oxygen }\end{array}$ \\
\hline & $\begin{array}{l}1.91772 \\
1.92325\end{array}$ & $\mathrm{BD}$ & $\begin{array}{l}14.45 \% \text { Boron and } 85.55 \% \text { Oxygen } \\
\mathbf{1 5 . 5 1 \%} \text { Boron and } \mathbf{8 4 . 4 9 \%} \text { Oxygen }\end{array}$ & $\begin{array}{c}\mathrm{sp} \\
\mathrm{sp}^{1.8}\end{array}$ \\
\hline & $\begin{array}{l}1.91772 \\
1.92325 \\
\end{array}$ & $\mathrm{BD}$ & $\begin{array}{c}14.45 \% \text { Boron and } 85.55 \% \text { Oxygen } \\
\mathbf{1 5 . 5 1 \%} \text { Boron and } \mathbf{8 4 . 4 9 \%} \text { Oxygen }\end{array}$ & $\mathrm{sp}^{1.8}$ \\
\hline & $\begin{array}{l}1.99283 \\
1.99385\end{array}$ & $\mathrm{BD}$ & $\begin{array}{l}22.32 \% \text { Boron and } 77.68 \% \text { Oxygen } \\
\mathbf{2 3 . 4 1 \%} \text { Boron and } \mathbf{7 6 . 5 9 \%} \text { Oxygen }\end{array}$ & $\mathrm{sp}$ \\
\hline & $\begin{array}{l}1.99884 \\
1.99882\end{array}$ & $\mathrm{BD}$ & $\begin{array}{l}17.83 \% \text { Boron and } 82.17 \% \text { Oxygen } \\
\mathbf{1 6 . 8 4 \%} \text { Boron and } \mathbf{8 3 . 1 6 \%} \text { Oxygen }\end{array}$ & $\mathrm{p}^{*}$ \\
\hline
\end{tabular}

Table 5. Localized bonds found in the Lewis structure of the ${ }^{-} \mathrm{O}-\mathrm{B}(\mathrm{OH})_{2}$ anion.

*a p-orbital is used on B to form the BD orbital for this entry.

The description of each column is identical to that in Table 2.

In this case, four bonds are clearly shown in the NBO analysis. The first two entries in Table $\mathbf{5}$ are the single bonds from B to the $\mathrm{OH}$ groups while the third and fourth entries are for the sigma single bond between $\mathrm{B}$ and $\mathrm{O}$ followed by the pi-bond ( $\mathrm{p}-\mathrm{p}$ interaction) between the same two atoms. Figure 6 provides a visualization of this pi-bond between the terminal $\mathrm{O}$ and the central B. Further support for the presence of the pi-bond is seen in the unequal $\mathrm{B}-\mathrm{O}^{-}$and $\mathrm{B}-\mathrm{OH}$ bond lengths with the shorter one for the double bond. Previously reported values by Straub are consistent with our results. The magnitude and unequal nature of the $\mathrm{HO}-\mathrm{B}-\mathrm{O}^{-}$and the $\mathrm{HO}-\mathrm{B}-\mathrm{OH}$ bond angles are also consistent and very similar to carbonic acid $\mathrm{HO}-\mathrm{CO}-\mathrm{OH}{ }^{19}$ which has is identical structurally to the borate ion.

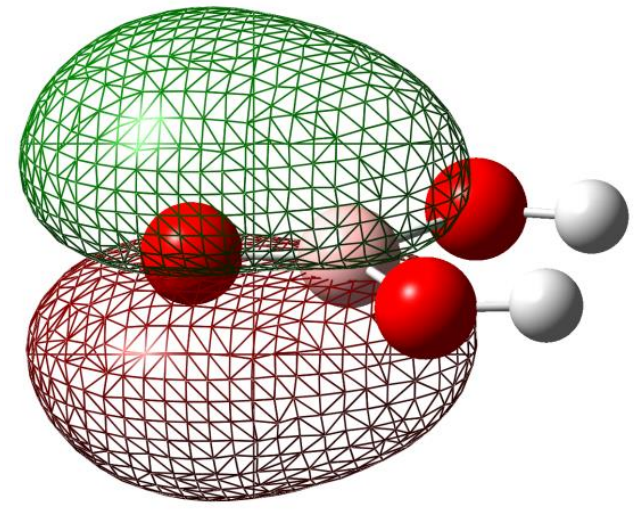

Figure 6. Visualization of the localized pi-bond orbital in the ${ }^{-} \mathrm{O}-\mathrm{B}(\mathrm{OH})_{2}$ borate anion.

\section{CONCLUSION}

This study sought to understand the effects of EN of the terminal atoms on the formation of delocalized pi-bonding to the central $\mathrm{B}$ atom in the series $\mathrm{BF}_{3}, \mathrm{~B}(\mathrm{OH})_{3}$ and $\mathrm{B}\left(\mathrm{NH}_{2}\right)_{3}$. The starting hypothesis that EN played a central role in this delocalization and potential pi-bond formation was shown to be false. Even with fairly large changes in EN, the degree of electron donation from the terminal atoms although increasing was minimal. Despite this, some insight into another factor that influenced pibonding in boron compounds was obtained and this insight revealed that improved energy match between the filled donor orbitals of the terminal atoms and the empty p-orbital of boron do lead to pi-bond formation.

\section{ACKNOWLEDGEMENT}

The authors thank the Metropolitan Community College (MCC) Foundation for a mini-grant to support this work; the MCC IT department for their technical assistance and the Dean of Math \& Natural Sciences, Mike Flesch. We also thank Dr. J.R. Schmidt of WebMO for his assistance and PerkinElmer Informatics/CambridgeSoft for the use of their ChemDraw program on a 14-day trial. 


\section{REFERENCES}

1. Gillespie, R.J., Robinson, E.A. (2007) Gilbert N. Lewis and the Chemical Bond: The Electron Pair and the Octet Rule from 1916 to the Present Day, J Comp Chem Special Issue 28, Issue 1, pp 87-97.

2. Kotz, J.C., Treichel, P.M., Townsend, J.R., and Treichel, D.A. (2015) Chemistry \& Chemical Reactivity 9th ed., pp 286-302, Cengage Learning, Stamford, Connecticut.

3. Whitten, K.W., Davis, R.E., Peck, M.L., and Stanley, G.G. (2010) Chemistry 9th ed., pp 262-270, Brooks/Cole, Belmont, California.

4. Bessac, F., Frenking, G. (2003) Why is BCl3 a Stronger Lewis Acid with Respect to Strong Bases than BF3? Inorg Chem 42, pp 7990-7994.

5. House, J.E., House, K.A. (2016) Descriptive Inorganic Chemistry 3rd ed., pp 30, Elsevier Inc., San Diego, California.

6. Franca, G.A., Diez, R.P. (2009) The Lewis Acidity of Boron Trihalides Revisited, J. Argent Chem Soc 97, pp 119-126.

7. Heller, G. (1993) Gmelin Handbook of Inorganic and Organometallic Chemistry - Boron Compounds 8th ed. 4th Supplement Vol. 2, Springer Verlag, Berlin Heidelberg.

8. Meller, A. (1991) Gmelin Handbook of Inorganic and Organometallic Chemistry - Boron Compounds 8th ed., 4th Supplement Vol. 3a, Springer Verlag, Berlin Heidelberg.

9. Kormos, B.L., Cramer, C.J. (2003) Pi Bonding and Negative Hyperconjugation in Mono-, Di-, and Triaminoborane, -alane, gallane and -indane Inorg. Chem 42, pp 6691-6700.

10. House, J.E., House, K.A. (2016) Descriptive Inorganic Chemistry 3rd ed., pp 141, Elsevier Inc., San Diego, California.

11. Carey, F.A., Sundberg, R.J. (2007) Advanced Organic Chemistry 5th ed., pp 8, Springer Science+Business Media, LLC, New York.

12. Gaussian 09, Revision D.01, M. J. Frisch, G. W. Trucks, H. B. Schlegel, G. E. Scuseria, M. A. Robb, J. R. Cheeseman, G. Scalmani, V. Barone, B. Mennucci, G. A. Petersson, H. Nakatsuji, M. Caricato, X. Li, H. P. Hratchian, A. F. Izmaylov, J. Bloino, G. Zheng, J. L. Sonnenberg, M. Hada, M. Ehara, K. Toyota, R. Fukuda, J. Hasegawa, M. Ishida, T. Nakajima, Y. Honda, O. Kitao, H. Nakai, T. Vreven, J. A. Montgomery, Jr., J. E. Peralta, F. Ogliaro, M. Bearpark, J. J. Heyd, E. Brothers, K. N. Kudin, V. N. Staroverov, T. Keith, R. Kobayashi, J. Normand, K. Raghavachari, A. Rendell, J. C. Burant, S. S. Iyengar, J. Tomasi, M. Cossi, N. Rega, J. M. Millam, M. Klene, J. E. Knox, J. B. Cross, V. Bakken, C. Adamo, J. Jaramillo, R. Gomperts, R. E. Stratmann, O. Yazyev, A. J. Austin, R. Cammi, C. Pomelli, J. W. Ochterski, R. L. Martin, K. Morokuma, V. G. Zakrzewski, G. A. Voth, P. Salvador, J. J. Dannenberg, S. Dapprich, A. D. Daniels, O. Farkas, J. B. Foresman, J. V. Ortiz, J. Cioslowski, and D. J. Fox, Gaussian, Inc., Wallingford CT, 2013.

13. Natural Bond Orbital Analysis - Tutorial Example - NBO, http:// nbo6.chem.wisc.edu/tutorial.html (accessed January 2016)

14. See, R.F. (2009) Which Method of Assigning Bond Orders in Lewis Structures Best Reflects Experimental Data? An Analysis of the Octet Rule and Formal Charge Systems for Period 2 and 3 Nonmetallic Compounds, J Chem Ed 86, pp 1241-1247.

15. Straub, D.K. (1995) J Chem Ed 72, pp 494-497.

16. Berski, S., Latajka, Z., Gordon, A.J. (2011) On the multiple B-N bonding in boron compounds using the topological analysis of electron locatization function (ELF), New J. Chem 35, pp 89-96.

17. Coulson, C.A. (1961) Valence 2nd ed., pp 74, Oxford University Press, London.

18. Bader, R.F.W. (2010) An Introduction to the Electronic Structure of Atoms and Molecules, http:/ / www.chemistry.momaster.ca/ esam/Chapter_5/section_2.html (accessed January 2016)

19. Reedy, S.K., Kulkarni, C.H., Balasubramanian S. (2011) Theoretical investigations of candidate crystal structures for $\beta$ carbonic acid, J. Chem. Phys 134, pp 124511-124519.

\section{ABOUT THE STUDENT AUTHOR}

Xavier Shiu, an outstanding student and a Metropolitan Community College Student Ambassador, is currently at the University of Nebraska - Lincoln completing his Bachelor's degree in Mechanical Engineering.

\section{PRESS SUMMARY}

Understanding the bonding in the element boron (B) has proven to be a challenge. This paper investigates the effects of electronegativity $(\mathrm{EN})$ on the bonding in B. Different atoms, namely fluorine $(\mathrm{F})$, oxygen $(\mathrm{O})$ and nitrogen $(\mathrm{N})$ respectively are bonded to boron and the nature of the B-F, B-O and B-N bonds are studied theoretical. Since the EN of F, O and N are different, this study may shed light on the effects of changing electronegativity on the bond between $\mathrm{B}$ and these other elements. 


\title{
What Angle Will You Take? Patterns of Perspective-Taking in a Body- Based Task
}

\author{
Tegan Garon \\ Department of Education, School of Education and Social Services, University of Vermont, Burlington, VT \\ Student: teags3@yahoo.com* \\ Mentor: carmen.smith@uvm.edu*
}

\begin{abstract}
When people conceptualize abstract ideas, different perspectives can help them to make connections and develop their reasoning. In this study, three third grade students who engaged in a body-based angle task, using the Kinect for Windows, were analyzed. Descriptions of their interviews are presented, including a detailed analysis of their patterns of perspective-taking and factors that might have been conducive to their learning. It was observed that students typically adopted a body-based perspective before transitioning to other perspectives. Further, the design of the task and interviewer comments were important factors that prompted students to take different perspectives. The implications for perspectives and their impacts on learning are discussed.
\end{abstract}

\section{KEYWORDS}

Embodied Cognition; Angles; Mathematics Education; Perspectives; Movement

\section{INTRODUCTION}

The act of learning mathematics necessitates a particular type of thinking, one in which a conceptual approach has been shown to be more useful than memorizing formulas and procedures. ${ }^{1}$ In order for students to develop conceptual understanding, mathematics instruction ought to scaffold students to make connections among concrete concepts, eventually resulting in the formation of abstract ideas.

Studies have shown that embodying concepts has become a new way for students to make meaning, since it promotes linkage between reasoning and physical movements, thus allowing students to connect seemingly unrelated ideas. ${ }^{2,3}$ Body-based activities can do more than allow students to relate ideas; they can actually help students to learn, particularly in mathematics. ${ }^{3,4}$ Learning occurs when a bridge is built between physical movements of a body and concepts stemming from cognition and reasoning. ${ }^{2}$ Body-based activities have been important in students' mathematical learning processes, 5,6 although a definitive relationship between embodiment and learning gains is still being explored by researchers and scholars. Nonetheless, body-based activities that seem conducive to learning are those that allow students to assume a first-person perspective while engaging in task..$^{7,8}$

In this paper, we seek to investigate the conditions of body-based activities that support learning, including initially prompting students to take on the first-person perspective, and then providing access for them to advance to other perspectives. We will also consider the patterns of perspective-taking that three third grade students demonstrated while completing a body-based angle task using the Kinect for Windows.

\section{BACKGROUND}

Embodied cognition is a formal way of classifying how cognition and reasoning interact with the physical world around us. ${ }^{9}$ "Embodiment" is essentially a term that reflects how perceptual and motor skills play a prominent role in the formation of concepts. Reasoning and physical action are not separate activities; they are deeply integrated. ${ }^{10}$ Even thinking that requires the use of abstract concepts stems from embodied interactions with a physical environment. ${ }^{2}$ In general, body-based tasks serve as a means by which abstract mathematical ideas can be grounded in a form that is easily comprehensible and accessible for the learner. ${ }^{3}$ As a result, when students engage in body-based tasks, their understanding of abstract ideas can become more concrete, since their thinking is constructed from, as well as grounded in, physical actions. ${ }^{3}$

\section{Reasoning and Physical Interactions}

The assumption that reasoning and physical tasks are closely tied is not trivial. One might consider how it is possible for reasoning to "grow out of a cognitive task". ${ }^{10}$ Remarkably, several neural systems in the brain, those primarily responsible for perceptions, movement, and object manipulation, are the same neural systems that are utilized by the brain to achieve conceptualization and reasoning. ${ }^{10}$ Perception and reasoning always coincide with, and consequently, are structured by, a physical 
body's engagement with an environment. ${ }^{9}$ Sensory inputs that are acquired through physical experiences can be united into a cohesive entity that reflects how we manipulate and interact with the world around us.

The relationship between reasoning and embodiment is illustrated in a plausible, real-world example that many car owners, or car passengers for that matter, have surely experienced at one time or another. Michael Anderson presents the frustration of dropping a lifesaver (or similar bite-sized candy) in between the seats of a car and then realizing that there are no means by which to retrieve the lifesaver, other than using a stray paperclip. The paperclip will need to be manipulated into various positions in order to find the optimal shape that will hook the lifesaver, so through this trial-and-error experience, cognitive skills are forced to collaborate with physical capabilities. While transforming the paperclip into different positions, our mind must conceive how any given shape will be able to successfully hook the lifesaver. ${ }^{9}$ Embodied cognition requires cooperation between representation and physical movement, which exemplifies the interrelatedness between the two.

Whenever we engage in a physical task, our brains acquire a plethora of sensory inputs, stimulating the brain's perceptions that were activated during the task. The brain is able to later recall its cognitive state during the physical task; the inputs that were acquired have now become a representation of the physical activity. Therefore, when required to recall conceptual knowledge, people tend to draw upon those stored representations of the physical tasks. ${ }^{11}$ Research has shown that the greater number of sensory inputs triggered during a physical task will yield higher efficiency storage of schema since representations from the task can be recalled more easily. ${ }^{4} \mathrm{~A}$ strong ability to recall information often leads to high performance on assessment measures, which supports the position that body-based activities could result in learning gains.

In a study involving participants counting change with and without using their fingers, it was demonstrated how humans utilize cognitive resources to the best of their abilities, often relying on the assistance of physical actions. In the study, participants who were able to count by pointing to an image depicting a set of coins or counting with their fingers were more accurate than participants who were required to count the change without the assistance of their hands or fingers. ${ }^{12}$ Being able to call upon physical actions to help conceptualize ideas can result in the ability to solve larger and more complex problems. Reasoning is essentially a condensed version of knowledge that is acquired through interactions with the environment. ${ }^{1}$

In terms of embodied mathematics, a study was conducted in which students in grades 4-6 were tasked with turning a screen green by moving their hands to different positions, unaware that the screen only turned green when their hands were at a certain height above the desk, relative to each other. Eventually, students worked through the task with a Cartesian plane on the screen and continued utilizing the motion of their hands to determine how units on the plane were changing. ${ }^{13}$ In another study, participants were asked to justify mathematical tasks, such as a triangle side length conjecture, after completing various physical actions that were intended to promote either "character" (first-person) or "observer" (third-person) viewpoints. The results showed that physical actions enhanced mathematical justification, thus suggesting the linkage between embodiment and mental reasoning. ${ }^{14}$ Due to the close relationship between physical actions and cognition, we pose that body-based tasks help to shape the way we learn abstract mathematical concepts.

Perspectives During Body-Based Tasks

Body-based tasks are related to the ability for people to take on multiple perspectives during their interactions with a physical environment. ${ }^{15}$ Edith Ackermann terms the multiple perspectives that can be assumed during a body-based task as "diving in" and "stepping out". "Diving in" is referred to as completely immersing oneself in the task by "becoming one" with it, whereas "stepping out" involves reconsideration of the task from an outsider's perspective. ${ }^{5}$ Furthermore, learning can really only take place if we embrace multiple perspectives throughout the experience; one perspective is simply not enough. ${ }^{5}$ Ultimately, a balance between perspectives is ideal, since we need to immerse ourselves in the task in order for development and growth to take place, yet we must also become detached, at times, so that we can relate our thoughts to the physical task, resulting in reflection. ${ }^{5}$

Additional research has proposed that perceiving oneself as a part of the actual task may lead to greater success in solving the task. In one study, the use of perspectives was explored in a scientific setting as students engaged in a simulation called MEteor. The simulation consisted of a 30-foot by 10-foot interactive floor and prompted students to investigate Newton and Kepler's laws while viewing how their movements were affecting a graph on a screen. ${ }^{16}$ Students embodied a first-person perspective by seeing how their physical movements directly impacted the graph in front of them.

It is especially beneficial when problem solvers adopt more than one perspective. ${ }^{8}$ The multiple perspectives are described as "frames of reference", which can be generally categorized as either egocentric or allocentric. An egocentric perspective occurs when the objects/environment are in relation to one's self, resulting in short-term retention of knowledge, whereas an allocentric perspective occurs when the body is not the point of reference, and often leads to long-term retention. ${ }^{17}$ 
The multiple perspectives during a body-based task have also been termed as "being it", in which oneself is experienced as the mathematical object, and "watching it", in which a mathematical object is perceived to be separate from oneself. ${ }^{6}$ The ability to take on the first-person perspective is critical in mathematics since it leads to the formation of connections that cannot be made when a participant is exclusively an observer. ${ }^{6}$ Susan Gerofsky conducted a study in which students' and teachers' bodies were observed while they described a graph about an object in motion. Some of the participants took on the first-person perspective by demonstrating whole body movements as if they were the graphs themselves. These participants who "became the graph" were more likely than the other participants to approach their verbal explanations with multiple metaphors, thus suggesting that more than one entry point was used to make sense of the conceptual ideas of the graphs. ${ }^{7}$

Although a first-person perspective is optimal for immediate learning, a third-person perspective may yield greater long-term learning gains. ${ }^{17}$ When information acquired during a body-based task must be encoded, the body will most likely not be in the same context as it was during the task, which is why a third-person perspective becomes necessary for retrieval of stored information. ${ }^{17}$ Even when the third-person perspective has allowed for adequate retrieval of information, the first-person perspective becomes, again, necessary, so that people can continue to make sense of the task. ${ }^{17}$ Consequently, alternating between perspectives is the most useful in terms of storage, encoding, and retrieval of information.

\section{The Angle Concept and Body-Based Angle Task, (The Study)}

The concept of an angle is often represented by various definitions and illustrations throughout textbooks; the teaching of angles is not always consistent across schools. ${ }^{18}$ For example, younger students learn about angles as being fractions of circles whose measure is converted into fractions of 360 degrees. ${ }^{19}$ They try to eventually reach a notion of a "standard angle concept", which is that two lines meet at a point. ${ }^{18}$ Other students learn angles as being inclinations of two lines in a plane. ${ }^{20}$ The main issue is that angles are typically introduced in a procedural sense, lacking the emphasis that they are units of measure in degrees. ${ }^{19}$

There are also numerous misconceptions that students perceive about angles. In one study, less than $10 \%$ of fourth-grade students surveyed mentioned the "turning" feature when asked to describe angles, meaning that angles can be rotated in infinite orientations and their degree measure stays the same. ${ }^{21}$ Students may also believe that one of the rays of an angle must be horizontal, due to the limited examples they have been exposed to in textbooks. It is a common misconception that the size of an angle depends on upon the length of the line segments or the distance between the endpoints. Students' confusion could be alleviated if they were able to identify aspects of the angle concept within different representations, such as gestural, graphical, and verbal. $^{22}$

In this study, we analyzed three third grade students as they completed a body-based angle task. The task required students to physically form angles with their arms and then use their actions to create conjectures about angle properties. Since the concept of an angle is such a multifaceted entity, ${ }^{18}$ the task was designed to be particularly conducive to adapting multiple perspectives. Students might take on the first-person perspective by thinking of themselves as the angle (termed "body-based"), or they might take a third-person perspective, conceptualizing angles as abstract figures that are separate from their bodies (termed "anglebased"). Furthermore, as students translated between and coordinated the body-based and angle-based perspectives, they could also take on both perspectives simultaneously (termed "dual").

We closely examined the language and actions of three students during their engagement with the body-based task to explore the following research questions: (1) What are possible patterns of perspective-taking during this body-based task? (2) What factors can affect students' abilities to take on different perspectives?

\section{METHOD}

\section{Participants}

This work was part of a larger study that included 32 third- and fourth-grade male and female students from four multi-age classes. Students ranged in age from eight to ten years. Each student took part in a 15-20 minute, structured, one-on-one interview with a researcher, ${ }^{23}$ in which they completed a body-based task about angle concepts. No compensation was exchanged for participation in the study, and participants were permitted to withdraw at any time. All interviews were videotaped, in an effort to relieve interviewers' responsibility of writing notes during interactions with participants. This project received approval from the Institutional Review Board.

Since this was an exploratory case study, ${ }^{24}$ we sought to find new patterns in the data. Stratified purpose sampling was used ${ }^{25}$ to select individual students' cases to investigate in depth. Using recorded video, transcripts, and interview notes, we examined the three students in detail, tracking their patterns of perspective-taking. The three students were selected due to their assessment scores on a pre- and post-test, in which one student earned high scores on both assessments, another student earned low scores on both assessments, and a third student earned a low score on the pre-test and a high score on the post-test. 


\section{Materials}

Each interview lasted approximately 40 minutes, which included 15-20 minutes for participants to work on the body-based angle task, and about 10 minutes each for the administration of a pre- and post-test. A Kinect sensor bar tracked participants' physical movements, so they stood facing a large screen that projected the Kinect program. The sensor bar located and used participants' base of their necks to form the vertex of an angle, and then their arms became the rays of the angle. Participants faced the Kinect sensor bar while an interviewer was positioned nearby. A second interviewer manipulated the various levels of the Kinect program on the Windows laptop.

\section{Procedure}

The body-based task was designed to support students' gradual development of an angle concept and to correct common misconceptions. For this reasoning, it was important to scaffold students' learning by first allowing them to explore the concept of an angle without the use of any tools or additional information, other than the notion that there was an angle on the screen. As students progressed through the activity, the lengths of the rays of the angle were increased, and then students were asked to explore how the size of an angle related to specific degree measures. Since the angle oriented itself to students' arms, students could also visualize how angles of the same measure could have different orientations. Furthermore, since the screen was constantly changing colors based upon the movements of students' arms, students were able to investigate what is meant by the size of an angle.
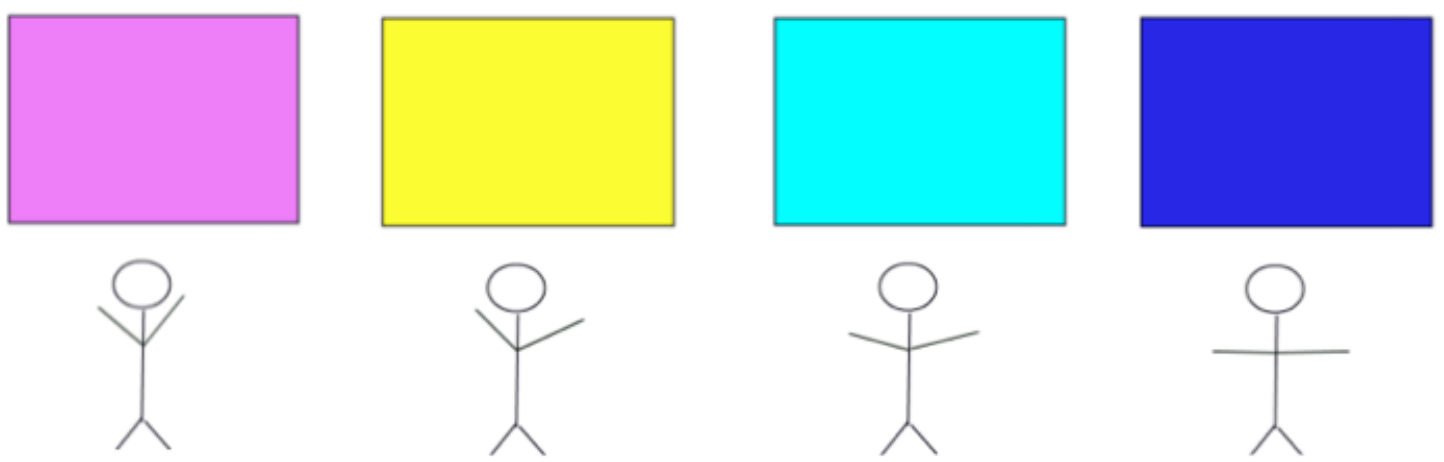

Figure 1. Representation of the four different types of angles in the body-based task with the corresponding colors of the screen.

At the start of the task, participants were instructed to extend their arms out to the side of their bodies, since the sensor bar could not track any movements if participants' arms were in front of or behind their bodies. Participants were informed that the screen in front of them would change colors, and they were asked to pose "rules" as to what made the screen each color. Unbeknownst to the participants, initially, were the conditions that changed the color of the screen: an acute angle changed the screen to pink, a right angle changed the screen to yellow, an obtuse angle changed the screen to light blue, and a straight angle changed the screen to dark blue (see Figure 1). The Kinect program was designed so that the screen changed colors for angles that were within an absolute value of three degrees from the actual measure. For example, even though a right angle, 90 degrees, caused the screen to be yellow, an angle measuring 87 to 93 degrees, inclusive, still activated the screen to be yellow. A similar pattern followed for the remaining three colors, although this information was not revealed to participants.

During the first part of the interview, participants were asked to describe what their arms were doing to make the screen different colors. The prompts were intended to be open-ended, so interviewer responses did not indicate whether participants' answers were correct or not. After participants posed conjectures as to how to make the screen each color, they were informed that a "tool" was going to be added to the screen: arrows. The vertex of the arrow matched to the base of participants' neck, and then the rays of the arrows tracked the movement of participants' arms, accordingly. For this portion of the task, participants were again asked to pose conjectures as to how to make the screen each of the four colors. If they only responded in a way that referenced the position of their arms, then the interviewer provided a follow-up question that prompted them to refer to the arrows on the screen.

In the third part of the task, a protractor was added to the screen, in which it automatically oriented itself with one of the rays of the angle and used the other ray to measure the angle, thus projecting a numerical value on the screen for participants to see. In 
addition to its projection of any numerical value from 0 to 180 degrees, inclusive, the protractor was labeled with benchmark numbers of $0,45,90,135$, and 180 degrees. At this time, participants were given approximately $30-45$ seconds to experiment freely with the protractor while the interviewer left the room. Upon the interviewer's return, participants were asked whether they had figured anything out regarding how the protractor worked. Then, the interviewer proceeded to probe participants to form conjectures about how to make the screen each color, referencing the protractor in their responses, if possible.

For the final part of the task, the protractor remained on the screen and the length of the arrows was increased dramatically. Once it had been established with participants that the length of the arrows was different from the second and third parts of the task, participants were asked to form conjectures, one last time, about how to make the screen each color. The interviewer did not follow up with reinforcing questions and essentially accepted whatever response participants offered.

\section{Design and Coding}

Measures. Various forms of data were collected from each interview, including a pre- and post-test that was administered on an $\mathrm{iPad}$ as well as video recordings of the interview. The pre- and post-tests were completely identical and required students to sketch angles that were different from each other, sketch angles that were larger and smaller than given angles, sketch angles of designated measures, and estimate the measure of angles. Eleven total questions were scored, so participants could earn a whole number score, ranging from 0 to 11 on each of the tests. A 14-question spatial reasoning task was also included in both assessments, although that section of the assessment was excluded from the analysis of this study. The pre- and post-tests are described in another study. ${ }^{26}$

Coding. While implementing stratified purposeful sampling ${ }^{25}$ in this study, we identified potential variation in the data between different types of students. All of the interviews were digitally recorded and transcribed so that student responses and interview prompts could be analyzed. We examined three students in depth. We present descriptions of the interviews in addition to an analysis of students' perspective-taking. These students were chosen based on their assessment scores: Jace performed well on both the pre- and post-test, Abdul received low scores on both the pre- and post-test, and Tony received a low pre-test score, but then improved greatly to earn a high post-test score.

Coding data is an act of stringing together the different parts of stories in order to make connections. ${ }^{27}$ Research suggests that grammatical realizations, i.e. language, can take two possible forms, based upon a participants' perspective: 1) private, first-person speech, in which participants refer to themselves as the experiencers of the task, and 2) public, third-person speech, in which participants do not make explicit references to themselves; instead, they narrate an account of the physical interactions with the object or environment. ${ }^{28}$ Through watching the interviews with participants, it became clear that their responses could be categorized into three perspectives. Based upon this first-level coding, the three pattern codes were established ${ }^{29}$ : body-based perspective, angle-based perspective, and dual perspective, respectively.

While it cannot be known for sure the various perspectives that participants were adopting, coding their language and gestures provide a strong indication as to how they were thinking and developing the angle concept. Multiple studies about embodied cognition have utilized coding in a similar manner in order to learn about participants' thinking. ${ }^{1,7,8,28}$ Participants' perspectives were coded as follows:

- Body-based perspective: Participants' verbal responses referenced their bodies, particularly the positions or movements of their arms. A common explanation that would be categorized as such might begin with, "My arms make the screen..." or, "I move my body to make the screen...".

- Angle-based perspective: Participants' verbal responses referenced the angle on the screen and the ways in which the positions and/or movements of the angle were being altered in order to change the screen's color. A common explanation that would be categorized as such might begin with, "The angle makes the screen..." or "The arrows change the color of the screen when...".

- Dual perspective: Participants' verbal responses referenced their body and the angle on the screen. A common explanation that would be categorized as such might begin with, "My arms move in this way which causes the angle to change the screen...". Furthermore, if a participant referenced the angle on the screen but was glancing at his/her body, a dual perspective was coded, since participants were focusing upon the angle while maintaining awareness of their bodily movements.

It has been demonstrated how gestures provide further means for analysis of perspective-taking during a body-based task. ${ }^{7,} 30 \mathrm{~A}$ first-person perspective incorporates the speaker's body into the gesture and sees the event as if s/he is the person performing the act, whereas a third-person perspective excludes the speaker's body from the gesture, so the hands act as the character as a whole. ${ }^{30}$ In this study, whenever participants looked at their body while speaking, it was implied that they had some awareness of their bodies, so their perspective was categorized as either body-based or dual. Additionally, participants' utterances were coded as 
being the dual perspective if they were describing an angle on the screen but were gesturing in a body-based fashion, such as pointing with their fingers to represent the arrows, moving their arms in a circular motion like the protractor, and/or continually glancing down at their bodies.

After each of participants' utterances were categorized as a body-focused, angle-focused, or body-and-angle-focused, all of the conditions that contributed to the first-level $\operatorname{codes}^{29}$ were analyzed using video recordings and transcripts of the interviews. The interviewer prompts were categorized as being (a) Scripted, deriving from the protocol, (b) Clarification, asking a student to clarify an idea, (c) Elaboration, asking a student to expand upon an idea, (d) Re-voice, repeating what the student said, or (e) Unprompted, the student offering a response without being prompted by the interviewer. Furthermore, the physical environment of the task was categorized according to the conditions of each stage, in which there was nothing on the screen during Stage 1, arrows in Stage 2, a protractor in Stage 3, and long arrows (with the protractor) in Stage 4.

\section{RESULTS}

\section{Case Studies}

Student 1: Jace

Jace, a nine-year-old boy in third grade, had never played with a Wii before, although he had used a Kinect. He did not have a Kinect at his house, however, so he did not use it frequently. For sports, Jace had participated in both dance and cheerleading and indicated that on a scale of 1 to $5(1=$ low, $5=$ high $)$, his preference towards mathematics was a 5 .

On the pre-test, Jace earned a score of 9. Despite his relatively high initial score, he still managed to improve by earning a score of 10 on the post-test. Because of Jace's high scores on both assessments, his interview was analyzed in order to gauge how students' thinking during the body-based task might enable them to achieve high scores.

During Stage 1 of the interview, Jace demonstrated a body-based perspective by consistently referencing the positions and movements of his body; there were not any angles on the screen, and the interview protocol directed participants to respond by referencing their bodies. Although Jace was not always initially correct with his contentions about how to make the screen each color, after moderate prompting from the interviewer, he was able to elaborate upon his responses and revise his original contentions. Jace quickly caught on to the sequence of colors and thought of his arms as lower and higher rungs on a ladder that would turn the screen each color. For example, to make the screen pink (an acute angle), Jace's arms needed to be placed low, whereas to make the screen dark blue (a straight angle), his arms needed to be high. Through this ladder metaphor, he was able to relate the sequence of colors to the positions of his arms.

During Stage 2 of the interview, when short arrows were added to the screen, a majority of Jace's responses shifted from being body-focused to angle-focused. This could partially have been attributed to the fact that the interview protocol no longer placed such a strong emphasis on bodily movements, like in the first stage. Jace expressed multiple metaphors as he grappled with how the angle was related to the color of the screen. When he was asked to make the screen pink (an acute angle), he explained how the angle looked like the top of a triangle, and then after the interviewer repeated his statement, he elaborated that the arrows looked like the top two lines of a triangle. When he was asked to make the screen yellow (a right angle), Jace thought that the angle looked like a normal pyramid, and that it looked like a bow when he was trying to make the screen light blue (an obtuse angle). When asked about dark blue (a straight angle), Jace returned to a body-based perspective by explaining how you have to "put your arm straight up" and then further noted how it "is just like a line", thereby returning to an angle-based perspective.

Stage 3 of the task, in which a protractor was added to the screen, supported Jace's development of the angle-based perspective. He quickly noted that in order to make the screen pink (an acute angle), the measure of the angle needed to be from 0 to 90 degrees. And then, without being prompted by the interviewer, Jace quickly and correctly listed the numerical degrees that described the other three types of angles and corresponding screen colors. Although he had temporarily abandoned a body-based perspective, he understood the "rules" for making the screen each color by correctly identifying the relationship between the color of the screen and angle measure.

Finally, Jace's perspectives were the least consistent during Stage 4 of the task, as he fluctuated between body-focused and anglefocused responses, and, several times, indicated body-and-angle-focused responses. In the following dialogue between Jace and the interviewer, he initially responds from an angle-based perspective, then shifts to a body-based perspective, and then briefly includes the dual perspective, by responding linguistically with an angle-focused response while simultaneously gesturing with his arms to represent the movement of the protractor:

Interviewer: And so let's look at yellow. What can you do to make yellow?

Jace: $\quad$ Go to 90 .

Interviewer: $\quad$ Go to 90 ? 


Jace:
Interviewer:
Jace:
Interviewer:
Jace:

Yeah. You should go like this.

And when you say like this, what are you doing with your-

Putting one out.

Putting one out? Okay.

Because the other one just moves the scale [gestures the movement

of the protractor with his arms].

Jace was able to explore all three types of perspectives by responding to merely one color.

For the remaining questions of Stage 4, Jace alternated between an angle-based and body-based perspective by either referencing the degree measure of the angle, or the position of his arms. Even though he never fully embraced the dual perspective, it is clear that he was conscious of both perspectives, due to the ease with which he fluctuated from one to another during the last stage of the task, when the protocol prompts were relatively open-ended.

\section{Student 2: Abdul}

Abdul, a nine-year-old boy in third grade, had never used a Wii or Kinect before and did not own either of them at his house. He indicated that he played sports, and felt ambivalent about mathematics, as he rated it a 3, on a scale from 1 to 5.

On the pre-test, Abdul earned a score of 2 and did not demonstrate substantial learning gains as a result of the body-based task, since his score only increased to a 3 on the post-test. While Abdul might have acquired new knowledge by participating in the body-based task, such knowledge was not reflected in his minimal gain from pre- to post-test. Consequently, his patterns of perspective-taking become important for gaining insight as to why his test scores reflected little improvement.

During Stage 1 of the task, Abdul primarily explored a body-based perspective; his responses were nearly exclusively related to the movements and positions of his arms. As in the case with Jace, this type of response would be expected during the first stage, because the protocol questions prompt participants to consider how their bodies manipulate the screen's color. Additionally, there are not any angles on the screen for participants to reference. In general, during this stage and throughout much of the rest of the interview, Abdul was not particularly articulate and seemed to struggle with verbally expressing his ideas. His responses were often brief and lacked supporting detail and metaphor. Many of his responses during Stage 1 consisted of utterances such as, "Putting it straight", or, "The other arm", as well as others that were clearly body-focused but did not reveal a wealth of information regarding how he was thinking.

When the arrows were added to the screen for Stage 2 of the task, Abdul's perspective appeared to shift to being angle-based, since many of his responses exclusively referenced the angle. While Abdul was experimenting with making the screen light blue (an obtuse angle), the interviewer prompted him to elaborate on a brief response, and he included the metaphor, "They look like a clock." He later clarified that he meant the angle on the screen looked like a clock, although his clock metaphor was not referenced again at all during the remainder of the interview. Otherwise, during this stage, Abdul's responses continued to lack significant content, and while they were angle-focused in nature, they did not greatly reveal how he was thinking about the angles' impact on changing the screen each color.

Stage 3 of the body-based task included the addition of the protractor, and this did not encourage Abdul to make significant gains in correlating degree measures to the color of the screen. When asked how to make the screen pink (an acute angle), his response demonstrated the dual perspective, since he said, "...one hand is pointing in the middle of 60 and 45 and the other is just pointing at zero." By using his body to describe how to numerically change the angle, he was encapsulating both a body-based and anglebased perspective - in other words, the dual perspective. However, following this response, he retreated to replying with brief, nearly incomprehensible responses that either referenced solely his body or solely the angle. His responses that were anglefocused were not entirely correct, since, for example, he believed that making the screen yellow (a right angle) involved producing a 60-degree angle.

During Stage 4, the lengths of the arrows were increased, and Abdul articulated either body-focused or angle-focused responses, but never both. In fact, his responses hardly contained enough detail to be classified as having explored either perspective. For the last question, when he was asked to make the screen dark blue (a straight angle), he simply responded, "I don't know."

Student 3: Tony

Tony, an eight-year-old boy in third grade, had played with both a Wii and Kinect before, although he only had a Wii at his house. He indicated that he was regularly involved in sports, including swimming and biking, and expressed his dislike for mathematics by giving it a preference rating of 1 , on a scale from 1-5. 
On the pre-test, Tony earned a score of 4, and then notably improved to receive a score of 10 on the post-test. Tony's scores suggest that the body-based task was conducive to his learning and allowed him to demonstrate an improved understanding of elementary angle concepts.

In Stage 1 of the body-based task, Tony's responses were rich with a variety of metaphors that he was eager to explain and expand upon. As with Jace and Abdul's, Tony's responses during the first stage were entirely body-focused, as he continually referenced how his arms played a role in causing the screen to change each color. Tony's enthusiastic energy allowed for a rich dialogue between him and the interviewer, and he showcased his understanding through extensive verbalizations. When asked to make the screen pink (an acute angle), he believed that his body looked like a stick, and then immediately decided that a rocket was a more accurate depiction. By the time he was asked to contend how to make the screen yellow (a right angle), he developed the notion that he was making his arms into different forms of the letter T. For example, to make the screen yellow, he argued that he was "a sloppy T", whereas to make the screen light blue (an obtuse angle), he was "a higher T." By the time he was required to make the screen dark blue (a straight angle), he established that he was "just a T", and even corrected the interviewer when she asked if he was a sloppy T. "So, I never said sloppy T. I just said T," Tony remarked. His body-based perspective was extremely evident through this developed metaphor of the letter $\mathrm{T}$ that needed to be in various forms in order to change the screen each color.

The metaphors continued to develop during Stage 2 of the task, except, unlike Jace and Abdul, Tony remained in a body-based perspective, even though he still integrated many angle-focused responses. When asked how to make the screen yellow (a right angle), Tony referenced the hands of a clock and believed that making the arrows into the time of 4:35 enabled the screen to be yellow. For light blue (an obtuse angle), Tony thought that the angle looked like a bird flapping its wings. This is evidence of the vivid language Tony sought to articulate in his responses; rather than saying the angle looked like a bird, he developed the idea further that it was a bird in a specific stage of movement.

During Stage 3 of the task, Tony encapsulated both body-based and angle-based perspectives, thus resulting in the dual perspective. A true dual perspective means that a student is able to understand the connection between body and concept, or, in this case, the relationship between the movement of one's arms and the angle on the screen. The degree measures of the protractor were arguably vital in helping Tony to establish this connection between the two entities. Tony's ability to develop the dual perspective is evidenced by the following dialogue between him and the interviewer:

Interviewer: What can we do to make gold? (Note: The interviewer would always ask the participant what s/he wanted to call each color. In this particular interview, gold was equivalent to yellow, and violet was equivalent to dark blue.)

Tony: $\quad$ Make it so one hand is on zero and the other is on 90.

Interviewer: One's on zero, one's on 90?

[Unrelated conversation]

Interviewer: And what about light blue? What do you think of light blue?

Tony: Halfway between-put one arm at zero and the other arm between

90 degrees and 135 degrees.

Interviewer: $\quad$ Okay one arm to 135 degrees and 90 degrees. And what about dark blue-violet. What do you do to make violet?

Tony: Violet is my friend. I think it's one hand on zero and the other hand's on 180 degrees. Just make a straight line.

Even though Tony's assertions were not always completely accurate (he was not entirely correct about the angle measure for light blue, for example), he still recognized the relationship between his arms and the angle on the screen.

While maintaining the dual perspective, Tony's metaphors continued into the final stage of the body-based task, although they were not nearly as elaborate as Stage 3. During Stage 4, Tony mentioned on several occasions that he was being asked the same questions multiple times, so perhaps he was becoming frustrated with the repetitiveness of the scripted questions, and did not feel the need to elaborate in as great detail. He answered nearly every question by explaining how one hand needed to be at a certain degree measure, and the other hand could either be within a range of degree measures or at a specific degree measure, depending upon the color of the screen that was being asked. It was clear during Tony's interview that he understood the interrelatedness between body and angle, and was able to assume multiple perspectives along the course of improving his learning.

\section{Analysis}

Overall, this body-based task did seem to support students' developing understanding of an angle concept. Out of all students (n $=32)$, the mean score increased from the pre-test $(\mathrm{M}=5.25, \mathrm{SD}=2.92)$ to the post-test $(\mathrm{M}=7.0, \mathrm{SD}=2.92)$. Based upon the descriptions of Jace, Abdul, and Tony's interviews, there is not one definitive pattern all students take as they complete the bodybased task. Their case studies detail different ways that students might be able to interact with the Kinect and try to make sense of 
the relationship between their body and the angle concept. To that end, there are several factors that became prominent throughout the interviews. It appears as if the physical environment, interviewer prompts, and use of metaphors are three factors that can affect students' abilities to assume different perspectives during this body-based task.

Physical environment. Overall, certain design decisions of the body-based task impacted the perspective that participants took. Participants essentially began with a blank slate, and then tools were progressively added to the screen. During the first stage, the screen was completely blank, aside from continually changing between the four colors. As illustrated in the three students' narratives, each of them began the task in a body-based perspective. Their responses consistently referenced the position and movement of their arms, and the notion of an angle was essentially nonexistent during this stage. It would almost seem illogical for participants to experience any perspective besides a body-based one during this part of the task since there were no tools whatsoever for them to reference. At this early point, it might not even have been clear to participants that angles were the overarching theme. Since all abstractness had been depleted and participants were left with a blank screen (in addition to their physical bodies), the environment limited the perspective. For this reason, coinciding with the scripted prompts, participants remained in a body-based perspective during the entire first stage of the task.

As each new stage of the task was reached, an additional tool was added to the screen, with the intention of allowing participants to utilize it in order to enhance understanding. Stage 3 was one of the most interesting parts of the interview in terms of the physical environment because a protractor was revealed. Prior to the interview, many participants admitted they did not know what a protractor was, had never used one before, or only had a vague idea of its capabilities. During Stage 3, after the interviewer explained how the protractor measures angles, participants were given about 30 seconds to freely explore with it, before being asked the standard questions again about how to make the screen each color. The interviewer just asked participants how to make the screen a certain color; there was no reference to the angle or to their bodies in the prompt. It became the responsibility of the student to internalize the purpose of the protractor and decide the manner in which s/he wanted to respond.

For Jace, a student who had already showcased his prior knowledge about angles on the pre-test, the addition of the protractor was useful in affirming his understanding of angles, since every response he offered was angle-focused. For the first time in the interview, he did not need to be prompted about how to make the screen each color, most likely since the protractor helped him to place a numerical value to the conceptual ideas that he had been developing during the previous two stages. The dialogue between Jace and the interviewer during Stage 3 proceeded as follows:
Interviewer:
Okay, so what can you do to make that pinkish-purple?
Jace:
It can be 45, so zero to-it keeps spinning.
Interviewer:
And if you bring your arms up in front of you now, if you bring it
back to the side?
Jace:
It's from 90 to zero.
Interviewer:
From 90 to zero.
Jace:
And then yellow is only 90 .
Interviewer:
Yellow is only 90?
Jace:
Yeah, and then blue is from 90 to 180. And then purple is only 180.

The interviewer did not prompt Jace in any way to respond from this perspective, so it can be deduced that the protractor was primarily responsible for Jace's angle-based perspective during this stage. Clearly, there were times during the interview when the environment was solely responsible for participants' perspective-taking.

Interviewer prompt. The protocol was designed with certain objectives in mind, one of which included scaffolding students to make connections between their bodies and the angle concept. Because of this, the verbal cues during each of the four stages were intentionally meant to prompt students to adopt different perspectives. Based on the aforementioned interviews, these prompts frequently played a role in the students' explorations of a particular perspective.

During Stage 1, there was an absence of tools on the screen and all of the questions were directly focused upon bodily movements, thus we expected participants to remain exclusively in a body-based perspective. During Stage 2, when the arrows were added to the screen, we expected participants to shift to an angle-based perspective, in hope that they would see the connection between body and angle. Therefore, much of the questions during Stage 2 were meant to prompt an angle-based perspective. The structure of the interview prompts seemed to play a role in Tony's development of the angle concept since he initially began Stage 2 by describing how the positions of his arms affected the color of the screen. However, because participants were supposed to shift their perspective during this stage, the interviewer posed questions to Tony that began to divert his attention away from his body and more towards the angle. The following conversation took place:

Interviewer: I'm going to ask you about pink again. What can you do to make it pink?

Tony:

I forget. Oh yeah. Be a-what some people call an airplane. 
Interviewer: What some people call an airplane? If you use the arrows on the screen, what are the-describe what's happening. What are the arrows doing?

Tony: The arrows are looking like-the arrows look like they're at their lowest position without just touching.

Interviewer: Okay. Lowest position without touching.

Without the interviewer prompting Tony to narrow his focus to the arrows on the screen, he may not have referred to them in his verbal responses, and could have potentially remained in a body-based perspective. Since Tony's scores on the pre- and post-tests increased so substantially, it is interesting to consider if his success can be attributed to the interviewer prompt, since it changed the perspective he had been experiencing.

Similarly, the same interview prompt at the identical stage in the task also seemed to help shift Jace's perspective. When he was first asked how to make the screen pink (after the arrows were added to the screen), he started to recall prior knowledge and thought that it was an acute angle. Although he was correct, classifying angles was not a desired learning outcome of the task, so in order to readjust his perspective to the angle itself rather than being an observer classifying an angle, a direct question about the angle on the screen was necessary. His focus was shifted and he assumed an angle-based perspective. Although there were surely other moments in the interviews when the scripted prompts played a role in maneuvering students' perspective-taking, the beginning of Stage 2 seemed to be the most pivotal for targeting students' thinking on a different perspective than what they had been grappling with just moments before.

Use of metaphor. The use of metaphors appears to support the development of the dual perspective. This was particularly evident in Tony's case. Compared to Jace and Abdul's, Tony's metaphors were much more frequent and tended to be articulated in far greater detail.

Stage 1 of Tony's interview included body-based metaphors that ranged from projectiles in motion to unique ways of writing letters. Tony's initial interaction with the Kinect was verbalized as such:

Interviewer: What do you do with your body to make that pink?

Tony: I'm just being a stick.

Interviewer: You're just being a stick? Is there another way you can make pink?

Tony: Being a stick. Being a rocket, I guess.

Interviewer: $\quad$ A rocket? Can you say more about that?

[Unrelated conversation]

Tony: I don't know if you've ever seen the NASA ships, but normal一not

ones with any- like flybys or satellite launchers. They are straight

with little tips at the top. That's what I mean.

Upon first sharing a metaphor for the position of his body, when Tony was asked how else to make pink, he possibly internalized that question as meaning another metaphor to represent the position of his arms in relation to his body. Then, his detailed explanation of what he meant by being a rocket demonstrates that he was perfectly aware of his body (thus taking on the bodybased perspective) and was able to make a connection to something that he had seen before in life: a rocket.

Research has suggested that "knowing mathematics, really knowing it, means understanding it". 31 "Understanding" is defined as the ability to see how a concept is related or connected to other things we know. ${ }^{31}$ Tony's astute ability to make connections between his body and objects he had been exposed to created an optimal condition for encompassing the dual perspective in the later stages of the task. Once the arrows were introduced to the screen, Tony had the opportunity to make further connections; except, this time, they were connections between his body and the angle, resulting in the dual perspective. Tony was the only one of the three students who truly encapsulated the dual perspective. Despite not being completely correct with every assertion during the third stage, Tony consistently verbalized the position of his arms in relation to the numerical value of the degrees that changed the screen each color. This proved to be a more challenging connection for other students to make.

\section{DISCUSSION}

The body-based task in this study was designed so that participants could adopt the perspectives in a specific manner that we hypothesized would be conducive to learning. Based upon observation and analysis of the three selected students, it suggests that the body-based task was properly designed to support these participants' transitions through various perspectives. Each of the three students endured Stage 1 with a body-based perspective and then focused on an angle-based perspective during Stage 2 . Once the protractor was introduced, there were some differences in the perspectives each of the three students occupied, yet it was clear that they centered on the perspective that was most salient in their minds. For Tony, the protractor greatly influenced his perspective, as it provoked him to relate the position of his arms to the degree measure of the angle. For Abdul, a student who 
struggled throughout the duration of the task, once the questions were less directed at a certain perspective, he retreated to a body-based perspective. It was clear that he was not grasping the angle concept, and so he felt more at ease with his body.

It seems plausible to expect students to be more comfortable with a body-based perspective; they are already familiar with their bodies, and so they are able to use their bodies to make sense of the world and form complex ideas. ${ }^{9}, 11$ By first prompting participants to consider how their bodies were affecting the angle on the screen, we were narrowing their attention and allowing them to explore an entity that they should have felt quite comfortable with. Perhaps, as evidenced by Abdul's interview, if a student was not able to comprehend the angle concept, then he or she would resort to using a body-based perspective.

Mastering the body is helpful in order for development and learning to occur. ${ }^{32}$ More specifically, whenever students are in the midst of learning mathematics, they are forced to constantly question the physical environment, which facilitates changes in their bodily movements. ${ }^{33}$ The ease with which students make those changes could be related to how deeply they master the new and unknown concept.

Along with establishing a strong body-based perspective early on in the task, the use of metaphors seemed to aid the three students in better understanding the angle concept. Metaphors are a simple way to linguistically describe our bodily interactions with the world. ${ }^{10,34}$ If students genuinely understand a concept, then they are capable of expressing connections between the new idea and previous knowledge, ${ }^{31}$ which can take place in the form of metaphors that relate the body to a concept. In the bodybased task, Tony's metaphors were vivid and frequent, and perhaps, his ability to make connections in the first stage was related to his strength in eventually connecting the movement of his body with the angle measure on the screen. On the contrary, Abdul lacked metaphor in his verbal responses, particularly during the earlier stages, so this potentially became a hindrance on his ability to connect different entities later in the interview.

The results of this study were somewhat limited due to the constraint of only analyzing three students. It would be interesting to consider whether other students whose patterns of perspective-taking closely align with Tony's also demonstrate significant learning gains or students whose patterns model Jace's continue to affirm their previously strong angle concept. Similarly, students whose patterns resemble Abdul's during the body-based task might not achieve learning gains. Furthermore, the case studies featured three male students, so a lack of female representation might affect the patterns of perspective-taking. The three students were initially selected blindly, as only their scores on the assessments were considered for inclusion. Therefore, if a larger size of students were to be selected, including both males and females, the results of this specific analysis might be impacted.

Additionally, increasing the sample size would allow further generalizations to be made about how students interact with and learn from a body-based task.

Since so much of mathematics curriculum and instruction hinges on understanding abstract concepts, it is crucial to be mindful of how students can better grasp complex ideas, like angles. This study has suggested that prompting students to explore abstract concepts in a body-based fashion can promote their development of abstract ideas that become pivotal to truly learning mathematics, beyond procedural computations. It provides grounds for mathematics educators to consider how they are approaching instruction in their classroom, and if they are supporting students' intellectual growth of concepts. Finally, if we accept that a body-based perspective is necessary as a first step towards being able to make connections between physical actions and mental representations, then we can further consider specific patterns of perspective-taking that help students to make meaning of abstract concepts.

\section{ACKNOWLEDGEMENTS}

The author thanks Carmen Smith, an exceptional advisor who has helped in countless ways throughout the entire process of producing this work. Thank you, also, to Barbara King and Diana Gonzales for their contributions and support. 


\section{REFERENCES}

1. Nemirovsky, R., and Ferrara, F. (2009) Mathematical imagination and embodied cognition, Educ Stud Math 70, $159-174$.

2. Johnson-Glenberg, M. C., Birchfield, D. A., Tolentino, L., and Koziupa, T. (2013) Collaborative embodied learning in mixed reality motion-capture environments: Two science studies, J Educ Psychol, 19-30.

3. Nathan, M. J., Walkington, C., Boncoddo, R., Pier, E., Williams, C. C., and Alibali, M. W. (2014) Actions speak louder with words: The role of action and pedagogical language for grounding mathematical proof, Learn Ins 33, 182-193.

4. Johnson-Glenberg, M., Birchfield, D., Koziupa, T., Savio-Ramos, C., and Cruse, J. (2012) Seeing it Versus Doing it: Lessons from Mixed Reality STEM Education, in Proceedings of the International Conference of the Learning Sciences: Future of Learning (Freedbody, P., de Jong, T., Kyza, E., and Reimann, P., Eds.), 103-104, University of Sydney/ISLS, Sydney.

5. Ackermann, E. K. (1996) Perspective-Taking and Object Construction: Two Keys to Learning, in Constructionism in Practice: Designing, Thinking, and Learning in a Digital World (Kafai, Y. and Resnick, M., Eds.), 25-37, Lawrence Erlbaum Associates, Mahwah, NJ.

6. Petrick, C. J., and Martin, H. T. (2012) Learning Mathematics: You're it vs. it's it, in Proceedings of the International Conference of the Learning Sciences: Future of Learning (Freedbody, P., de Jong, T., Kyza, E., and Reimann, P., Eds.), 101-102, University of Sydney/ISLS, Sydney.

7. Gerofsky, S. (2011) Seeing the Graph vs. Being the Graph: Gesture, Engagement and Awareness in School Mathematics, in Integrating Gestures (Stam, G., and Ishino, M., Eds.), 245-256, John Benjamins, Amsterdam.

8. Smith, C., Berland, M., and Martin, T. (2014) Playing Robot: Exploring how Students Alternate Perspectives in IPRO, in Learning Technologies and the Body: Integration and Implementation in Formal and Informal Learning Environments (Lee, V., Ed.), 132-148, Routledge, New York.

9. Anderson, M. L. (2003) Embodied cognition: A field guide, Artif Intell 149, 91-130.

10. Lakoff, G., and Johnson, M. (1999) Philosophy in the Flesh: The Embodied Mind and its Challenge to Western Thought, Basic Books, New York.

11. Barsalou, L. W. (2008) Grounded cognition, Annu Rev Psychol 59, 617-645.

12. Kirsh, D., Complementary Strategies: Why we use our Hands when we Think, http:/ / adrenaline.ucsd.edu/kirsh/articles/cogsci95/cogsci95.html (accessed Dec 2015).

13. Abrahamson, D., Trninic, D., and Gutierrez, J. F. (2012) You Made it! From Action to Object in Guided Embodied Interaction Design, in Proceedings of the International Conference of the Learning Sciences: Future of Learning (Freedbody, P., de Jong, T., Kyza, E., and Reimann, P., Eds.), 101-102, University of Sydney/ISLS, Sydney.

14. Walkington, C., Srisurichan, R., Nathan, M., Williams, C., Alibali, M., Boncoddo, R., and Pier, L. (2012) Grounding Mathematical Justifications in Concrete Embodied Experience: The Link Between Action and Cognition, in Paper presented at the Annual Meeting of the American Educational Research Association, Vancouver, Canada.

15. Piaget, J. (1952) The Origins of Intelligence in Children, International Universities Press, New York.

16. Lindgren, R., Aakre, A., and Moshell, M. (2012) You're the Asteroid! Body-Based Metaphors in a Mixed Reality Simulation of Planetary Astronomy, in Proceedings of the International Conference of the Learning Sciences: Future of Learning (Freedbody, P., de Jong, T., Kyza, E., and Reimann, P., Eds.), 101-102, University of Sydney/ISLS, Sydney.

17. Byrne, P., and Becker, S. (2008) A principle for learning egocentric-allocentric Transformation, Neural Comp 20, $709-737$.

18. Mitchelmore, M. C., and White, P. (2000) Development of angle concepts by progressive abstraction and generalization, Educ Stud Math 41, 209-238.

19. Thompson, P.W. (2008) Conceptual Analysis of Mathematical Ideas: Some Spadework at the Foundation of Mathematics Education, in Proceedings of the 32nd Annual Conference of the International Group for the Psychology of Mathematics Education (Figueras, O., Cortina, J. L., Alatorre, S., Rojano, T., and Sepulveda, A., Eds.), 45-64, Morelia, Mexico.

20. Douek, N. (1998) Analysis of a Long Term Construction of the Angle Concept in the Field of Experience of Sunshadows, in Proceedings of the 20th Annual Conference of the International Group for the Psychology of Mathematics Education (Olivier, A., and Newstead, K., Eds.), 264-271. Stellenbosh.

21. Mitchelmore, M. C., and White, P. (1998) Development of angle concepts: A framework for research, Math Educ Res J 10, 427.

22. Clements, D. (2003) Teaching and Learning in Geometry, in A Research Companion to Principles and Standards for School Mathematics (Kilpatrick, J., Martin, W. G., and Schifter, D., Eds.), 151-178, National Council of Teachers of Mathematics, Reston, VA.

23. Goldin, G. (2000). A Scientific Perspective on Structured, Task-Based interviews in Mathematics Education Research, in Handbook of Research Design in Mathematics and Science Education (Kelly, A., and Lesh, R., Eds.), 517-545, Lawrence Erlbaum Associates, Mahwah, NJ.

24. Patton, M. (2002) Qualitative Research and Evaluative Methods, Sage Publications, Thousand Oaks, CA.

25. Yin, R. (2013) Case Study Research: Design and Methods, Sage Publications, Thousand Oaks, CA.

26. Smith, C., King, B., Gonzalez, D., and Paul, N. (2015) The Role of Action in the Development of Angle Concepts, paper presented at the annual meeting of the American Educational Research Association, Chicago. 
27. Glesne, C. (2010) Becoming Qualitative Researchers: An Introduction, Pearson, Boston.

28. Ochs, E., Gonzalez, P., and Jacoby, S. (1996) "When I Come Down I'm in the Domain State": Grammar and Graphic Representation in the Interpretive Activity of Physics, in Interaction and Grammar (Ochs, E., Schegloff, E., and Thompson, S., Eds.), 328-369, Cambridge University Press, Cambridge.

29. Miles, M. B., and Huberman, A. M. (1994) Qualitative Data Analysis, Sage Publications, Thousand Oaks, CA.

30. McNeill, D. (1996) Hand and Mind: What Gestures Reveal About Thought, The University of Chicago Press, Chicago.

31. Hiebert, J., Carpenter, T. P., Fennema, E., Fuson, K. C., Wearne, D., Murray, H., Olivier, A., and Human, P. (2000) Making Sense: Teaching and Learning Mathematics with Understanding, Heinemann, Portsmouth, NH.

32. Dewey, J. (1998) How We Think: A Restatement of the Relation of Reflective Thinking to the Educative Process, Houghton Mifflin Company, Boston.

33. Nemirovsky, R., Borba, M., and Dimattia, C. (2004) PME Special Issue: Bodily activity and imagination in mathematics learning, Educ Stud Math 57, 303-321.

34. Gibbs Jr., R. W., Lima, P. L. C., and Francozo, E. (2004) Metaphor is grounded in embodied experience, J Pragmat 36, 1189 1210.

\section{ABOUT THE STUDENT AUTHOR}

Tegan Garon recently graduated from the University of Vermont with a B.S. in Education, in addition to earning his Vermont teaching license for mathematics, grades 7-12. He graduated from the Honors College, in which his senior thesis evolved into this article. He will be teaching mathematics at a Vermont high school in the upcoming school year.

\section{PRESS SUMMARY}

While students are learning mathematical concepts, such as angles, the movements of their bodies support their learning. As students undergo this learning process, they may assume various perspectives, namely a first-person perspective, where they take on the role of the mathematical object, and a third-person perspective, where they perceive themselves as an observer of the task. Students' abilities to fluctuate between these perspectives can have significant effects on their learning gains. 


\section{APPENDIX}

These questions were used for the administration of an identical pre-test and post-test.

1. Draw an angle. Now draw an angle that is different from the first one you drew.

2. Draw an angle that is bigger than this angle.

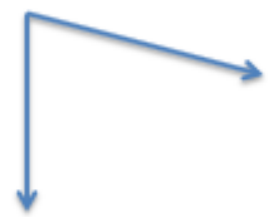

3. Draw an angle that is smaller than this angle.

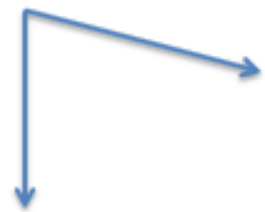

4. Draw an angle that is 90 degrees.

5. Draw an angle that is 30 degrees.

6. Draw an angle that is 150 degrees.

7. Draw an angle that is 180 degrees.

8. Estimate the size of this angle.

$\leftarrow$

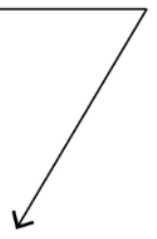

9. Estimate the size of this angle.

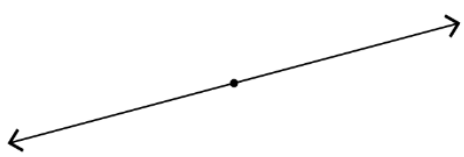

10. Estimate the size of this angle.

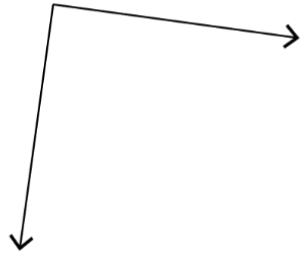

11. Estimate the size of this angle.

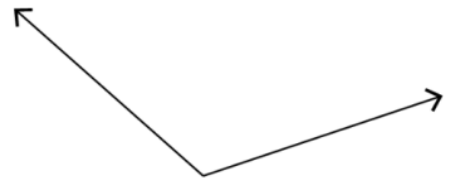




\title{
Affine Symmetry Tensors in Minkowski Space
}

\author{
Isaac Ahern*, Sam Cook \\ Department of Mathematics, University of Alaska, Anchorage, AK
}

Student: iahern@alaska.edu*

Mentor:scook@alaska.edu

\begin{abstract}
Killing vectors are generators of symmetries in a spacetime. This article defines certain generalizations of Killing vectors, called affine symmetry tensors, or simply affine tensors. While the affine vectors of the Minkowski spacetime are well known, and partial results for valence $n=2$ have been discussed, affine tensors of valence $n>2$ have never been exhibited. In this article, we discuss a computational algorithm to compute affine tensors in Minkowski spacetime, and discuss the results for affine tensors of valence $2 \leq n \leq 7$. After comparison with analogous results concerning Killing tensors, we make several conjectures about the spaces of affine tensors in Minkowski spacetime.
\end{abstract}

\section{KEYWORDS}

Affine Symmetry Tensors; Affine Vectors; Killing Tensors; Killing Vectors; Minkowski Spacetime; Dimension; Maple CAS; Lie Derivative; Generalized Killing Tensor

\section{INTRODUCTION}

The study of Killing tensors is the study of generalizations of certain vector fields, called Killing vectors, along algebraic lines. This paper examines tensors occurring on Minkowski space, the space of special relativity. There are 10 fundamentally different geometrical transformations on Minkowski space, called isometries, each with an associated vector field. These are known as Killing vectors of the space, which can be expressed as solutions of a certain equation, Killing's equation. The motivation for the study of affine symmetry tensors comes from examining the solutions to a more general equation than the Killing tensor equation; these generalizations will be examined in more detail in the next section. Essentially, the Killing tensors and the affine vectors of Minkowski space can be characterized as solutions of certain equations similar to the Killing equation, and by generalizing the Killing equation in an appropriate way, we may permit entirely new forms of solutions.

One way to generalize is to seek solutions to the following equation,

$$
\partial_{\left(\alpha_{1}\right.} \partial_{\alpha_{2}} \cdots \partial_{\alpha_{s}} \mathbf{X}_{\left.\alpha_{s+1} \cdots \alpha_{s+n}\right)}=0
$$

whose solutions are called Killing tensors of valence $n$ and order $s,{ }^{1}$ or sometimes generalized Killing tensors. ${ }^{2}$ All of the tensors mentioned above are generalized Killing tensors. The affine symmetry tensors studied in this paper are also a subclass of generalized Killing tensors. In a paper the authors became aware of after this paper was originally submitted, these were called homogeneous generalized Killing tensors in the work of Caviglia, et. al., ${ }^{2}$ where their relationship to the equation of geodesic deviation was studied. More recently, these tensors were studied as a direct generalization of affine vectors. ${ }^{3}$

The goal of the present work is to characterize the number of affine symmetry tensors of a given valence in the Minkowski spacetime. We accomplish this by computation using the software package Maple, for tensors of valence $n, 2 \leq n \leq 7$, which extends what is currently known for these tensors. The code for the valence 4 computation is attached to this article, and illustrative snippets are given in the text below. The code itself is also discussed in a later section, as are ways the code could be modified for similar computations in different spacetimes.

\section{PRELIMINARIES}

Tensors in $\mathbb{M}^{4}$. The Minkowski spacetime $\mathbb{M}^{4}=\left(\mathbb{R}^{4}, \eta\right)$, is the four dimensional flat spacetime of special relativity. The metric tensor $\eta$ on $\mathbb{M}^{4}$ is a valence 2 covariant tensor with components defined by the line element

$$
\mathrm{d} s^{2}=-\left(\mathrm{d} x^{0}\right)^{2}+\left(\mathrm{d} x^{1}\right)^{2}+\left(\mathrm{d} x^{2}\right)^{2}+\left(\mathrm{d} x^{3}\right)^{2} .
$$

We have $\eta=\eta_{a b} \mathrm{~d} x^{a} \mathrm{~d} x^{b}$, where we have used the Einstein summation convention indicating summation over repeated indices. Comparing with the line element given above, we see the components of the metric $\eta$ are $-\eta_{00}=1=\eta_{11}=\eta_{22}=\eta_{33}$, with all others zero. A 4 -vector is a valence 1 contravariant tensor, $\mathbf{X}$, which has components $\mathbf{X}^{\alpha}$, with the index $\alpha$ taking on the values $0,1,2,3$. We can relate a 4 -vector $\mathbf{X}^{\alpha}$ to its dual, the covector, or valence 1 covariant tensor, $\mathbf{X}_{\alpha}$ by

$$
\mathbf{X}_{\alpha}=\eta_{\alpha \beta} \mathbf{X}^{\beta} \text {. }
$$


This is another instance of the Einstein summation. That is, $\mathbf{X}_{\alpha}=\eta_{\alpha \beta} \mathbf{X}^{\beta}=\sum_{\beta=0}^{3} \eta_{\alpha \beta} \mathbf{X}^{\beta}$

A valence $n$ covariant tensor is a multilinear mapping taking $n$ tangent vectors as arguments. A symmetric valence $n$ tensor is one that is invariant under any permutation of its indices; we signify this by $\mathbf{X}_{\alpha_{1} \cdots \alpha_{n}}=\mathbf{X}_{\left(\alpha_{1} \cdots \alpha_{n}\right)}$. Then for any valence $n$ tensor $\mathbf{X}_{\alpha_{1} \cdots \alpha_{n}}$, we can define a corresponding symmetric tensor $\mathbf{X}_{\left(\alpha_{1} \cdots \alpha_{n}\right)}$, the symmetrization of $\mathbf{X}_{\alpha_{1} \cdots \alpha_{n}}$, in the following manner:

$$
\mathbf{X}_{\left(\alpha_{1} \cdots \alpha_{n}\right)}=\frac{1}{n !} \sum_{\sigma \in S_{n}} \mathbf{X}_{\sigma\left(\alpha_{1} \cdots \alpha_{n}\right)}
$$

that is, where the sum is taken over permutations $\sigma$ from the symmetric group $S_{n}$. The Lie derivative of a vector $\mathbf{X}$ in a direction $\mathbf{Y}, \mathcal{L}_{\mathbf{Y}} \mathbf{X}$, can be computed as the Lie bracket, or commutator $[\mathbf{X}, \mathbf{Y}]$ of $\mathbf{X}$ and $\mathbf{Y}$, given by

$$
\mathcal{L}_{\mathbf{Y}} \mathbf{X}:=[\mathbf{Y}, \mathbf{X}]=\mathbf{Y X}-\mathbf{X Y} \text {. }
$$

More precisely, for any smooth function $f$ on $\mathbb{M}^{4}$,

$$
[\mathbf{Y}, \mathbf{X}](f)=[\mathbf{Y}[\mathbf{X}]-\mathbf{X}[\mathbf{Y}]](f)=\mathbf{Y}(\mathbf{X}(f))-\mathbf{X}(\mathbf{Y}(f)) .
$$

Geometrically, the Lie derivative $\mathcal{L}$ is one measure of how vectors, or more generally tensors, on a manifold change.

The isometries $\widetilde{\boldsymbol{x}}$ on $\mathbb{M}^{4}$ are mappings that preserve the metric $\eta$; expressing this via the Lie derivative, we have that $\mathcal{L}_{\mathbf{X}} \eta=0$, where the vector $\mathbf{X}$ is tangent to the isometry $\widetilde{x}$. If we write out the components of this equation, we see it is equivalent to Killing's equation (after Wilhelm Killing) ${ }^{4}$ in $\mathbb{M}^{4}$

$$
\partial_{(\alpha} \mathbf{X}_{\beta)}=0,
$$

where $\partial_{\alpha}=\frac{\partial}{\partial x^{\alpha}}$ is the derivative with respect to the coordinate $x^{\alpha}$.

Generalizations. The solutions of Killing's equation are called the Killing vectors of the space. By analogy, we can generalize Killing's equation, and define a Killing tensor of valence $n$ to be any valence $n$ symmetric tensor $K_{\alpha_{1} \cdots \alpha_{n}}$ satisfying the equation

$$
\partial_{\left(\alpha_{n+1}\right.} K_{\left.\alpha_{1} \cdots \alpha_{n}\right)}=0 .
$$

It is apparent that the number of equations in the system for the Killing tensor grow quickly; counting, we can see that in the system for a Killing tensor of valence $n$ there are exactly

$$
\left(\begin{array}{c}
4+(n+1)-1 \\
(n+1)
\end{array}\right)=\frac{(n+4) !}{(n+1) ! 3 !}
$$

equations. Another generalization of the notion of Killing vectors is to consider the vectors satisfying

$$
\partial_{\alpha} \partial_{(\beta} \mathbf{X}_{\gamma)}=0
$$

These are called affine vectors. It is easy to see that a Killing vector is also an affine vector, since, if $\mathbf{X}$ is Killing, $\partial_{\alpha} \partial_{(\beta} \mathbf{X}_{\gamma)}=\partial_{\alpha} 0=0$. Hence, the space of affine vectors for a spacetime is at least as large as the space of Killing vectors. Geometrically, affine vectors generate affine motions, which are maps that take geodesic curves to geodesic curves while preserving the affine parameter.

A further generalization extending both of the previous notions is that given by the idea of what will be referred to here as an affine symmetry tensor, or more simply as an affine tensor, which is a symmetric valence $n$ tensor satisfying

$$
\partial_{\alpha_{1}} \partial_{\left(\alpha_{2}\right.} \mathbf{X}_{\left.\alpha_{3} \cdots \alpha_{n+2}\right)}=0
$$

As is the case with the Killing vectors, we see that a Killing tensor is also an affine tensor.

We denote the space of Killing tensors of valence $n$ over $\mathbb{M}^{4}$ by $\mathcal{K}_{n}\left(\mathbb{M}^{4}\right)$, and the space of affine tensors of valence $n$ over $\mathbb{M}^{4}$ by $\mathcal{A}_{n}\left(\mathbb{M}^{4}\right)$; as the context of working with $\mathbb{M}^{4}$ is clear, we shall also make use of the abbreviations $\mathcal{K}_{n}$ and $\mathcal{A}_{n}$. By previous observations then, we have that for any $n>0, \mathcal{K}_{n} \subset \mathcal{A}_{n}$.

We will give a short summary of some known results in the following section. Following this, we discuss the algorithm used to enumerate the affine tensors, and discuss the conclusions and conjectures we drew from the algorithmic output.

\section{SUMMARY OF KNOWN RESULTS}

Size of $\mathcal{K}_{n}$. Vector spaces of Killing tensors in spaces of constant curvature have been studied independently by Tacheuchi, ${ }^{5}$ Delong, ${ }^{6}$ and Thompson. ${ }^{7}$ We will follow the conventions of Thompson; he showed the dimension of the vector space consisting of valence $n$ Killing tensors in $\mathbb{M}^{4}$ is

$$
\left|\mathcal{K}_{n}\right|=\frac{(3+n) !(4+n) !}{3 ! 4 ! n !(n+1) !}=\frac{1}{4}\left(\begin{array}{c}
n+3 \\
n
\end{array}\right) \times\left(\begin{array}{c}
n+4 \\
n+1
\end{array}\right)
$$


This is the maximum number of Killing tensors in any 4-dimensional spacetime. In fact, if $K_{n}^{m}$ is the dimension of the vector space of Killing tensors, where $m$ is the dimension of the ambient space $\left(\mathrm{M}^{4}\right)$ and $n$ is the valence of the tensor, then

$$
K_{n}^{m} \leq \frac{(m+n-1) !(m+n) !}{(m-1) ! m ! n !(n+1) !}=\frac{1}{m}\left(\begin{array}{c}
n+m \\
n+1
\end{array}\right) \times\left(\begin{array}{c}
n+m-1 \\
n
\end{array}\right),
$$

and equality is achieved in spaces of constant curvature.

Many papers explicitly calculating the Killing tensors of a given valence in a particular spacetime have been published..$^{7,8,9,10}$ Moreover, the problems of finding the dimension of the vector space of generalized and generalized conformal Killing tensors have been considered by Nikitin and Prilpko ${ }^{1}$ and Eastwood. ${ }^{11,12}$ In this paper, we present a Maple algorithm to compute the affine tensors (and Killing tensors) in $\mathbb{M}^{4}$ for $n=1, \ldots, 7$; an example of this algorithm that calculates the valence $n=4$ tensors is included in the appendix of this paper. We include in this paper the values of $\left|\mathcal{K}_{n}\right|$ and $\left|\mathcal{A}_{n}\right|$ for each valence.

\section{AFFINE TENSORS IN $\mathbb{M}^{4}$}

The algorithm. A brief explanation of the algorithm is given below, which accompanies the attached sample code for the valence 4 affine symmetry tensors given in the appendix. The code is divided into 5 sections. We note that for space considerations, the output of the commands has been suppressed (by use of a colon instead of a semicolon). We have included small snippets of the output below for purposes of illustration.

In section 1 , we load the necessary packages for the computations, and define the spacetime $\mathbb{M}^{4}$ (by its metric $g=\eta$ ) within which we will work. We define the coordinates of the spacetime, and using these and the metric, compute the Christoffel symbols for computation in section 3. It is worth pointing out that the worksheet attached in the appendix can be used as a template for further calculations, involving tensors of other valences (i.e., the case $n \neq 4$ by very slightly modifying the code described below) or in different spacetimes, by modifying the initial data in this section of the code.

In section 2, we construct a generic valence 4 symmetric tensor, whose components we will be concerned with finding. The attached Maple worksheet includes the construction of a valence 4 covariant tensor; however it is possible to generalize the approach in an appropriate manner to construct a tensor of any valence.

In section 3, we build the system(s) of PDE which would like to solve. The Killing tensor equation is built from the symmetrization of the covariant derivative of the tensor. That is the purpose of the first two lines of this section. The ADK command then covariantly differentiates this, which produces the affine tensor equation. The KTSYS and ADESYS commands then tell Maple to explicitly form the corresponding systems of differential equations from these.

Figure 1. First few components of Affine System PDE.

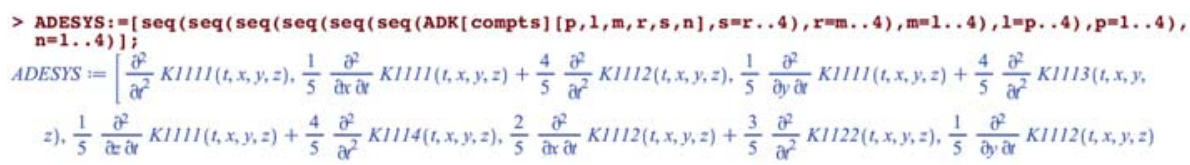

In section 4, we solve the system ADESYS to find the valence 4 (in this case) affine symmetry tensors. This is done using the pdsolve command. The pdetest command is used to verify that the given solutions actually solve the system ADESYS.

Figure 2. 1111 component of general solution, with arbitrary constants shown.

$$
\begin{aligned}
& \text { > sol: =pdsolve (ADESYS); } \\
& \text { sol }:=\left\{K I I I I(t, x, y, z)=\_C l t+\frac{1}{24}-C 2 x^{4}+\frac{1}{24}\left(4 \_C 3 y+4 \_C 4 z+4 \_C 5\right) x^{3}+\frac{1}{24}\left(6 \_C 6 y^{2}+\left(12 \_C 7 z+12 \_C 8\right) y+6 \_C 9 z^{2}\right.\right. \\
& \left.+12 \_C l O z+12 \_C l 1\right) x^{2}+\frac{1}{24}\left(4 \_C l 2 y^{3}+\left(12 \_C l 3 z+12 \_C l 4\right) y^{2}+\left(12 \_C l 5 z^{2}+24 \_C l 6 z+24 \_C l 7\right) y+4 \text { Cl } 78 z^{3}\right. \\
& \left.+12 \_C 19 z^{2}+24 \_C 20 z+24 \_C 2 l\right) x+\frac{1}{24}-C 22 y^{4}+\frac{1}{24}\left(4 \_C 23 z+4 \_C 24\right) y^{3}+\frac{1}{24}\left(6 \_C 25 z^{2}+12 \_C 26 z+12 \_C 27\right) y^{2} \\
& +\frac{1}{24}\left(4-C 28 z^{3}+12 \_C 29 z^{2}+24-C 30 z+24 \_C 31\right) y+\frac{1}{24}-C 32 z^{4}+\frac{1}{6}-C 33 z^{3}+\frac{1}{2}-C 34 z^{2}+\_C 35 z+C C 36, K 1112(t, x, y, z)
\end{aligned}
$$

In section 5, we isolate the components of each solution. Each arbitrary constant in the solution, of the form _C $\mathrm{i}$, corresponds to a different independent solution of the system. So, for each arbitrary constant in the solution, we list here the non-zero components. The commands in this section are sensitive to the number of elements in the lists. Thus, the number of components and the number of solutions must be known for this section. The number of components can be computed. The number of solutions can be found with little effort by using Maple's built in search feature to search the output and determine the maximum index of the coefficients of the solutions, from the output of section 4 . The output can be made visible and searchable by changing the colon to a semicolon in the sol command in section 4. 
Figure 3. Non-zero components of solution corresponding to C2.

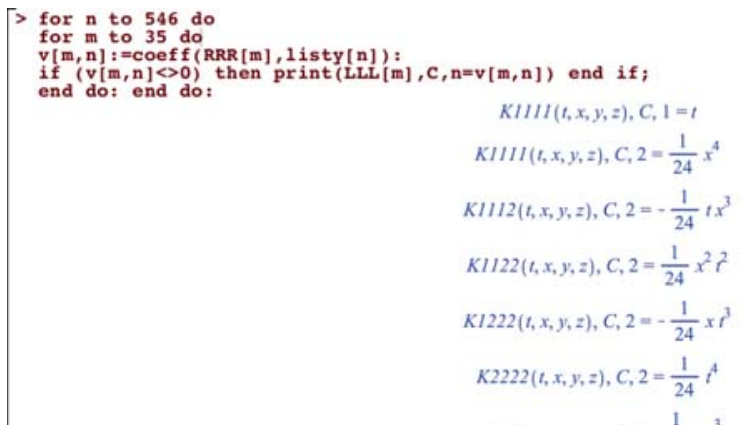

The number of parameters used to describe the solutions, or dimension of the corresponding solution space, was recorded and used to conjecture the dimension, $\left|\mathcal{A}_{n}\right|$, of $\mathcal{A}_{n}$; the data is recorded in the next section (see Table 1).

Results. Information about the number of equations and parameters is collected below:

Table 1. Parameter and Equation Sizes.

\begin{tabular}{|c|c|c|c|}
\hline valence $\mathbf{n}$ & \# Killing Tensor parameters & \# Affine Tensor parameters & \# Affine \& not Killing \\
\hline 1 & 10 & 20 & 10 \\
\hline 2 & 50 & 70 & 20 \\
\hline 3 & 175 & 210 & 35 \\
\hline 4 & 490 & 546 & 56 \\
\hline 5 & 1176 & 1260 & 84 \\
\hline 6 & 2520 & 2640 & 120 \\
\hline 7 & 4950 & 5115 & 165 \\
\hline
\end{tabular}

It appears from the data that the number of Killing tensors of order $n,\left|\mathcal{K}_{n}\right|$, for $n=1, \ldots, 7$ follows the OEIS sequence A006542, ${ }^{13}$ which matches the formula given by Thompson, and that the number of non-Killing affine tensors follows the Tetrahedral number sequence $T_{n}$, which is the OEIS sequence A000292. ${ }^{14}$

Based on the data collected for the affine tensors with valence $n=1, \ldots, 7$, we make the following observation and conjectures: 1. Our data for $\left|\mathcal{K}_{n}\right|$ indicates that

$$
\left|\mathcal{K}_{n}\left(\mathbb{M}^{4}\right)\right|=\frac{1}{4}\left(\begin{array}{l}
n \\
3
\end{array}\right) \times\left(\begin{array}{c}
n-1 \\
3
\end{array}\right)
$$

for $n=4,5,6, \ldots$ It is known that $\left|\mathcal{K}_{n}\right|=\frac{1}{4}\left(\begin{array}{c}n+4 \\ n+1\end{array}\right) \times\left(\begin{array}{c}n+3 \\ n\end{array}\right)$. These two forms are equivalent however, as can be seen by letting. $n \rightarrow n+m=n+4$ where $m$ is the dimension of $\mathbb{M}^{4}$. Then,

$$
\frac{1}{4}\left(\begin{array}{l}
n \\
3
\end{array}\right) \times\left(\begin{array}{c}
n-1 \\
3
\end{array}\right) \rightarrow \frac{1}{4}\left(\begin{array}{c}
n+4 \\
n+1
\end{array}\right) \times\left(\begin{array}{c}
n+3 \\
n
\end{array}\right)
$$

and hence $\left\{\left|\mathcal{K}_{n}\left(\mathbb{M}^{4}\right)\right|\right\}_{n \in \mathbb{N}, n \geq 4}=\left\{\frac{1}{4}\left(\begin{array}{c}n+4 \\ n+1\end{array}\right) \times\left(\begin{array}{c}n+3 \\ n\end{array}\right)\right\}_{n \in \mathbb{N}}$.

2. It appears that

$$
\left|\mathcal{A}_{n} \backslash \mathcal{K}_{n}\right|=\left(\begin{array}{c}
n+4 \\
3
\end{array}\right)=\left(\begin{array}{c}
(n+2)+2 \\
3
\end{array}\right) .
$$

$\left(\begin{array}{c}(n+2)+2 \\ 3\end{array}\right)=T_{n+2}$ also characterizes the Tetrahedral number sequence $T_{n},{ }^{14}$ for $n=1,2, \ldots$ but shifted forward two terms in the sequence. Observations 1. and 2. allow us to make the following conjecture as to the size of $\mathcal{A}_{n}$ :

$$
\left|\mathcal{A}_{n}\right|=\left|\mathcal{A}_{n} \backslash \mathcal{K}_{n}\right|+\left|\mathcal{K}_{n}\right|=\left(\begin{array}{c}
n+4 \\
3
\end{array}\right)\left[1+\frac{1}{4} \times\left(\begin{array}{c}
n+3 \\
3
\end{array}\right)\right] .
$$

In the computations for each $\mathcal{A}_{n}$, we also observe that the components of each tensor for $n=1, \ldots, 7$ are in the form of multivariable polynomials with degree less than or equal to $n$. We conjecture that this is the general form of the tensors in $\mathcal{A}_{n}$, that is, 
the components of each such tensor are multivariable polynomials with degree $\leq n$.

Finally, we conjecture that the number of affine tensors in a space follow a similar inequality to the form that Thompson found that is, that the affine symmetry tensors of valence $n$ for a manifold $\mathcal{M}$ of dimension $m$ are of the order $\mathcal{A}_{n}^{m}$ where

$$
\mathcal{A}_{n}^{m} \leq\left(\begin{array}{c}
n+m \\
m-1
\end{array}\right)\left[1+\frac{1}{m} \times\left(\begin{array}{c}
n+m-1 \\
m-1
\end{array}\right)\right]=\left(\begin{array}{c}
n+m \\
n+1
\end{array}\right)\left[1+\frac{1}{m} \times\left(\begin{array}{c}
n+m-1 \\
n
\end{array}\right)\right],
$$

with the inequality similarly achieving equality in spaces $\mathcal{M}$ of constant curvature, such as $\mathbb{M}^{4}$.

\section{REFERENCES}

1. Nikitin, A. G. and Prilpko, A., (1990) Generalized Killing Tensors and Symmetry of Klein-Gordon-Fock Equations, preprint 90.23, Acad. Sci Ukr SSR, Institute of Mathematics, Kiev, 1 - 59 (Preprint in English available at arxiv.org/pdf/mathph/0506002v1.pdf)

2. Caviglia, G., Salmistraro, F., and Zordan, C., (1982) Geodesic deviation and first integrals of motion, J. Math. Phys., 23, 2346 $-2352$

3. Cook, S. and Dray, T., (2009) Tensor generalizations of affine symmetry vectors, J. Math. Phys., 50, 122506 - 122512.

4. Martin, D., (2002) Manifold Theory: an Introduction for Mathematical Physicists (Horwood), 228 - 238, Simon \& Schuster, Sussex.

5. Tacheuchi, M., (1983) Killing tensor fields on spaces of constant curvature, Tsukuba J. Math., 7, $233-55$

6. Delong, R.P., (1982) Killing tensors and the Hamilton-Jacobi equation, PhD thesis, University of Minnesota

7. Thompson, G., (1986) Killing tensors in spaces of constant curvature, J. Math. Phys., 27, 2693 - 2699.

8. Caviglia, G. and Zordon, C., (1982) Third-Order Killing Tensors in the Schwarzschild spacetime, Gen. Relat. Gravit., 14, 27 30.

9. Ikeda, M. and Kimura, M., (1972) On Quadratic First Integrals in the General-Relativistic Kepler Problem, Tensor N. S., 25, $395-404$.

10. Walker, M. and Penrose, R., (1970) On Quadratic First Integrals of the Geodesic Equations for Type \{22\} Spacetimes, Comm. Math. Phys., 18, $265-274$.

11. Eastwood, M., (2005) Higher symmetry of the Laplacian, Annals of Mathematics, 161, 1645 - 1665

12. Eastwood, M., (2005) Representations via overdetermined systems, Contemp. Math., AMS, 368, 201- 210

13. The On-Line Encyclopedia of Integer Sequences, https://oeis.ong/A006542 (accessed Mar 2016)

14. The On-Line Encyclopedia of Integer Sequences, https://oeis.org/A000292 (accessed Mar 2016)

\section{ABOUT THE STUDENT AUTHOR}

Isaac Ahern is a recent graduate of the University of Alaska, Anchorage, where this research was completed. Isaac has just finished his first year of graduate study at the University of Oregon.

\section{PRESS SUMMARY}

This paper investigates the affine symmetry tensors, an exciting new generalization of Killing vectors, generators of symmetries, in the Minkowski spacetime of special relativity. While partial results are known about the affine symmetry tensors for valence $1 \& 2$, affine tensors of valence $n>2$ have never before been exhibited. In this article, we discuss a computational algorithm to compute affine tensors in Minkowski spacetime, and discuss the results for affine tensors of valence $2 \leq n \leq 7$. These results are used to make several conjectures about the spaces of affine symmetry tensors analogous to statements about the spaces of Killing tensors. 


\section{APPENDIX}

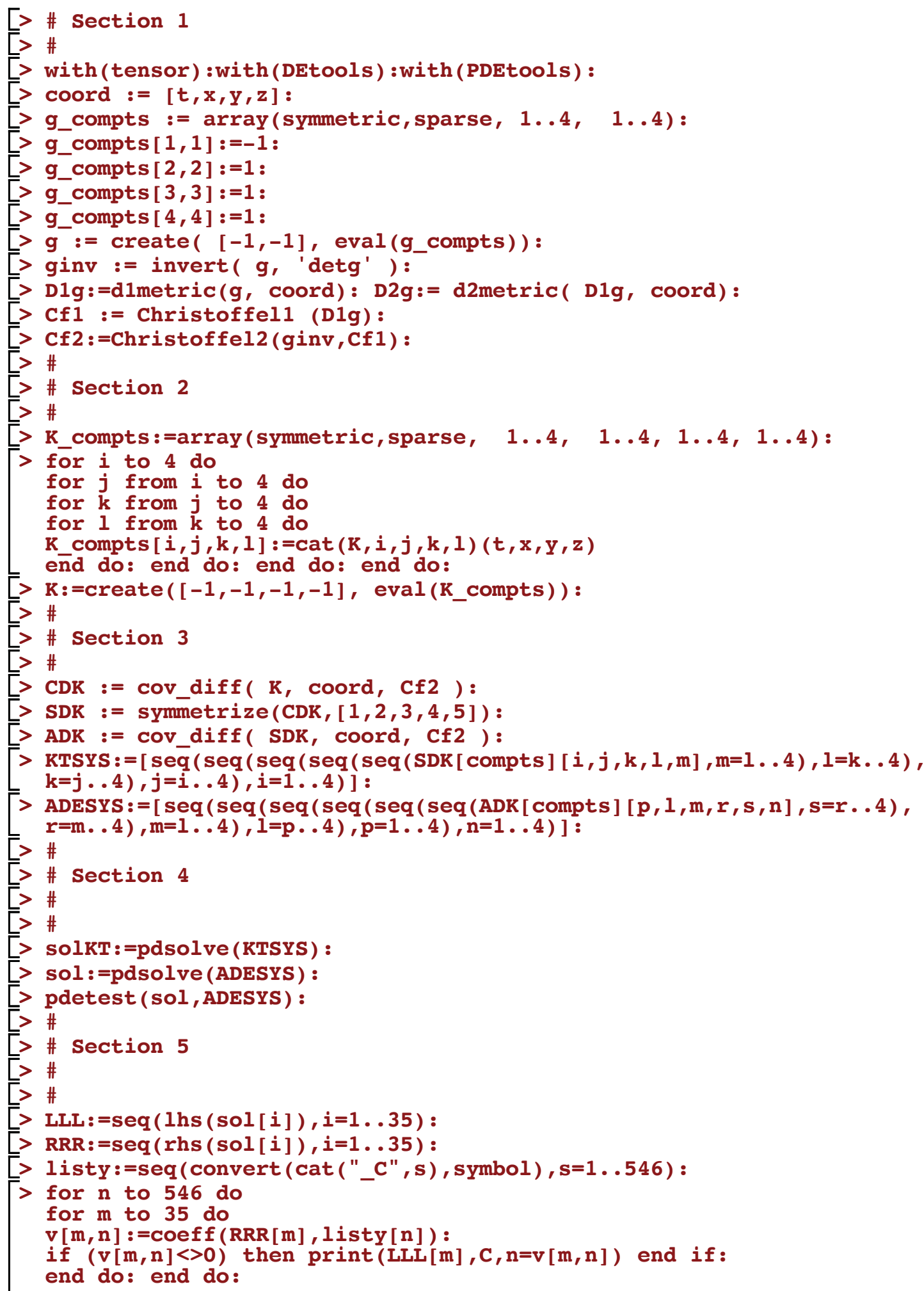




\title{
A Relationship Between Time Perception and State-Anxiety
}

James Brown

Department of Psychology, Coastal Carolina University

jmbrown@g.coastal.edu

\begin{abstract}
Participants in this study were randomly assigned to one of three interval groups of either one, three, or five minutes. All participants were asked to estimate a group-assigned time interval and complete the state portion of the State Trait Anxiety Inventory (STAI). It was hypothesized that higher levels of state anxiety would cause participants to overestimate the passage of time. It was also hypothesized that shorter interval durations would be estimated more accurately than longer interval durations. Results of a $t$ test did not support the first hypothesis. Results of a linear trend analysis did support the second hypothesis $(\mathrm{P}<.05)$. These results indicate that state-anxiety does not cause the passage of time to be overestimated and that interval duration length affects how accurately time is estimated. Implications of this study are important to the understanding of human time-management ability and time estimation errors.
\end{abstract}

\section{KEYWORDS}

Time estimation; Perception; State-anxiety; Attention; Working memory; Emotion

\section{INTRODUCTION}

Our ability to comprehend causal relationships in time is a fundamental component of learning and human behavior. ${ }^{1}$ Humans monitor time by referring to interval durations of seconds, minutes, and hours. Because time is constant, a second will always be a second, an hour an hour, and so forth. However, perception of those interval durations tends to be variable and often inaccurate. ${ }^{2}$ If not for the invention of instruments to measure time, the ability to accurately quantify and monitor how much time has elapsed in the form of some unit of measurement would be quite inconsistent. Unlike most of the neural input the brain receives from the receptors for taste, touch, smell, sight, and hearing, time is not physically sensed, yet the passage of time can be sensed cognitively. ${ }^{3,4}$ According to pacemaker-accumulator models of time perception, time perception is a product of cognitive reinforcement and working memory, and time estimation is made possible by a series of neural signals produced by an internal pacemaker in the brain., ${ }^{3}$ Under pacemaker-accumulator models of time perception, neural signals are collated, counted and compared to stored representations of interval durations to which future durations and time estimations are based (see Figure 1. for depiction of internal time perception model). ${ }^{5}$

\section{MODELS OF TIME PERCEPTION}

Numerous studies continually support cognitive pacemaker-accumulator models of time perception, showing that estimations of durations approximately two seconds or longer rely primarily on working memory and that participants tend to make more time error estimations when they are presented with visual and auditory distractions. ${ }^{6}$ Scalar Expectancy Theory (SET), which is a widely accepted pacemaker-accumulator model of time perception, hypothesizes that intervals of time are perceived when an individual consciously attends to them. ${ }^{3}$ Further, because attention and working memory work conjunctively, any exogenous factors that alter attention, emotional arousal in particular, will also alter the perception of time. ${ }^{7}$ Thus, emotional variables that affect working memory should also affect the perception of time. Decades of electrophysiological studies support this notion, showing that emotional arousal, attention and working memory are neuro-functionally coupled within the anterior cingulate cortex (ACC), a brain structure found to be involved decision-making tasks, impulse control and emotion processing in general. ${ }^{8}$

\section{NEUROPHYSIOLOGICAL CORRELATES}

Providing a proof of concept for a functional link between emotional arousal, attention and working memory is a study that examined the influence of emotional context on attentional resources was indexed using electroencephalogram (EEG). ${ }^{9}$ Eventrelated potentials (ERPs) related to attentional processing and working memory were recorded while participants engaged in a computerized version of the Go/Nogo task modified to include background images either positive, negative or neutral in emotional valence. Statistical analyses of ERPs indicated that the magnitude of ACC signals changed differentially depending on whether participants were exposed to positive or negative emotional stimuli. Further, the presentation of negative emotional stimuli positively correlated with participants having increased reaction time. ${ }^{9}$ 


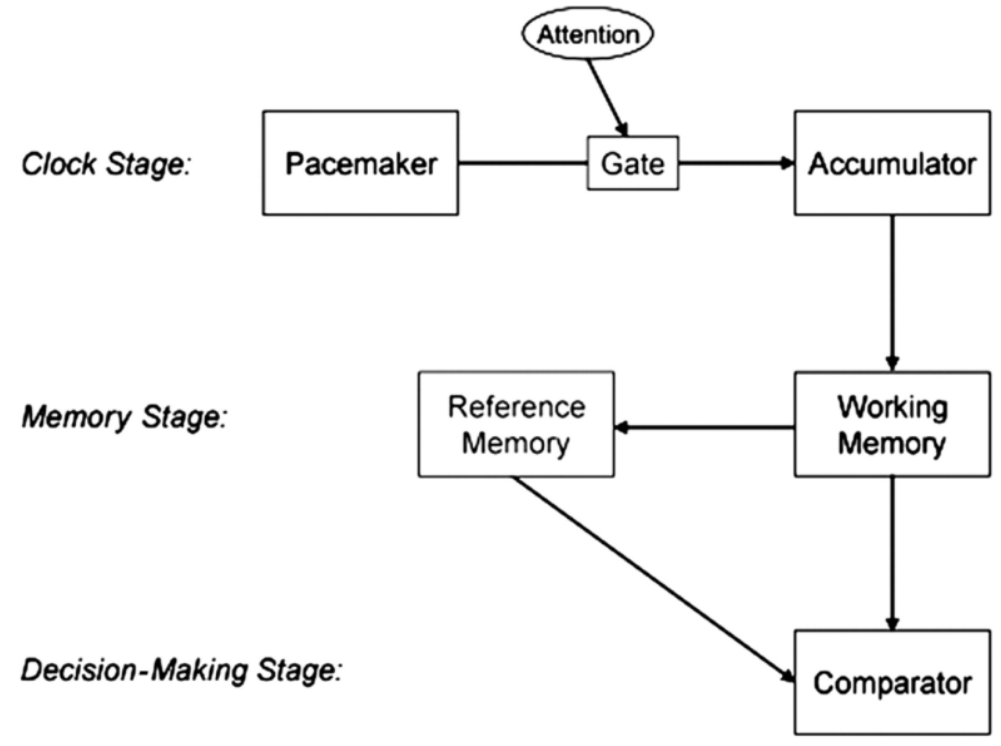

Figure 1. A graphical representation of an internal clock model of time perception. This depiction is from a study by Pencil, Coslett, Aguirre and Chatterjee (2010) and was created to discuss the neural bases for interval timing. ${ }^{5}$

The authors concluded that the neurophysiological effects of high state-anxiety disrupted the attentional resources of the participants because the participants' attentional resources were directed to negative thought processes, thus leading to their performance changes. ${ }^{9}$ Although this was not a time perception study, because time perception is hypothesized to be a process utilizing attentional resources it is reasonable to suggest that if emotional arousal altered attentional processes time perception processes would also have been altered. Previous studies even suggest that meditation, an activity empirically supported to increased awareness and mental clarity, works by engaging the ACC, as well the insula, posterior cingulate cortex (PCC), prefrontal and thalamic cortices, such that attention and emotion are affected. ${ }^{10}$

\section{RELATED WORK}

In a similar vein, studies on meditation have found that calm mental states have the potential to influence time perception. In one study it was found that both inexperienced and experienced meditators exhibited increased post-test performances in a temporal bisection task posttest compared to pretest when they engaged in 10 minutes of meditation prior to engaging in the task. ${ }^{11}$ Additionally, all participants exhibited decreased anxiety, as well as self-reports of slowed perception of time, following meditation. ${ }^{11}$ Demonstrating the effects of increased anxiety was a study that explored the relationship between nicotine withdrawal and duration discrimination ability by having habitual smokers abstain from smoking for 24 hours prior to the study. ${ }^{11}$ Given the wellknown negative psychophysiological effects that accompany nicotine withdrawal (e.g., anxiety, attentional disruption), the authors hypothesized that duration discriminability would be overestimated. ${ }^{12}$ Results of this study indicated that all participants were more likely to overestimate the passage of time when they abstained from smoking and that this effect was stronger in males than females. ${ }^{13}$

\section{TIME PERCEPTION AND EMOTIONAL AROUSAL}

Apart from the indirect evidence, a number of studies directly support the notion that emotional arousal disrupts time perception processes. For example, in one study subjects were administered the State-Trait Anxiety Inventory (STAI) and then asked to engage in a duration prediction task. ${ }^{14}$ Specifically, the participants had to estimate how long it would take to read a five-page manuscript. Results of this study indicated that the highest STAI scores were correlated with greater prediction overestimations. These results were in accord with past research suggesting that anxiety is associated with negative expectation biases, and indicates that individuals with higher levels of anxiety may anticipate greater cognitive processing demands. ${ }^{14}$ Similarly, in another using the same duration prediction task as the previous study, it was found that individuals who are more conscientious are also more likely to overestimate how long it will take them to complete the task. ${ }^{15,16}$ This result suggests a mediating link between anxiety and conscientious; however, the temporal ordering of these variables is not unambiguously dissociable. Given these effects are due to negative emotional biases, these results can be linked back to aforementioned studies in which it was found that negative emo- 
tional stimuli have the potential to attenuate attentional resources. ${ }^{9}$ Taken together, the idea that emotional arousal distorts time perception ability is strongly supported.

\section{DURATION DISCRIMINATION}

Although attentional resources and emotional arousal are interdependent processes mediated by shared brain mechanisms, and those relationships likely impact time perception, different questions remain unanswered: specifically, how accurately are interval durations judged compared to actual clock time durations and how accurately are shorter interval durations compared to longer durations? Providing partial answers to these questions a study examined how accurately interval durations are estimated as a function of interval duration length. ${ }^{17}$ Each participant was asked to estimate each of five time intervals (e.g. 15, 30, 45, 60 and 75 seconds). The results of this study indicated that time estimation was significantly affected by interval duration length and the shorter interval durations were judged more accurately than longer durations. Subsequent studies using similar paradigms indicate that shorter interval durations are judged more accurately than longer durations. ${ }^{18}$ While these studies support the notion that working memory is a key component of time perception, and is possibly the reason for what constrain the ability to estimate time, they do not account for the impact that emotions can have on the ability to estimate time. ${ }^{18}$

\section{PRESENT STUDY}

If attention and emotional arousal are interdependent processes that affect time perception, how those two variables affect one another during the estimation of time over varying interval duration lengths could evince their relationship. The aforementioned studies described that while humans have the ability to track clock time, their perceived time is not necessarily a reflection of actual clock time. The models and theories of time perception discussed primarily focus on the time estimation aspect of time perception. Though humans have the ability to discriminate interval durations accurately, those interval discriminations are susceptible to distortion by an individual's emotional state. In particular, the emotional state of anxiety appears to disrupt an individual's attentional resources. Because emotional states normally persist longer than durations previously studied, studying these effects over prolonged durations will improve further delineate the impact of emotional arousal on the ability to estimate time.

The following study was designed to examine the effect of anxiety on time estimation and to determine if accurate time estimation judgments are affected by interval duration. The interval durations utilized in this study were selected based on their being significantly longer than the durations used in similar studies; the interval durations selected for this study were one, three, and five minutes. The researcher hypothesized that higher levels of state anxiety would affect participants in such a way that they would overestimate the passage of time. In addition, it was also hypothesized that shorter time interval durations would be estimated more accurately than longer durations.

\section{METHOD}

\section{Participants}

Thirty participants (22 female, 8 male) were recruited from psychology classes and the cafeteria at Coastal Carolina University using a non-random convenience sampling technique. Institutional IRB approved this project. Subjects ranged in age from 18 to 27 years $(M=21.3)$. Participants were compensated by their professors with bonus points added to their lowest exam grade.

\section{Materials}

A standard informed consent form was used which was a part of each materials packet. A demographic survey was also used in order to obtain participant's general information such as their sex, age, and academic major. A materials packet also included the state-anxiety portion of the State-Trait Anxiety Inventory (STAI) ${ }^{19}$ The STAI differentiates between two types of anxiety; state anxiety and trait anxiety, where state anxiety and trait anxiety are both defined as emotional conditions that are characterized by apprehension, tension, and fear about a particular situation. ${ }^{19}$ The primary difference between state and trait anxiety is that trait anxiety is a more continuous form of anxiety, while state anxiety is temporary. Furthermore, those with trait anxiety are persistently expectant of situations that might create anxiety, which further exacerbates this condition. The STAI is a self-report assessment survey designed to measure both state anxiety and trait anxiety. There are two sections of the STAI. One section of the STAI is designed to measure state-anxiety and the other section is designed to measure trait-anxiety. Both sections of the STAI contain 20 response items such as "I feel frightened" or "I feel calm". Participants respond to those response items by circling a response from a four-point Likert scale in which one refers to "NOT AT ALL" and four refers to "VERY MUCH SO". Those Likert responses are designed to describe how the participant presently feels. ${ }^{19}$ A Unisex Timex T53151 stopwatch was used to time response intervals.

\section{Procedure}

Data were collected in a lab in the psychology department at Coastal Carolina University. The lab was a small room that was lit with standard fluorescent lighting and it contained one desk and two chairs which were placed at opposite sides of the desk. Upon arrival at the laboratory, each participant was welcomed and asked to be seated. Once seated, the participant was handed a materi- 
als packet that contained an informed consent form, demographic survey, and a copy of the state-anxiety portion of the STAI. Upon completion of their materials, those were collected. Each participant was randomly assigned to one of three interval groups of either one, three, or five minutes. The participant was instructed that he/she would estimate an interval of time and that the use of cellphones, watches, or any other electronic device was not permitted. In addition, the participant was also told he/she could not count aloud or use rhythmic tapping to estimate the time interval.

The participant was instructed that the activity would begin when the researcher said aloud "NOW" and that when the participant estimated the time had elapsed the participant was to say "OK" or "STOP" aloud. The researcher sat across the table from the participant and utilized a stopwatch to record the participant's estimate of the time interval. The stopwatch was activated when the participant said "NOW" and recorded time in seconds until the participant said "STOP". Upon completion of the activity the participant was thanked for his/her participation and escorted from the lab. These procedures were repeated for all 30 participants in this study.

\section{RESULTS}

This study investigated the dependent variables of participant-estimated time intervals and state-anxiety scores. The highest score on the state-anxiety portion of the STAI is 80 and the lowest score is 20. In this study, state-anxiety scores ranged between 20 and 60. The average state-anxiety scores for males was 35.00 and the standard deviation was 12.78 and average state-anxiety score for females was 38.09 and the standard deviation was 10.38. A second dependent variable in this study was an absolute error (AE), and these values represented the difference between participant-estimated time intervals and experimenter-assigned time intervals. The independent measure in this study was the experimenter-assigned time interval. For participants in the one-minute experimenter-assigned time interval group, the average time estimate was 64.00 seconds and the standard deviation was 24.23 seconds. Participants in the three-minute experiment assigned interval group the average time estimate was 170.60 seconds and the standard deviation was 46.09 seconds. For participants in the five-minute experimenter-assigned interval group, these values were 296.00 seconds and 76.50 seconds respectively. (See Table 1 for experimenter-assigned time intervals compared to the average estimate of assigned time intervals.)

\begin{tabular}{|c|c|}
\hline Experimenter-Assigned Time Intervals & $\begin{array}{c}\text { Average Estimate of Assigned Time Inter- } \\
\text { vals }\end{array}$ \\
\hline one minute (60 seconds) & 64.00 seconds \\
\hline three minutes (180 seconds) & 170.60 seconds \\
\hline five minutes (300 seconds) & 296.00 seconds \\
\hline
\end{tabular}

Table 1. Experimenter assigned time intervals compared to the average estimates of assigned time intervals.

It was hypothesized that higher state-anxiety scores would correspond with participant overestimations of time interval durations. The average state-anxiety score for those who overestimated the time intervals was 37.77 and the standard deviation was 8.85 and for those who underestimated the time intervals the average state-anxiety score was 36.12 and the standard deviation was 12.34 . In order to determine if state-anxiety scores differed as a function of over or underestimations of time, an independent-samples one-tailed t-test was calculated to compare scores from 13 participants who overestimated and 17 participants who underestimated their assigned time intervals (refer to Table 2 for group sizes, mean state-anxiety scores, and standard deviations as a function of over or underestimation groups). The results of this test indicated no statistical difference in state-anxiety scores between participants who overestimated and those who underestimated assigned time intervals, $t(28)=-.41, \mathrm{P}>.05$.

\begin{tabular}{|l|l|l|l|}
\hline STP & n & MSA & sd \\
\hline Overestimated & 13 & 37.77 & 8.85 \\
\hline Underestimated & 17 & 36.12 & 12.34 \\
\hline
\end{tabular}

Table 2. Group sizes, mean State-Anxiety Scores, and standard deviations as a function of over or underestimation groups. 
Following the elimination of three extreme outliers, state-anxiety and general time-estimation error were tested by conducting a correlation analysis. The results of that correlation test revealed a significant negative correlation between state-anxiety score and general time-estimation error, $r=-.33, \mathrm{P}<.05$, one-tailed. An even further examination of the data via a curvilinear regression analysis revealed a substantial curvilinear trend with a significant quadratic term, $t(24)=2.26, \mathrm{P}<.05$ (refer to Figure 1 for graph delineating the curvilinear regression analysis).

To test the hypothesis that shorter interval durations would be estimated more accurately than longer durations a linear trend analysis was calculated between time interval duration and the absolute value of the error of time estimations. Results of this test indicated statistical significance for the effect of interval duration length and accuracy of participant-estimated time, $F(1,27)=$ 6.12, $\mathrm{P}<.05$ (refer to Figure 2 for graph delineating the linear trend analysis).

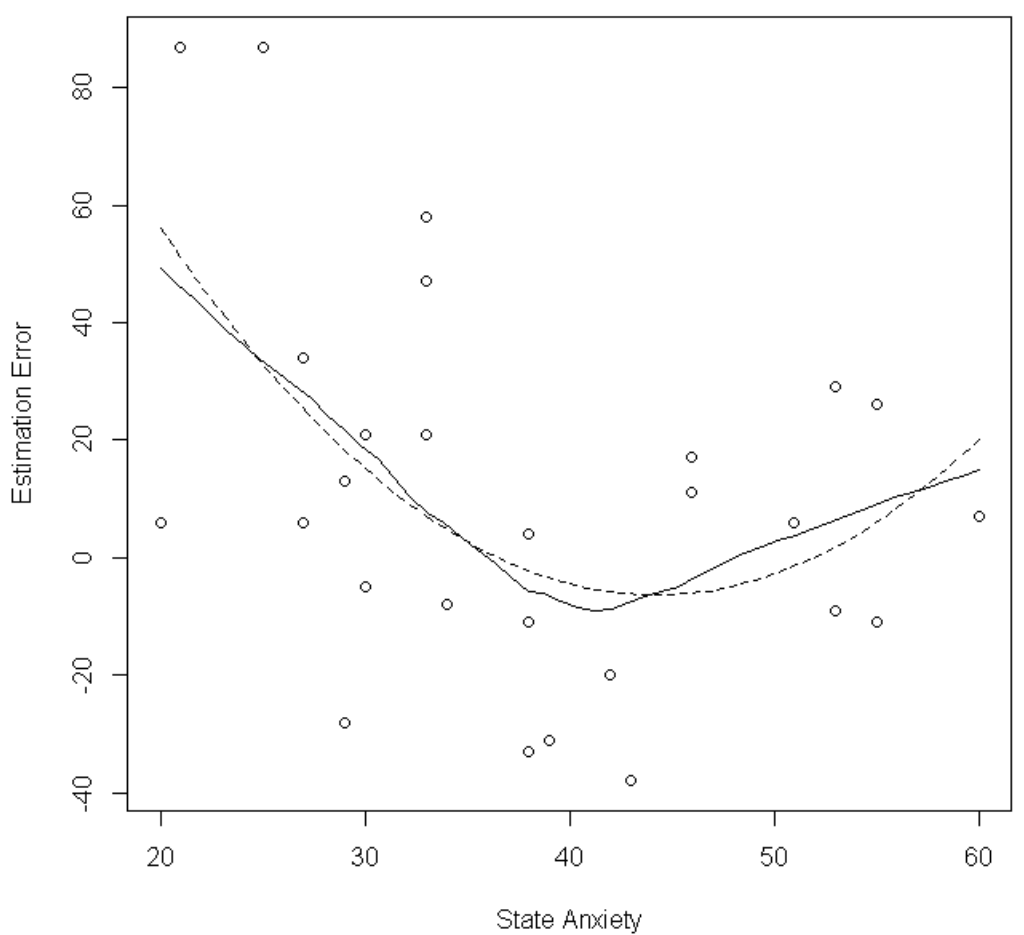

Figure 2. Curvilinear regression analysis delineating a substantial curvilinear trend between state-anxiety score and general time-estimation error.

\section{DISCUSSION}

It was predicted that higher state-anxiety scores would correspond with participants overestimating their assigned interval durations. The results of a t-test did not indicate that higher state-anxiety scores corresponded with an overestimation of interval durations. Though the hypothesis was not supported, the results of a multiple regression analysis did indicate that there was an effect for state-anxiety scores on participant-estimated time error. The curvilinear regression analysis was calculated to examine differences between participants with highest state-anxiety scores and participants with lowest state anxiety scores as a function of participant-estimated time error. When the data were analyzed to find if there were any effects for state-anxiety score and general time-estimation error, a significant negative correlation was found in the first analysis between those two variables. The discovered relationship appeared to be due to a very strong negative correlation in participants with anxiety scores less than 45 . The results of the quadratic curvilinear test agree with the results of the correlation test but go further by suggesting that participants whose state-anxiety scores were between 30 and 50 had the least amount of error when estimating their interval durations, compared to participants with both the lowest and the highest state-anxiety scores. While both the lowest and the highest state-anxiety scores were associated with more error, it should be noted that the participants with lowest state-anxiety scores had more timeestimation error than those with the highest state-anxiety scores.

The effects of anxiety on time perception reported here are bidirectionally consistent with previous work and strongly suggest that valenced emotional states, whether positive or negative, interfere with available attentional resources and distort time perception. However, the findings of the present study indicate that both low and high emotional arousal distorts the availability of attentional resources. These findings are consistent with the accumulator pacemaker models of time perception because emotional arousal disrupts attentional resources which complicate time estimation ability. While there was a curvilinear relationship between stateanxiety score and general time-estimation error, it is important to interpret this information with caution because it is uncertain 
why the highest and lowest state-anxiety scores had more time estimation error than mid-level state-anxiety scores. The data seems to indicate that in order to estimate time accurately a person has to be calm, but not so calm that they lose focus on the interval they are estimating, and perhaps a certain degree of anxiousness could be the result of prolonged focus, though this is more speculation than an empirically based assertion. The results of a linear trend analysis revealed a statistically significant positive linear relationship between interval duration and participant estimation error. Longer duration intervals were related to more participant-estimated error. The findings of this study are consistent with the findings of previous studies, even though other studies typically utilized shorter interval durations (e.g. one, three, seven, and 20 seconds). ${ }^{17,}{ }^{18}$ Researchers from past studies have reported that with longer interval durations there was more participant-estimated time error. ${ }^{17}$, 18 Perhaps longer interval durations are harder for the brain to process than shorter durations because of attentional resource limitations, though the exact cause of this phenomenon is still unknown.

Although there were statistically significant results attained in this study there were some limitations. A limitation of this study was the relatively small sample size. There were only 30 participants. For comparison, similar studies done in the past have involved as many as 100 participants. ${ }^{16}$ In addition to a small sample size, the researcher only recruited college students to participate, which significantly skewed the age demographic to represent primarily young adults. It should also be noted a few technical challenges could have compromised the validity of this study. During participant testing, the researcher was present in order to record participants' time estimates. After a few participants had completed their time estimations, they informed the researcher that sitting silently in the room while the researcher sat across from them was awkward. Since awkwardness is an unpleasant sensation, those negative emotions may have disrupted the participants' attentional resources, thus confounding their ability to estimate time. In order to avoid this issue in the future, it would be best if the researcher were absent from the room while the participants estimated their assigned time intervals. Perhaps a reaction time button could be pressed by participants and times could be recorded automatically in the absence of the researcher. In this manner, the researcher-participant proximity awkwardness would be eliminated. Because the researcher had to manually measure each participant's response using a stopwatch, the researcher was neither blind to who was participating in the study nor blind to which interval group a particular participant belonged in. Thus, the method by which the experiment was conducted could have influenced the findings, as the presence of the researcher in the room could have caused participants to become more relaxed or more anxious.

\section{FUTURE STUDIES}

Future modifications to the study could include the addition of distractions, such as having one group of participants engage in something they consider to be a fun or enjoyable activity (ex: playing a video game) and having the other group engage in something they consider a boring activity (ex: reading a chemistry textbook). These distractions could test the popular phrase "time flies by when you're having fun." However, it would be necessary to ask participants to disclose whether they actually found the activity enjoyable or boring as these can differ between participants. While only the emotional state of anxiety was examined in this study, psychological disorders that interfere with attentional resources and emotional arousal may also affect human time perception, such as attention-deficit hyperactivity disorder (ADHD) and depression. In ADHD, attentional resources are diminished, which may affect time perception. In fact, studies on ADHD and time perception have indicated statistically significant duration discrimination deficits in children with ADHD, in that children with ADHD are more likely to make discrimination errors. ${ }^{20}$

\section{CONCLUSION}

The present study demonstrated significant results for the effects of interval duration and state-anxiety on time perception. In a time-based society where human behavior is often governed by deadlines and time parameters, modern human existence is profoundly affected and measured by the clock. Even with the invention of watches and cell phones to inform humans minute by minute of the time, it is still a challenge to conform to the unchanging rigidity of clock time. Continued study of time perception will not only broaden scientific understanding of how and why human time perception differs from real time, but may also garner knowledge that may help humans make better use of their time and effectively improve their quality of life.

\section{ACKNOWLEDGEMENTS}

The author of this paper thanks Dr. Joan Piroch and Dr. William Kind for their mentorship in guiding the development of this paper. 


\section{REFERENCES}

1. Tanaka, H., Homma, K., \& Imamizu, H. (2012). Illusory reversal of causality between touch and vision has no effect on prism adaptation rate. Frontiers in Psychology, 3, 545.

2. Grondin, S., \& Rousseau, R. (1991). Judging the relative duration of multimodal short empty time intervals. Perception and Psychophysics, 49(3), 245-256.

3. Gibbon, J. (1977). Scalar expectancy theory and Weber's law in animal timing. Psychological review, 84(3), 279.

4. Fortin, C., \& Couture, E. (2002). Short-term memory and time estimation: Beyond the 2-second "critical" value. Canadian Journal of Experimental Psychology, 56(2), 120-127.

5. Wencil, E. B., Coslett, H. B., Aguirre, G. K., \& Chatterjee, A. (2010). Carving the clock at its component joints: neural bases for interval timing. Journal of neurophysiology, 104(1), 160-168.

6. Kojima, Y. \& Matsuda, F. (2000). Effects of attention and external stimuli on duration estimation under a prospective paradigm. Japanese Psychological Research, 42(3), 144-154.

7. Buhusi, C. V., \& Meck, W. H. (2005). What makes us tick? functional and neural mechanisms of interval timing. Nature Reviews Neuroscience, 6(10), 755-765.

8. Bush, G., Luu, P., \& Posner, M. I. (2000). Cognitive and emotional influences in anterior cingulate cortex Elsevier Ltd.

9. Carretie, L., Hinojosa, J.A., Martin-Loeches, M., Mercado, F., \& Tapia, M. (2004). Automatic attention to emotional stimuli: Neural correlates. Human Brain-Mapping, 22(4), 290-299.

10. Manuello, J., Vercelli, U., Nani, A., Costa, T., \& Cauda, F. (2016). Mindfulness meditation and consciousness: An integrative neuroscientific perspective. Consciousness and Cognition, 40, 67-78.

11. Droit-Volet, S., Fanget, M., \& Dambrun, M. (2015). Mindfulness meditation and relaxation training increases time sensitivity. Consciousness and Cognition, 31, 86-97.

12. Carrasco, M.C., \& Redolat, R., \& Simon, V.M. (1998). Effects of cigarette smoking on time estimation. Human Psychopharmacology, 13, 565-573.

13. Ashare, R. L., \& Kable, J. W. (2015). Sex Differences in Time Perception During Smoking Abstinence. Nicotine \& Tobacco Research, 17(4), 449-454.

14. Kelly, W. E. (2002). Anxiety and the prediction of task duration: A preliminary analysis. The Journal of Psychology, 136(1), 53-58.

15. Kelly, W. E., Johnson, J. L., \& Miller, M. J. (2003). Conscientiousness and the prediction of task duration. North American Journal of Psychology, 5(3), 443.

16. Kelly, W. E. (2004). College Students' Accuracy and Perceptions of Accuracy in Predicting the Duration of an Academic-Related Task. Individual Differences Research, 2(3), 225-230.

17. Koivula, N. (1995). Estimation of time: Effects of locus of control, mental arithmetic, and length of target interval. Personality, Individual Differences, and Intelligence, 20(1), 25-32.

18. Hancock, P.A., \& Raush, R. (2010). The effects of sex, age, and interval duration on the perception of time. Acta Psychologica, 133, 170-179.

19. Spielberger, C. D., Gorsuch, R.L., and Lushene. R.E. (1970). Manual for the State-Trait Anxiety Inventory. Palo Alto, CA: Consulting Psychologists Press.

20. Plummer, C., \& Humphrey, N. (2009). Time perception in children with ADHD: The effects of task modality and duration. Child Neuropsychology, 15, 147-162.

\section{ABOUT THE STUDENT AUTHOR}

The author completed this research as an undergraduate studying psychology at Coastal Carolina University during May of 2011. Currently, he's a graduate student at in the biological psychology program at Virginia Tech studying visual neuroscience and brain imaging.

\section{PRESS SUMMARY}

Time perception underlies our ability to understand the temporal order of causal events. While time perception is a fundamental of learning and human behavior, evidence suggests our perceptions of time are subject disturbances incurred by our emotional states. Thus, our emotional states, which can alter by a multitude of internal and external phenomena, to some degree dictate our perception of time and ultimately our ability to learn and interact with the world. 


\title{
On Closure Properties of Irrational and Transcendental Numbers under Addition and Multiplication
}

\author{
Shekh Mohammed Zahid ${ }^{*}$ and Dr. Prasanta Kumar Ray ${ }^{b}$ \\ anstitute of Mathematics and Applications, Bhubaneswar, Odisha, India \\ ${ }^{b}$ Veer Surendra Sai University of Technology, Burla, Odisha, India \\ *Student: shekhmohammedrabid@gmail.com \\ Mentor:prasanta@iiit-bh.ac.in
}

\begin{abstract}
In the article 'There are Truth and Beauty in Undergraduate Mathematics Research', the author posted a problem regarding the closure properties of irrational and transcendental numbers under addition and multiplication. In this study, we investigate the problem using elementary mathematical methods and provide a new approach to the closure properties of irrational numbers. Further, we also study the closure properties of transcendental numbers.
\end{abstract}

\section{KEYWORDS}

Irrational numbers; Transcendental numbers; Dedekind cuts; Algebraic numbers

\section{INTRODUCTION}

In article, ${ }^{1}$ a problem is posted to prove or disprove the following:

(a) The addition of two transcendental/irrational numbers is transcendental/ irrational

(b) The product of two transcendental /irrational numbers is transcendental/ irrational.

It is well known that the irrational numbers are those which cannot be represented as a ratio of integers. There are many popular irrational numbers like $\sqrt{2}$, Euler number $e$, Euler Mascheroni constant $\gamma$, golden ratio $\varphi$ and the irrational $\pi$. Algebraic numbers are those numbers, which are roots of polynomials with integral coefficients, otherwise are transcendental numbers. Commonly known examples of transcendental numbers are $e$ and $\pi$.

In this study, first, we account on the existence of irrational numbers and discuss the decimal representation of these numbers. In section 2 of this article, motivated from paper, ${ }^{2}$ we establish the closure property of irrational numbers in a new way by using the infinite non-repeating and non-recurring decimal representation of these numbers. In section 3, the construction of transcendental numbers by Cantor's argument is discussed. Further, the main result on transcendental numbers which states that "The sum and product of transcendental numbers are not always transcendental, moreover at least one of the product or sum is transcendental," is proved.

\section{CLOSURE PROPERTIES OF IRRATIONAL NUMBERS}

It is quite reasonable to understand, how mathematicians came to know about the existence of irrational numbers. The first proof of the existence of irrational numbers is usually attributed to a Pythagorean who observed that in a right-angled triangle, with height and base 1 unit gives the hypotenuse side $\sqrt{2}$ unit. The more rigorous proof of construction of irrational numbers is given by mathematician Richard Dedekind which is popularly known as Dedekind cuts. ${ }^{3}$

The following are some known preliminary results involving irrational numbers.

LEMMA 1. The decimal part of irrational numbers is non-repeating and non-recurring.

PROOF. Consider a real number $\delta$ with repeating decimal

$$
\delta=a_{0} \cdot a_{1} a_{1} a_{1} \ldots
$$

Equation 1. 
In order to prove that $\delta$ is a rational number, multiplying Equation 1 by 10 and subtract it from Equation 1, we get

$$
\delta=\frac{a_{0}\left(a_{1}-1\right)}{9}
$$

which is clearly a rational number. In general, let $\varepsilon$ be a real number with repeating digits having period $n$. Then,

$$
\varepsilon=b_{0} . b_{1} b_{2} b_{3} \ldots b_{n} b_{1} b_{2} b_{3} \ldots b_{n} b_{1} b_{2} b_{3} \ldots
$$

Equation 2.

Multiplying Equation 2 by $10^{\mathrm{n}}$ and subtracting it from Equation 2, we get

$$
\varepsilon=\frac{b_{0}\left(b_{1} b_{2} b_{3} \ldots b_{n}-1\right)}{\left(10^{n}-1\right)}
$$

which is also a rational number. This completes the proof.

We now investigate that why sum and product of two irrational numbers are rational or irrational. Though it is quite trivial by considering some examples like "Sum of $(\sqrt{2}+1)$ and $(3-\sqrt{2})$ and product of $(\sqrt{2}-1)$ and $(\sqrt{2}+$ 1) are rational" and "Sum of $\sqrt{2}$ and $\sqrt{3}$ and product of $\sqrt{2}$ and $\sqrt{3}$ are irrational". But, we take a close look what is actually happening in these operations. For example, summing two irrational numbers $(\sqrt{2}+1)$ and $(3-\sqrt{2})$, one observes that the approximate sum of their decimal parts gives, $0.41421356237+0.58578643763=1.0$ and terminating the fractional part, giving the overall sum is nothing but an integer. Now considering the sum of $\sqrt{2}$ and $\sqrt{3}$, in which the approximate sum fractional part gives 1.14626436994. In this example, the approximate sum of the fractional part is non-terminating and non-recurring even when number of decimal part increased, this is a property of irrational numbers.

From above two examples, we observe that the decimal part decides the rationality and irrationality of a number. We use this idea in order to investigate the problem posted.

The following identity is useful while proving the subsequent results.

PROPOSITION 1. Any irrational number $\alpha$ can be written as,

$$
\alpha=[\alpha]+\{\alpha\}
$$

where [ ] denotes the integral part and \{\} denotes the fractional part. Moreover, $\{\alpha\}=\lim _{n \rightarrow \infty} \sum_{i=1}^{n} \frac{a_{i}}{10^{i}}$,

where $a_{i} \in 0,1,2 \ldots 9$.

The following result shows that the sum of two irrational numbers is not always irrational.

THEOREM 1. The sum of two irrational numbers is not always irrational. In fact, the decimal part of the sum decides rationality or irrationality.

PROOF. Let $\gamma$ and $\beta$ are two irrational numbers. By virtue of Proposition 1,

$$
\gamma=[\gamma]+\{\gamma\} \text { and } \beta=[\beta]+\{\beta\}
$$

Moreover, $\{\gamma\}=\lim _{n \rightarrow \infty} \sum_{i=1}^{n} \frac{c_{i}}{10^{i}}$ and $\{\beta\}=\lim _{n \rightarrow \infty} \sum_{i=1}^{n} \frac{b_{i}}{10^{i}}$, where $c_{i}$ and $b_{i}$ are one of the digits 0 to 9. This implies that

$$
\gamma+\beta=[\gamma]+\{\gamma\}+[\beta]+\{\beta\}
$$

Since, $[\gamma]$ and $[\beta]$ are integers then, their sum is also an integer. Now we take a look at sum of fractional parts, i.e.

$$
\{\beta\}+\{\gamma\}=\lim _{n \rightarrow \infty} \sum_{i=1}^{n} \frac{c_{i}+b_{i}}{10^{i}}
$$


The possible cases are

CASE 1. If $\lim _{n \rightarrow \infty} \sum_{i=1}^{n} \frac{c_{i}+b_{i}}{10^{i}}=k_{0} . k_{1} k_{1} k_{1} \ldots$, where $k_{i}$ is one of the digits of 0 to 9 . Then, $\gamma+\beta$ is rational number due to repeating property of decimal part.

CASE 2. If $\lim _{n \rightarrow \infty} \sum_{i=1}^{n} \frac{c_{i}+b_{i}}{10^{i}}=k_{0} . k_{1} k_{2} k_{3} \ldots k_{n} k_{1} k_{2} k_{3} \ldots k_{n} \ldots k_{1} k_{2} \ldots$, where $k_{i}$ is one of the digits of 0 to 9 .Then, $\gamma+\beta$ is rational number due to recurring property of decimal part.

CASE 3. If $\lim _{n \rightarrow \infty} \sum_{i=1}^{n} \frac{c_{i}+b_{i}}{10^{i}}=k_{0} . k_{1} k_{2} k_{3} k_{4} k_{5} \ldots$, where $k_{i}$ is one of the digits of 0 to 9 . Then, $\gamma+\beta$ is irrational number due to non-repeating and non-recurring property of decimal part.

Similarly, the product of two irrational numbers may not be irrational. The following result shows this fact.

THEOREM 2. The product of two irrational numbers is not always irrational. In fact, the decimal part of the product operation decides rationality or irrationality.

PROOF. Let $\gamma$ and $\beta$ are two irrational numbers. By virtue of Proposition 1,

$$
\begin{gathered}
\gamma \times \beta=([\gamma]+\{\gamma\}) \times([\beta]+\{\beta\}) \\
=[\gamma] \times[\beta]+[\gamma] \times\{\beta\}+\{\gamma\} \times[\beta]+\{\gamma\} \times\{\beta\} .
\end{gathered}
$$

The affecting property of irrationality is decimal part, now we concentrate at decimal part of product of $\gamma$ and $\beta$, since $[\gamma] \times[\beta]$ is integer.

Since, $[\gamma] \times\{\beta\}+\{\gamma\} \times[\beta]+\{\gamma\} \times\{\beta\}$ is $\lim _{n \rightarrow \infty} \sum_{i, j=1}^{n} \frac{[\beta] c_{i}+[\gamma] b_{i}+c_{i} b_{j}}{10^{i}}$, possible cases are

CASE 1. If $\lim _{n \rightarrow \infty} \sum_{i, j=1}^{n} \frac{[\beta] c_{i}+[\gamma] b_{i}+c_{i} b_{j}}{10^{i}}=k_{0} . k_{1} k_{1} k_{1} \ldots$, where $k_{i}$ is one of the digits of 0 to 9 . Then, $\gamma \times \beta$ is rational number due to repeating property of decimal part.

CASE 2. If $\lim _{n \rightarrow \infty} \sum_{i, j=1}^{n} \frac{[\beta] c_{i}+[\gamma] b_{i}+c_{i} b_{j}}{10^{i}}=k_{0} . k_{1} k_{2} k_{3} \ldots k_{n} k_{1} k_{2} k_{3} \ldots k_{n} k_{1} k_{2} \ldots$, where $k_{i}$ is one of the digits of 0 to 9 . Then, $\gamma \times \beta$ is rational number due to recurring property of decimal part.

CASE 3. If $\lim _{n \rightarrow \infty} \sum_{i, j=1}^{n} \frac{[\beta] c_{i}+[\gamma] b_{i}+c_{i} b_{j}}{10^{i}}=k_{0} . k_{1} k_{2} k_{3} k_{4} k_{5} \ldots$ where $k_{i}$ is one of the digits of 0 to 9 . Then, $\gamma \times \beta$ is irrational number due to non-repeating and non-recurring property of decimal part.

\section{CLOSURE PROPERTIES OF TRANSCENDENTAL NUMBERS}

Joseph Liouville first proved the existence of transcendental numbers. He gave the first decimal examples such as the Liouville constant i.e. $\sum_{k=1}^{\infty} 10^{-k}$. The construction of transcendental numbers also comes to know from Cantor arguments. By definition, a set A is said to be countable if there exists a one to one correspondence between A to $\mathbf{N}$, where $\mathbf{N}$ is set of natural numbers. It is well known that the set of real numbers are uncountable by Cantor diagonalisation argument. Since, Cantor proved, in the set of real numbers, the numbers which are roots of polynomials with integral coefficients i.e. the set of algebraic numbers is countable, so there also exist numbers which are not roots of polynomial, uncountable i.e. the set of transcendental numbers. ${ }^{4}$

The following result shows that for any two transcendental numbers, at least one of sum or product of these numbers is transcendental.

THEOREM 3. If $\omega$ and $\varphi$ are two transcendental numbers, then at least one of $\omega+\varphi$ or $\omega \times \varphi$ is transcendental.

PROOF. We use method of contradiction to prove this result. Consider two transcendental numbers $\omega$ and $\varphi$. Suppose if possible $\omega+\varphi$ and $\omega \times \varphi$ both are algebraic numbers. 
Then,

$$
\omega+\varphi=a_{0} \text { and } \omega \times \varphi=a_{1},
$$

Equation 3.

where $a_{0}$ and $a_{1}$ are algebraic. Simplification of Equation 3 gives a polynomial with algebraic coefficients, $\omega^{2}+$ $a_{1} \omega-a_{0}=0$. Since, polynomial with algebraic coefficients always gives algebraic roots, ${ }^{5}$ which contradicts the fact that $\omega$ is a transcendental root. This completes the proof.

\section{CONCLUSION}

In this article, we see the existence and construction of irrational and transcendental numbers. Followed by it, by using the concepts of decimal representation, we get the conclusion that the sum and product of irrational and transcendental numbers are not always irrational or transcendental.

\section{ACKNOWLEDGMENTS}

I truly thank my siblings, Kuhu and Aadil for supporting me during this work. I am very grateful to Dr. Dele Oluwade for proposing this problem to me and Dr. Prasanta Kumar Ray for help with this paper.

\section{REFERENCES}

1. Oluwade D. (2004), There is Truth and Beauty in Undergraduate Mathematics Research, American Journal of Undergraduate Research, 2 (4), pp- 5-6.

2. Zahid S.M., Ray P.K. (2015), Approximation of Euler Number Using Gamma Function, American Journal of Undergraduate Research, 12 (3), pp- 45-52, ISSN-2375-8732.

3. Rudin.W (2013), Principles of Mathematical Analysis, 3 ${ }^{\text {rd }}$ Edition, pp- 1-4, McGraw Hill Education Private Limited, India.

4. Niven I. (1961), Numbers: Rational and Irrational, $2^{\text {nd }}$ printing, pp- 122-128, Random House, New York.

5. Artin.M (2006), Algebra, Indian Edition, pp-527-531, Prentice-Hall of India Private Limited, India.

\section{ABOUT THE STUDENT AUTHOR}

Shekh Mohammed Zahid is a first-year undergraduate student of BSc(Hons) in Mathematics and Computing in the Institute of Mathematics and Applications, Odisha, India. His research interest includes number theory and theoretical physics. He likes to play chess.

\section{PRESS SUMMARY}

In this article, we provide a solution to a problem posted on American Journal of Undergraduate Research (AJUR) regarding closure properties of irrational and transcendental numbers under the operation of addition and multiplication. Though the problem already has a solution, in this article, we provide a new approach to solving it. 


\title{
Peaked T-Waves and Potassium: The Reliability of Peaked T-Waves as Indicators of Hyperkalemia in End-Stage Renal Patients Undergoing Hemodialysis
}

\author{
Ryan D. Pappal*a, Ilse M. Espina ${ }^{b}$ Zubaid Rafique \\ ${ }^{a}$ Rice University, Houston, TX \\ ${ }^{b}$ Section of Emergency Medicine, Baylor College of Medicine, Houston, TX \\ Student:pappal.ryan@gmail.com*,pappal.ryan@rice.edu \\ Mentor:zubaidrafique@gmail.com*,rafique@bcm.edu
}

\begin{abstract}
While peaked T-waves have traditionally been associated with hyperkalemia cases, previous studies have called their reliability for hyperkalemia diagnosis into question. This study examined 89 ESRD patients totaling to 736 patient visits. Linear regressions and receiver operating characteristic (ROC) analyses were utilized to assess the reliability of peaked T-waves as a diagnostic criterion for hyperkalemia. Poor correlations between T/R ratio (leads V2-V4) and serum potassium were found, $\mathrm{r}=0.23$, 0.17 , and 0.17 , $p<0.001$. $\mathrm{R}^{2}$ values ranged from 0.03 to 0.05 . ROC analysis found poor sensitivity and specificity of $\mathrm{T} / \mathrm{R}$ as a predictor of hyperkalemia (area under the curve: $0.61-0.66$ ), and the $T / R \geq 0.75$ threshold yielded a sensitivity of 0.83 with a specificity of 0.40 when predicting hyperkalemia (serum potassium $>5.5 \mathrm{mEq} / \mathrm{L}$ ). We conclude that the peaked T-wave is a poor indicator for the early identification of hyperkalemia in ESRD patients presenting to the emergency department.
\end{abstract}

\section{KEYWORDS}

Hyperkalemia; Electrocardiography; End-stage Renal Disease; Hemodialysis; Arrhythmias, Potassium; Sensitivity and Specificity; ROC Curve

\section{INTRODUCTION}

Chronic kidney disease (CKD) is a common disorder that predisposes patients to dangerous metabolic conditions. ${ }^{1}$ Hypertension and diabetes mellitus are known risk factors for the development of CKD. The primary causes of renal failure are diabetic glomerulosclerosis and hypertensive nephrosclerosis, although chronic kidney disease has been known to precipitate from infections and exposure to illicit drugs and toxins, particularly in developing countries. ${ }^{2}$ Kidney dysfunction that progresses to the point that regular dialysis or a kidney transplant is required is defined as end-stage renal disease (ESRD). ${ }^{2}$

Hyperkalemia is defined as a serum potassium greater than $5.5 \mathrm{mEq} / \mathrm{L}$ and is a life-threatening electrolyte imbalance frequently observed in ESRD patients. ${ }^{1,3}$ In these patients, impaired glomerular filtration rate combined with the extracellular shift of potassium caused by acidosis impair the ability to excrete potassium. ${ }^{1,4}$ Hemodialysis is a common means for ESRD patients to maintain healthy electrolyte levels; however, at least one study has shown that the long hiatus from dialysis experienced during the weekend increases the risk of electrolyte imbalance formation, particularly hyperkalemia, and subsequently the risk of sudden cardiac death. ${ }^{3}$ Only modest changes in serum potassium in patients with existing cardiovascular or renal disease can lead to increased risk of hospitalization or death..$^{1,5}$

Hyperkalemia induces a wide range of vague symptoms, particularly muscle spasms, nausea, and vomiting. ${ }^{6}$ Additionally, it works through a number of mechanisms to impair myocardial electrical conduction. Mild hyperkalemia, serum potassium between 5.5 $6.5 \mathrm{mEq} / \mathrm{L},{ }^{7}$ inhibits voltage-gated sodium channels by reducing the resting membrane potential. The subsequent decrease in $\mathrm{V}_{\text {max }}$ of these sodium channels blunts the rate of depolarization during phase 0 of the action potential, causing P-wave and QRS duration prolongation. ${ }^{7}$ When serum potassium rises above $6.5 \mathrm{mEq} / \mathrm{L},{ }^{7} \mathrm{IKr}$-mediated potassium currents increase due to heightened channel sensitivity from high extracellular potassium. Subsequent membrane repolarization during phase 3 of the myocardial action potential occurs more rapidly and thus with greater magnitude, manifesting as short-duration, peaked T-waves on ECG. ${ }^{7}$ Figure 1 shows a hyperkalemic patient's ECG with peaked T-waves and a prolonged QRS interval (A), as well as one from a different hyperkalemic patient that has normal T-waves and a standard QRS interval (B). As serum potassium progresses to well above $8.0 \mathrm{mEq} / \mathrm{L}$, the QRS complex widens substantially and can fuse with the T-wave, forming a classic sine-wave ECG pattern that is indicative of impending cardiac arrest from ventricular fibrillation or asystole. ${ }^{6,8,9}$ 


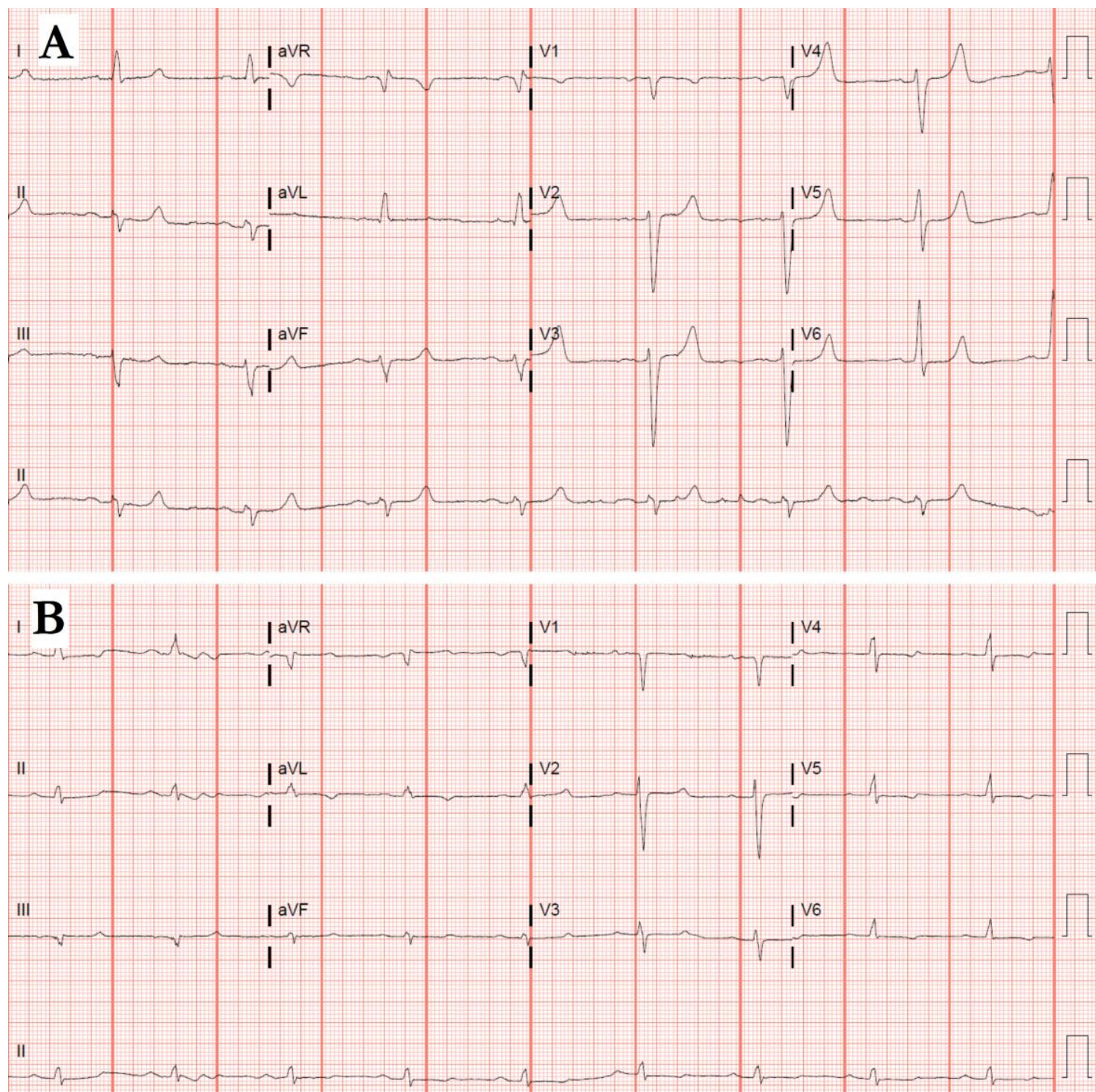

Figure 1. ECGs from ESRD patients in severe hyperkalemia. A) Patient with a serum potassium of $7.3 \mathrm{mEq} / \mathrm{L}$. ECG shows peaked Twaves in V2, V3, V4 and QRS prolongation (QRSD = $138 \mathrm{~ms})$. B) Patient with a serum potassium of $7.5 \mathrm{mEq} / \mathrm{L}$. ECG has non-peaked Twaves and normal QRS duration (QRSD = $104 \mathrm{~ms})$.

Since hyperkalemia may cause serious cardiac complications, rapid identification and treatment are essential to improving outcomes. Clinical guidelines suggest the utilization of 12-lead electrocardiograms (ECGs) to detect hyperkalemia alongside blood tests. ${ }^{9}$ However, the status of peaked T-waves as indicators of hyperkalemia has been called into question in some recent retrospective studies. A review of 127 instances of diagnosed hyperkalemia showed that only $46 \%$ had any ECG changes suggestive of hyperkalemia; furthermore, the severity of hyperkalemia had no significant correlation with the presence of classic ECG signs. ${ }^{10} \mathrm{~A}$ study of 74 hemodialysis patients reported no significant differences in $\mathrm{T}$-wave amplitudes and $\mathrm{T} / \mathrm{R}$ ratios in the precordial leads between normal and elevated serum potassium level.11 In another review of 90 hyperkalemic patients, only 24 were noted to have T-wave abnormalities, with 21 of those noted as non-specific changes by reading cardiologists; furthermore, only 47 of the 90 patients had any ECG changes at all. ${ }^{6}$

The diagnostic difficulties caused by the unreliability of classic hyperkalemic ECG changes are exacerbated by the tendency of hyperkalemia to cause other, non-specific cardiac abnormalities. Severe hyperkalemia can also manifest in heart blocks, including fascicular blocks, bundle branch blocks, and complete heart block. ${ }^{13}$ The effect of hyperkalemia on ventricular repolarization has been known to cause widespread ST-elevation mimicking acute myocardial infarction patterns. ${ }^{6,13}$ Thus, even when hyperkalemia is manifesting in ECG changes, a clinician must be ready to screen out extraneous changes that suggest other dangerous 
conditions before chiefly suspecting hyperkalemia. Thus, if peaked T-waves are to be utilized as a diagnostic criterion, favorable sensitivity and specificity for ECG-based parameters must be established.

Our study examines ECG and electrolyte data from 89 ESRD patients who have undergone hemodialysis for at least three months. We examined whether or not peaked T-waves are reliable indicators of hyperkalemia in ESRD patients.

\section{METHODS AND PROCEDURES}

This study was a retrospective chart review of a cohort of dialysis patients at an inner city county hospital in a major metropolitan city. Eighty-nine ESRD patients were included in the review who visited Ben Taub General Hospital between June 2012 and June 2014. The study was approved by the Baylor College of Medicine institutional review board. Patients were included if they were older than 18 years of age and received dialysis for more than 3 months. Patients were excluded if they did not have a readable ECG tracing or a recorded basic metabolic panel associated with their tracing.

A total of 89 patients (49 male) were enrolled and 736 data points were recorded. Each data point was a unique patient-visit for emergent dialysis, consisting of one ECG and corresponding electrolyte levels. ECGs used were from intake before any treatment was administered. Peaked T-wave definition of $\mathrm{T} / \mathrm{R}$ ratio $\geq 0.75$ was used to calculate its prevalence. T-wave and $\mathrm{R}$-wave amplitudes were measured by hand on printed ECGs for leads V2, V3, and V4. ECG data was measured without knowledge of serum potassium level.

Data points were excluded if the electronic interpretation of the ECGs included left ventricular hypertrophy, right or left bundle branch block, or artificial pacemaker spikes. Furthermore, ECGs with a wandering baseline or other characteristic that prevented a reliable measurement of $\mathrm{T} / \mathrm{R}$ were excluded. Unreadable $\mathrm{R}$-wave amplitudes due to low voltage were also excluded. To assess our hypothesis that peaked $\mathrm{T}$-waves are poor clinical indicators of hyperkalemia, we assessed for an association between $\mathrm{T} / \mathrm{R}$ ratio and serum potassium level with linear regression models in leads V2, V3, and V4, and an interaction with calcium level was assessed for. Then, to determine the how well peaked T-wave performed as a diagnostic criterion for hyperkalemia, we ran receiver operating characteristic analyses. Custom statistical packages (PSPP and R) and plotting software (Microsoft Excel) were utilized for analysis. The specialized R package ROCR was utilized for receiver operating characteristic visualization and analysis. ${ }^{12}$

\begin{tabular}{ll}
\hline Variable & Value \\
\hline Patients & 89 \\
Mean Age \pm S.D. & $46 \pm 15$ \\
Sex & \\
Male & 49 \\
Female & 40 \\
Race (Ethnicity) & 85 \\
Hispanic / Latino & 2 \\
Black / African American & 2 \\
Middle Eastern & 736 \\
Patient-Visits & 547 \\
Hyperkalemia & $358-65.4 \%$ \\
Peaked T-Wave & $189-34.6 \%$ \\
Normal T-Wave & 189 \\
Normokalemia & $80-42.3 \%$ \\
Peaked T-Wave & $109-57.7 \%$ \\
$\quad$ Normal T-Wave & \\
Mean Electrolyte Levels \pm S.D. & $5.91 \pm 0.90$ \\
Potassium & $107.1 \pm 5.39$ \\
Chloride & $93.8 \pm 26.8$ \\
Blood Urea Nitrogen & $13.2 \pm 6.03$ \\
Creatinine & $0.96 \pm 0.15$ \\
Ionized Calcium & \\
\hline Table 1. Demographics, prevalence, and descriptive statistics. \\
aHyperkalemia is defined as serum potassium $\geq 5.5 \mathrm{mEq} / \mathrm{L}$ \\
bPeaked T-wave is defined as T/R ratio $\geq 0.75$ in V2, V3, or V4 \\
$\quad$
\end{tabular}




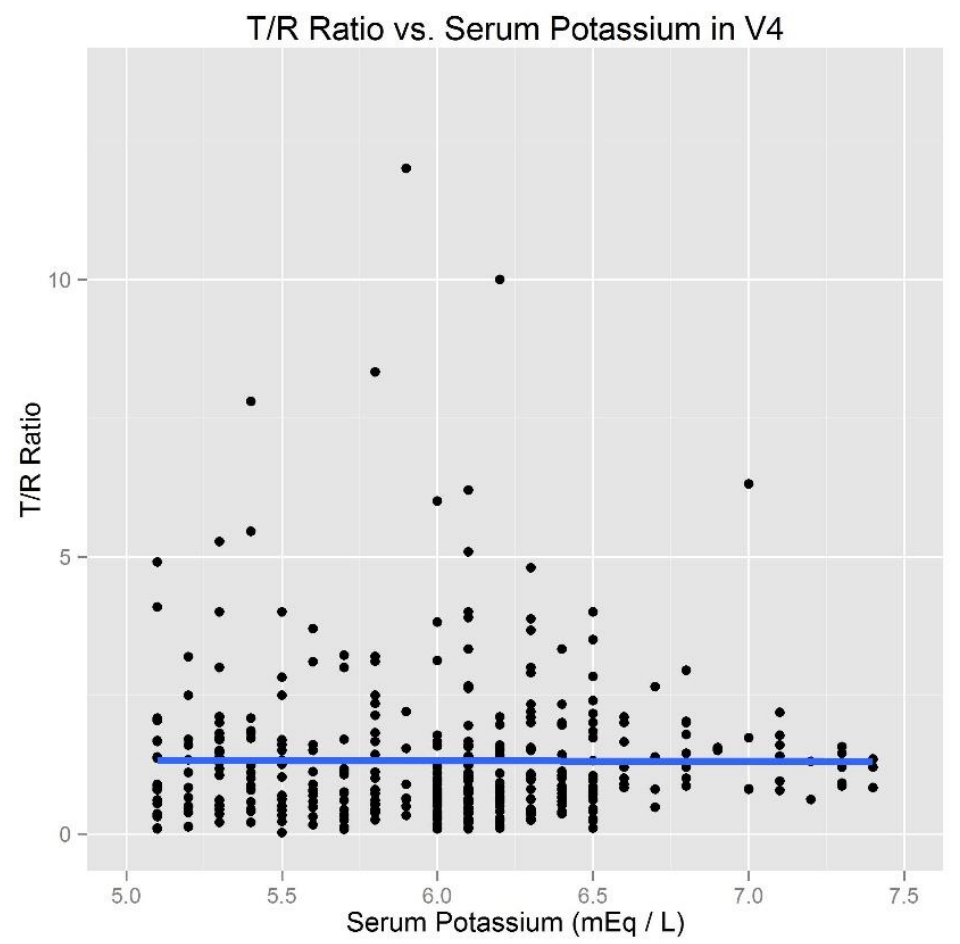

Figure 2. Linear regression model in V4. Graph shows T-wave to R-wave amplitude ratio vs. serum potassium scatter plot with a regression line. Analysis revealed a poor correlation consistent with that in leads V2 and V3, $\mathrm{r}=0.17, p<0.001$. Only $3 \%$ of the variation in $\mathrm{T} / \mathrm{R}$ ratio data could be explained by the linear model, $\mathrm{R}^{2}=0.03$.

\section{RESULTS}

The mean potassium level $\left(\left[\mathrm{K}^{+}\right]\right)$for the data set was $5.9( \pm 0.9) \mathrm{mEq} / \mathrm{L}$, and $\left[\mathrm{K}^{+}\right]$ranged from $3.1-8.4 \mathrm{mEq} / \mathrm{L}$. There were 49 males (55\%) and 40 females (45\%) in the cohort. The sample overwhelmingly identified as Hispanic/Latino (96\%). The mean age of the participants was 46.6 ( \pm 15.1 ) years. The prevalence of acute hyperkalemia was $74 \%$. Using the definition of a peaked $\mathrm{T}$ wave as $\mathrm{T} / \mathrm{R} \geq 0.75$, the prevalence of peaked $\mathrm{T}$-wave appearance in any of $\mathrm{V} 2, \mathrm{~V} 3$, or V4 was $59.5 \%$. Specific prevalence of peaked T-waves in V2, V3, and V4 were $48.0 \%, 39.0 \%$, and $21.1 \%$ respectively. Demographics of participants and descriptive statistics for collected data is summarized in Table 1.

\begin{tabular}{lll}
\hline Lead & Correlation Coefficient $(\mathbf{r})$ & Adjusted $\mathbf{R}^{\mathbf{2}}$ \\
\hline V2 & $0.23^{*}$ & 0.05 \\
V3 & $0.17^{*}$ & 0.03 \\
V4 & $0.17^{*}$ & 0.03 \\
\hline
\end{tabular}

Table 2. Statistical correlation of $\mathbf{T} / \mathbf{R}$ ratio vs. serum potassium, by lead. A multiple regression analysis on T-wave to R-wave amplitude ratios vs. serum potassium levels revealed poor correlations. Correlation coefficients ranged from $0.17-0.24$, showing weak effects of serum potassium on $\mathrm{T} / \mathrm{R}$ ratio. All adjusted $\mathrm{R}^{2}$ values are at or under 0.05 , indicating that no more than $5 \%$ of the variability in $\mathrm{T}$-wave amplitudes or $\mathrm{T} / \mathrm{R}$ ratios could be explained by serum potassium levels. ${ }^{*} p<0.001$

Linear correlation data is summarized in Table 2. Regression analysis of T/R ratio vs. serum potassium for V2, V3, and V4, yielded poor correlations, with coefficients of $\mathrm{r}=0.23, \mathrm{r}=0.17$, and $\mathrm{r}=0.17$, respectively. While all correlations were statistically significant, $p<0.001$, variance accounted for by these models was limited, with $\mathrm{R}^{2}$ values of $0.05,0.03$, and 0.03 , respectively. No more than $5 \%$ of the variance in $\mathrm{T} / \mathrm{R}$ ratio was accounted for by the linear model with serum potassium. Scatter plot showing $\mathrm{T} / \mathrm{R}$ ratio vs. serum potassium for V4 is shown in Figure 2. The linear partial correlation was run while controlling for serum calcium. No changes in correlation significance or effect size were observed (data not shown). 


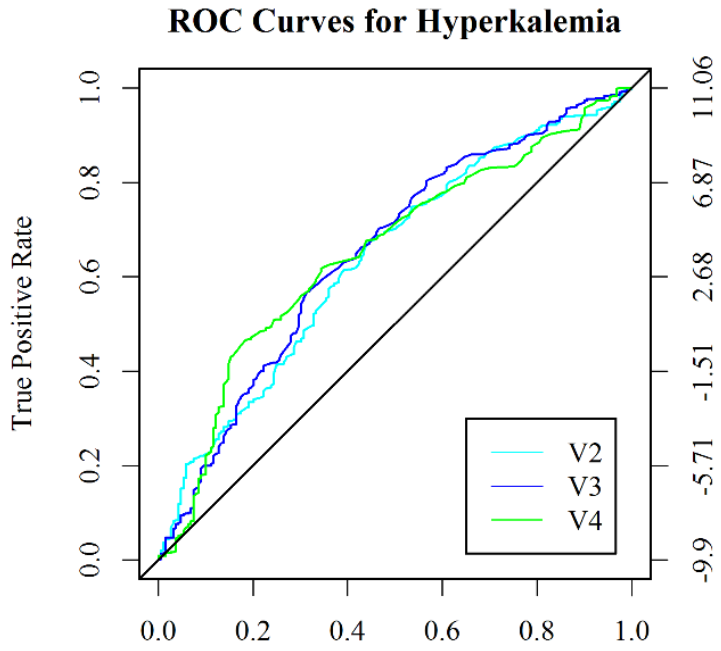

False Positive Rate
ROC Curves for Severe Hyperkalemia

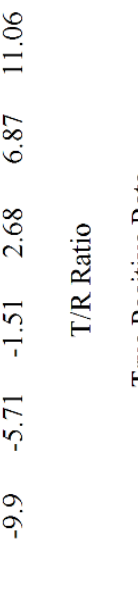

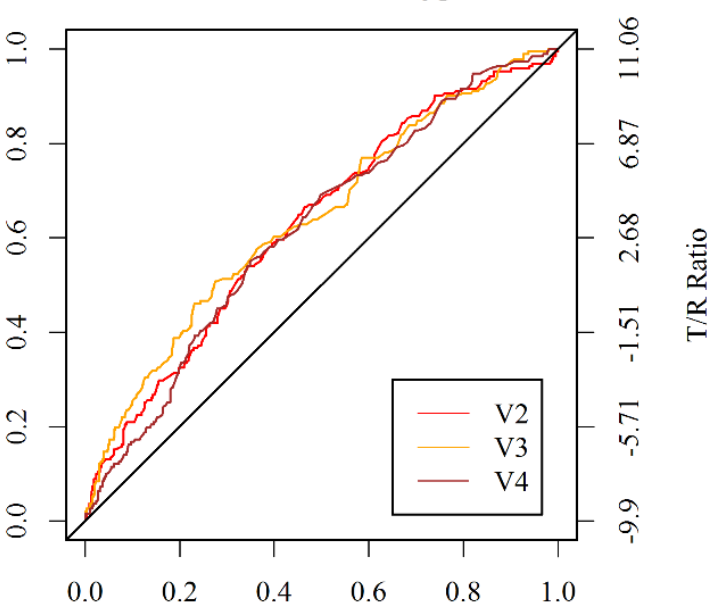

Figure 3. ROC curve analysis. Left-hand graph shows ROC curves for $\mathrm{T} / \mathrm{R}$ ratio as a diagnostic criterion for hyperkalemia (serum potassium $\geq 5.5 \mathrm{mEq} / \mathrm{L}$ ). Right-hand graph shows ROC curves for $\mathrm{T} / \mathrm{R}$ ratio as a diagnostic criterion for severe hyperkalemia (serum potassium $\geq 6.5 \mathrm{mEq} / \mathrm{L}$ ). The area under the curve (AUC) was calculated for each curve. All six ROC curves had AUCs significantly higher than the diagonal reference line with AUC $=0.5, p<0.001$. AUCs for the curves ranged from 0.61 to 0.66 .

Sensitivity and specificity were calculated for the peaked $T$-wave cutoff of $T / R \geq 0.75$. For hyperkalemia $\left(\left[\mathrm{K}^{+}\right] \geq 5.5\right.$ $\mathrm{mEq} / \mathrm{L})$, sensitivity and specificity were 0.83 and 0.40 , respectively. For severe hyperkalemia $\left(\left[\mathrm{K}^{+}\right] \geq 6.5 \mathrm{mEq} / \mathrm{L}\right)$, sensitivity and specificity were 0.88 and 0.27 , respectively. Receiver operating characteristic (ROC) analysis was utilized with $\mathrm{T} / \mathrm{R}$ ratio as a predictor of hyperkalemia (serum potassium $\geq 5.5 \mathrm{mEq} / \mathrm{L}$ ) and severe hyperkalemia (serum potassium $\geq 6.5 \mathrm{mEq} / \mathrm{L}$ ). Optimal $\mathrm{T} / \mathrm{R}$ ratio predictive thresholds were calculated by finding the maximum summed square of sensitivity and specificity. The area under the curve (AUC) was computed. The ROC curves are presented in Figure 3. Results of the analysis are summarized in Table 3. Optimal $\mathrm{T} / \mathrm{R}$ ratio predictive thresholds for hyperkalemia in V2, V3, and V4 were $0.88,0.81$, and 0.42 , respectively. Sensitivities (and specificities) for these thresholds were 0.61 (0.61), 0.59 (0.66), and 0.62 (0.66), respectively. Computed AUCs of the $\mathrm{ROC}$ curves for $\mathrm{T} / \mathrm{R}$ ratio for both hyperkalemia and severe hyperkalemia ranged from 0.60 to $0.66, p<0.001$.

\begin{tabular}{|c|c|c|c|c|c|}
\hline & Lead & Optimal T/R Threshold & Optimal Sensitivity & Optimal Specificity & $\begin{array}{l}\text { Area Under ROC Curve } \\
(95 \% \mathrm{CI})\end{array}$ \\
\hline \multirow{3}{*}{ 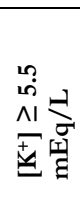 } & V2 & 0.88 & 0.614 & 0.608 & $\begin{array}{l}0.638^{*} \\
(0.592-0.684)\end{array}$ \\
\hline & V3 & 0.81 & 0.592 & 0.656 & $\begin{array}{l}0.651^{*} \\
(0.605-0.697)\end{array}$ \\
\hline & V4 & 0.42 & 0.618 & 0.656 & $\begin{array}{l}0.652 * \\
(0.608-0.697)\end{array}$ \\
\hline \multirow{3}{*}{ 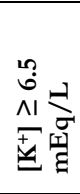 } & $\mathrm{V} 2$ & 1.11 & 0.586 & 0.606 & $\begin{array}{l}0.629 * \\
(0.584-0.675)\end{array}$ \\
\hline & V3 & $\begin{array}{l}0.99 \\
\end{array}$ & 0.576 & 0.637 & $\begin{array}{l}0.640^{*} \\
(0.594-0.686) \\
\end{array}$ \\
\hline & V4 & 0.61 & 0.550 & 0.651 & $\begin{array}{l}0.619 * \\
(0.573-0.664)\end{array}$ \\
\hline
\end{tabular}

Table 3. Summary of receiver operating characteristic analysis on $\mathbf{T} / \mathbf{R}$ ratio. Receiver operating characteristic (ROC) analysis was run on $\mathrm{T} / \mathrm{R}$ ratio for predictive performance of hyperkalemia (serum potassium $\geq 5.5 \mathrm{mEq} / \mathrm{L}$ ) and severe hyperkalemia (serum potassium $\geq 6.5 \mathrm{mEq} / \mathrm{L}$ ). An optimal T/R ratio threshold was determined for each lead by optimizing true positive rate while minimizing false positive rate. These thresholds are reported above with the corresponding sensitivity and specificity values. The area under each ROC curve was computed and revealed poor criterion performance, with all calculated areas between 0.61 and $0.66 .{ }^{*} p<0.001$ 


\section{DISCUSSION}

Our study examined 89 end-stage renal disease patients receiving emergent dialysis for at least three months. $\mathrm{T} / \mathrm{R}$ ratio has statistically significant but clinically poor correlation with serum potassium. The optimal correlation was noted in lead V2, where still only $5 \%$ of the variation in $\mathrm{T} / \mathrm{R}$ ratio was accounted for by serum potassium. Furthermore, receiver operating characteristic (ROC) analysis on $\mathrm{T} / \mathrm{R}$ ratio in $\mathrm{V} 2, \mathrm{~V} 3$, and $\mathrm{V} 4$ as predictive of hyperkalemia $\left(\left[\mathrm{K}^{+}\right] \geq 5.5 \mathrm{mEq} / \mathrm{L}\right)$ and severe hyperkalemia $\left(\left[\mathrm{K}^{+}\right]\right.$ $\geq 6.5 \mathrm{mEq} / \mathrm{L}$ ) indicated poor diagnostic performance. Optimal thresholds calculated by greatest summed square of sensitivity and specificity also yielded poor performance, with sensitivities ranging from 0.55 to 0.62 , and specificities ranging from 0.61 to 0.66 . We conclude that $\mathrm{T} / \mathrm{R}$ ratios have poor predictive value for the occurrence of hyperkalemia in ESRD patients.

Our results largely replicate past studies showing poor sensitivity and specificity of peaked T-wave presentation in hyperkalemic patients. ${ }^{6,10,11}$ Aslam et al. found that serum calcium had was a significant variable when using a linear model on T-wave height in ESRD patients. ${ }^{11}$ Our study contradicts this and found that when added as a variable, serum calcium did not affect the correlation of the linear model compared to serum potassium alone. Wrenn et al. found that sensitivity and specificity of the peaked T-wave, as determined by physician readers, mildly improved when predicting severe hyperkalemia $\left(\left[\mathrm{K}^{+}\right]>6.5 \mathrm{mEq} / \mathrm{L}\right)$ compared to hyperkalemia $\left(\left[\mathrm{K}^{+}\right]>5.5 \mathrm{mEq} / \mathrm{L}\right) .{ }^{13}$ We ran two separate ROC analyses for these two serum potassium cutoffs. Our results contradicted Wrenn et al., finding that criterion performance did not significantly change between the two potassium cutoffs.

Our study has several limitations. As this was a retrospective study, several general biases may have affected our conclusions. First, hospital protocol dictated that 12-lead ECGs were taken on those suspected of cardiac ischemia or hyperkalemia, limiting the availability of healthy controls. Additionally, hospital lab protocol did not screen blood samples for falsely elevated potassium levels from hemolysis; however, this was considered insignificant because treating physicians trusted this lab data in clinical decision-making. This study was specific to ESRD patients and does not provide evidence for or against the use of peaked Twaves as a diagnostic criterion for hyperkalemia in patients with normal kidney function. The participants in this study were overwhelmingly Hispanic/Latino, and caution should be taken in applying these results to all races and ethnicities. Additionally, this study examined only the T-wave characteristic on ECG. Future studies should incorporate other variables such as PR interval, QRS duration, and QTc interval, which can be altered by potassium imbalances. ${ }^{7,8}$

\section{CONCLUSIONS}

$\mathrm{T} / \mathrm{R}$ ratios show poor correlation with patients' potassium levels, and peaked T-waves are poor clinical indicators of hyperkalemia in ESRD patients presenting to the emergency department.

\section{ACKNOWLEDGMENTS}

The authors thank Lisa Basgall for her maintenance of the Rice EMS Research Course. The authors also thank Kelly Keene Rogers for her work organizing and maintaining the research project. 


\section{REFERENCES}

1. Einhorn, L. M., Zhan, M., Hsu, V. D., Walker, L. D., Moen, M. F., Seliger, S. L., Weir, M. R., Fink, J. C. The frequency of hyperkalemia and its significance in chronic kidney disease. Arch Intern Med. 2009;169(12):1156-1162. doi:10.1001/archinternmed.2009.132.

2. Levey, A. S., Coresh, J. Chronic kidney disease. Lancet. 2012;379(9811):165-180. doi:10.1016/S0140-6736(11)60178-5.

3. Dillon, J. J., DeSimone, C. V., Sapir, Y., Somers, V. K., Dugan, J. L., Bruce, C. J., Ackerman, M. J., Asirvatham, S. J., Striemer, B. L., Bukartyk, J., Scott, C. G., Bennet, K. E., Mikell, S. B., Ladewig, D. J., Gilles, E. J., Geva, A., Sadot, D., Friedman, P. A. Noninvasive potassium determination using a mathematically processed ECG: Proof of concept for a novel "blood-less, blood test." J Electrocardiol. 2015;48(1):12-18. doi:10.1016/j.jelectrocard.2014.10.002.

4. Hollander-Rodriguez, J. C., Calvert, J. F. Hyperkalemia. Am Fam Physician. 2006;73(2):283-290.

5. Jain, N., Kotla, S., Little, B. B., Weideman, R. A., Brilakis, E. S., Reilly, R. F, Banerjee, S. Predictors of hyperkalemia and death in patients with cardiac and renal disease. Am J Cardiol. 2012;109(10):1510-1513. doi:10.1016/j.amjcard.2012.01.367.

6. Montague, B. T., Ouellette, J. R., Buller, G. K. Retrospective review of the frequency of ECG changes in hyperkalemia. Clin J Am Soc Nephrol. 2008;3(2):324-330. doi:10.2215/CJN.04611007.

7. Parham, W. A., Mehdirad, A. A., Biermann, K. M., Fredman, C. S. Hyperkalemia revisited. Tex Heart Inst J. 2006;33(1):4047.

8. Levis, J. T. ECG Diagnosis: Hyperkalemia. Perm Journal. 2013;17(1):69.

9. Martinez-Vea, A., Bardají, A., Garcia, C., Oliver, J. A. Severe hyperkalemia with minimal electrocardiographic manifestations: a report of seven cases. J Electrocardiol. 1999;32(1):45-49. doi:10.1016/S0022-0736(99)90020-1.

10. Acker, C., Johnson, J., Palevsky, P., Greenberg, A. Hyperkalemia in hospitalized patients: causes, adequacy of treatment, and results of an attempt to improve physician compliance with published therapy guidelines. Arch Intern Med. 1998;158:917924. doi:10.1023/A:1007135517950.

11. Aslam, S., Friedman, E. A., Ifudu, O. Electrocardiography is unreliable in detecting potentially lethal hyperkalaemia in haemodialysis patients. Nephrol Dial Transplant. 2002;17(9):1639-1642. doi:10.1093/ndt/17.9.1639.

12. Sing, T., Sander, O., Beerenwinkel, N., Lengauer, T. ROCR: Visualizing classifier performance in R. Bioinformatics. 2005;21(20):3940-3941. doi:10.1093/bioinformatics/bti623.

13. Wrenn, K. D., Slovis, B. S., Slovis, C. M. The ability of physicians to predict electrolyte deficiency from the ECG. Ann Emerg Med. 1990;19(5):580-583. doi:10.1097/00132586-199212000-00002.

\section{STUDENT AUTHOR}

Ryan Pappal is a fourth-year undergraduate student at Rice University in Houston, TX. A pre-medical student and certified paramedic, Ryan is pursuing degrees in Biochemistry \& Cell Biology and Psychology in preparation for medical school. He will graduate Rice in May 2017.

\section{PRESS RELEASE}

High potassium levels, called hyperkalemia, can cause serious illness in patients. Because kidney disease patients develop hyperkalemia from their disease, we investigated a possible diagnostic criterion from electrocardiograms, called the peaked $\mathrm{T}$ wave, which has long been associated with hyperkalemia. Our results found that the presence or absence of peaked T-waves on electrocardiograms alone are not sufficient to predict whether these kidney disease patients have hyperkalemia or not. Other diagnostic criteria should be developed for the better diagnosis of these patients. 


\title{
Modeling Internet Traffic Generations Based on Individual Users and Activities for Telecommunication Applications
}

\author{
Sara Stoudt ${ }^{* a}$, Pamela Badian-Pessot ${ }^{a}$, Blanche Ngo Mahop ${ }^{a}$, Erika Earley ${ }^{a}$, Jordan Menter ${ }^{a}$, Yadira Flores ${ }^{a}$, Danielle \\ Williams $^{a}$, Weijia Zhang ${ }^{a}$, Liza Maharjan $^{* a}$, Yixin Bao ${ }^{a}$, Laura Rosenbauer $^{a}$, Van Nguyen $^{a}$, Dr. Veena Mendiratta $^{b}$, and \\ Dr. Nessy Tania*a \\ ${ }^{a}$ Department of Mathematics and Statistics, Smith College, Northampton, MA \\ ${ }^{b}$ Bell Labs, NOKIA, Naperville, IL \\ Students: “sstoudt@berkeley.edu,plb93@cornell.edu,bmabop@wpi.edu,erikatearley@gmail.com,jordanlmenter@gmail.com, \\ yadiflores616@gmail.com,dpwilliams1228@gmail.com,wzhang23@smith.edu, *lmaharjan@smith.edu, ybao@smith.edu, \\ lrosenbaner@smith.edu,vnguyen@smith.edu \\ Mentors:"ntania@smith.edu,veena.mendiratta@nokia.com
}

\begin{abstract}
A traffic generation model is a stochastic model of the data flow in a communication network. These models are useful during the development of telecommunication technologies and for analyzing the performance and capacity of various protocols, algorithms, and network topologies. We present here two modeling approaches for simulating internet traffic. In our models, we simulate the length and interarrival times of individual packets, the discrete unit of data transfer over the internet. Our first modeling approach is based on fitting data to known theoretical distributions. The second method utilizes empirical copulae and is completely data driven. Our models were based on internet traffic data generated by different individuals performing specific tasks (e.g. web-browsing, video streaming, and online gaming). When combined, these models can be used to simulate internet traffic from multiple individuals performing typical tasks.
\end{abstract}

\section{KEYWORDS}

Internet Traffic Simulation; Stochastic Models; Empirical Copula; Cumulative distribution function; Wireshark

\section{INTRODUCTION}

Internet traffic modeling and simulation are useful tools for testing new telecommunication technologies and analyzing the performance and capacity of various network protocols, topologies, and algorithms. The goal of our work was to build mathematical models of internet traffic for multiple users using different applications within the same computer network. Specifically, we collected and analyzed internet traffic data generated by individual users performing specific tasks (e.g. web browsing, e-mailing, video streaming, and online gaming) and then used this data to build mathematical models that simulate internet traffic. Our model takes as inputs the number of users and the proportion of internet applications being used at a given time and outputs artificial traffic data.

Data is transferred over the internet in discrete units known as packets rather than in continuous streams. Each packet can vary in size/length (measured in units of bytes) up to a maximum size that may be imposed by the local network that a user is connected to. Thus, internet traffic consists of the transfers of individual packets, where each packet can be characterized by two random variables: interarrival time and size. We began data collection by capturing the internet traffic generated by a single user utilizing one application. From the data, we built models each describing packet transfers for one user on a single application. We then combined these models to imitate the traffic generated by multiple users using different applications. 
As the usage and structure of the internet rapidly changes, mathematical and statistical models for internet traffic are in constant demand. Cleveland et. al. ${ }^{1}$ provide the fundamentals of the information found in internet traffic data, and Sanchez et. al. ${ }^{7}$ discuss first steps for understanding patterns and properties of interarrival times of packets and bytes per packet. We consider two main approaches to modeling internet traffic data: fitting theoretical distributions to interarrival times and packet size, and building empirical models from representative data. Luo et. al. ${ }^{3}$ provide an internet traffic simulation case study whose main features include the fitting of theoretical distributions and visual validation of simulated data. Mah ${ }^{4}$ provides an example of an empirical model to describe internet usage. Dainotti et. al. ${ }^{2}$ mention the lack of literature about modeling packets and bytes jointly. Possolo ${ }^{5}$ describes the background for a copula which can empirically model two distributions jointly. In this paper, we explore both modeling frameworks: theoretical model fitting (by working with known distributions such as the exponential and normal distributions) and empirical model building. We compare and contrast the two methods and discuss the strength and weaknesses of the two approaches.

Additionally, we also consider two different frameworks for modeling internet traffic. We can model at the individual packet level, simulating interarrival time and bytes per packet; alternatively, we can also aggregate and predict the number of packets and total bytes that are transferred per second. The application for the model determines which framework is more appropriate. If high granularity is a focus, flow of individual packets should be used. However, the interarrival times between packets can be extremely short (order of nanoseconds). Therefore, if a simulation over a longer time frame is needed, modeling packets per second is often more computationally feasible. We constructed our theoretical model using the first framework; for our empirical model, we use the second approach.

\section{METHODS AND PROCEDURES}

Data Collection and Preliminary Analysis

Prior to building our computational models, we first collected internet traffic data using Wireshark ${ }^{8}$, an open-source packet analyzer. Here, individuals connected to an internet network from their personal computers and captured their packet data using Wireshark for a specific time period (e.g. 10, 15, or 60 minutes). Their internet activity was limited to one of the following: web browsing, video streaming, or online gaming. Most of the data sets were obtained from users connecting to the local wireless network at Smith College. For our mathematical models, we used data collected for 60 minute intervals and focused on two quantities: the packet size and interarrival time of packets. The interarrival times, which are the time intervals between consecutive packets, are obtained by first sorting the timestamps of packet arrivals and then subtracting the consecutive times.

\section{Theoretical Models}

Our first modeling approach was to fit the histograms of interarrival time and packet size to a number of commonly used theoretical distributions. Here, we treated the packet size and the interarrival time as two independent random variables. Further on, we describe an empirical modeling approach where the two quantities are described by a joint bivariate probability distribution with no independence assumptions.

One common model for describing waiting times is the exponential distribution based upon the assumption that there is a specific constant probability of an event occurring within a short period of time (constant probability rate) ${ }^{6}$. We found that packet interarrival times cannot be described by a single exponential distribution. Rather, they are better described by a hyperexponential distribution which describes a mixture of several exponential distributions (different rates, each chosen with specific probability). More concretely, we assume that packets can be grouped into multiple categories (e.g. by protocol used, or by whether it is a packet header, trailer, or a payload), labeled as $i=1,2,3, \ldots, N$. A packet of category $i$ is generated with probability $p_{i}$; within category $i$, the interarrival time of packets follows an exponential distribution with rate $\lambda_{i}$. Then, the probability density function (PDF) and the cumulative distribution function (CDF) for the interarrival 
times of packets (random variable $t$ ) can be written respectively as:

$$
\text { PDF: } f(t)=\sum_{i=1}^{N} p_{i} \cdot \lambda_{i} \exp \left(-\lambda_{i} t\right), \quad \text { CDF: } F(t)=1-\sum_{i=1}^{N} p_{i} \cdot \exp \left(-\lambda_{i} t\right) .
$$

Equation 1.

To minimize the number of unknown parameters, we chose $N=3$ (this was based on trial and error, we found that $\mathrm{N}=3$ is the minimal number needed to obtain a good fit to the data). We obtained parameter values for $\lambda_{i}$ and $p_{i}$ by grouping the data into three intervals: $\left[0, T_{1}\right),\left[T_{1}, T_{2}\right]$, and strictly greater than $T_{2}$. Within each group/interval, we set $p_{i}$ as the fraction of the data that lies within the interval and $\lambda_{i}$ as the inverse of the mean of the data within the corresponding interval (maximum likelihood estimate for the rate parameter of an exponential distribution). We obtained the values for $T_{1}$ and $T_{2}$ by performing nonlinear least-squares optimization minimizing the difference between the CDF given in Equation 1. and the empirical CDF (ECDF) from the data. Denote $\left\{\tau_{k}\right\}_{k=1}^{M}$ as the interarrival time data, sorted from shortest to longest, then the ECDF is a step function with values given by $F_{E}\left(\tau_{k}\right)=k / M$ where $k=1,2, \cdots, M$. The square-difference between the two CDFs is then computed according to

$$
D\left(T_{1}, T_{2}\right)=\sum_{k=1}^{M}\left(F_{E}\left(\tau_{k}\right)-F\left(\tau_{k} ; T_{1}, T_{2}\right)\right)^{2} .
$$

Equation 2.

Note that the value of the CDF $F$ in Equation 1. is dependent on the parameter values $\left\{p_{i}, \lambda_{i}\right\}$ which in turn are dependent on the values of the interval end-points $T_{1}$ and $T_{2}$. Minimization of the square-difference function $D$ is done by using the Matlab function fmincon $\left(T_{1}\right.$ and $T_{2}$ are constrained so that $\left.0<T_{1}, T_{2}<\tau_{M}\right)$.

To simulate an interarrival time for the next packet (random variable $t$ ), we draw from the probability distribution in Equation 1. as follows:

1. Generate a pair of random variables $r_{1}$ and $r_{2}$ from the uniform distribution in $[0,1]$.

2. If $r_{1} \leq p_{1}$ then choose the rate $\lambda_{1}$ and convert $r_{2}$ into an exponentially distributed random variable via $t=$ $-\log \left(r_{2}\right) / \lambda_{1}$.

3. Else if $p_{1}<r_{1} \leq p_{2}$, then choose rate $\lambda_{2}$ and $t=-\log \left(r_{2}\right) / \lambda_{2}$.

4. Otherwise, $r_{1}>p_{2}$, so choose rate $\lambda_{3}$ and $t=-\log \left(r_{2}\right) / \lambda_{3}$.

As will be discussed later on (see histogram on Figure 4), packet length distributions generated by different applications tend to be bimodal, so we fitted the data to two different normal distributions separated by a uniform distribution. Thus, we again consider three different possibilities: a packet length is generated with probability $f_{1}$ from a normal distribution with mean $\mu_{1}$ and standard deviation $\sigma_{1}$, with probability $f_{2}$ from a normal distribution with mean $\mu_{2}$ and standard deviation $\sigma_{2}$, and with probability $f_{3}=1-\left(f_{1}+f_{2}\right)$ from a uniform distribution in $\left[L_{1}, L_{2}\right]$. Then the cumulative distribution function for the packet size (random variable $l$ ) can be written by combining the CDFs for the normal and uniform distributions:

$$
G(l)=\frac{f_{1}}{2}\left[1+\operatorname{erf}\left(\frac{l-\mu_{1}}{\sqrt{2 \sigma_{1}^{2}}}\right)\right]+\frac{f_{2}}{2}\left[1+\operatorname{erf}\left(\frac{l-\mu_{2}}{\sqrt{2 \sigma_{2}^{2}}}\right)\right]+f_{3}\left[\frac{l-L_{1}}{L_{2}-L 1}\right] .
$$

Equation 3.

For data-fitting, we again binned the data by choosing two cut-off values, $L_{1}$ and $L_{2}$. Further inspection of the data revealed that a proportion of the packet length data has a constant value of 1440 bytes (likely the standard size for payload packets in our network), so we use $L_{2}=1440$ bytes and set $\mu_{2}=1440$ and $\sigma_{2}=0$ (mean and standard deviation of the normal distribution corresponding to the second peak in the bimodal histogram). $L_{1}$ is chosen through a least-squares procedure. 
The algorithm for drawing from this packet length distribution is also similar to that for interarrival time. However, rather than generating a random variable that is exponentially distributed, here we use either a normal or uniform distribution.

The gaming application is a special case as the packet length distribution is not bimodal and has an exponential shape instead. We observed that online gaming can be more data intensive (e.g. some packets are larger than 4000 bytes) and the packet size has a higher standard deviation than other applications. To describe the packet size distribution for gaming, we fit the data to an exponential distribution with rate $\alpha$. The parameter values obtained from fitting the data for different applications are listed in Table 1.

\section{Interarrival Times}

\section{Video Streaming}

Online

Gaming

\begin{tabular}{|l|l|}
\hline Rates $(/ \mathrm{s})$ & Frequency \\
\hline$\lambda_{1}=2.68 \mathrm{e}+05$ & $p_{1}=0.2706$ \\
\hline$\lambda_{2}=1.42 \mathrm{e}+03$ & $p_{2}=0.6997$ \\
\hline$\lambda_{3}=4.58$ & $p_{3}=0.0297$ \\
\hline
\end{tabular}

\begin{tabular}{|l|l|}
\hline Rates $(/ \mathrm{s})$ & Frequency \\
\hline$\lambda_{1}=3.97 \mathrm{e}+05$ & $p_{1}=0.3851$ \\
\hline$\lambda_{2}=1.26 \mathrm{e}+03$ & $p_{2}=0.3813$ \\
\hline$\lambda_{3}=7.63$ & $p_{3}=0.2285$ \\
\hline
\end{tabular}

\begin{tabular}{|l|l|}
\hline Rates $(/ \mathrm{s})$ & Frequency \\
\hline$\lambda_{1}=1.14 \mathrm{e}+04$ & $p_{1}=0.3604$ \\
\hline$\lambda_{2}=59.03$ & $p_{2}=0.6371$ \\
\hline$\lambda_{3}=5.88$ & $p_{3}=0.0025$ \\
\hline
\end{tabular}

Packet Length

\begin{tabular}{|ll|l|}
\hline Parameters & Frequency \\
\hline$\mu_{1}=60.24, \quad \sigma_{1}=10.80$ & $f_{1}=0.3694$ \\
\hline$\mu_{2}=1440, \quad \sigma_{2}=0$ & $f_{2}=0.5930$ \\
\hline \multicolumn{2}{|c|}{ Uniform $[100,1440]$} & $f_{3}=0.0376$ \\
\hline
\end{tabular}

\begin{tabular}{|cc|c|}
\hline \multicolumn{2}{|l|}{ Parameters } & Frequency \\
\hline$\mu_{1}=69.09$ & $\sigma_{1}=18.15$ & $f_{1}=0.5640$ \\
\hline$\mu_{2}=1440$ & $\sigma_{2}=0$ & $f_{2}=0.2824$ \\
\hline \multicolumn{2}{|c|}{ Uniform $[120,1440]$} & $f_{3}=0.1536$ \\
\hline
\end{tabular}

\begin{tabular}{|l|l|}
\hline Parameters & Frequency \\
\hline$\alpha=0.0068$ & 1 \\
\hline
\end{tabular}

Table 1. Table of parameter values for different applications.

\section{Empirical Copula Method}

As an alternative to theoretical distributions, we use the copula method to simulate internet traffic. The copula is a flexible, non-parametric approach that does not rely on any distributional assumptions. On the other hand, we may not obtain certain information that convey average behaviors that can be inferred from parameter values obtained from data-fitting to the previous theoretical models.

The copula method can be used for internet traffic simulation at a fine individual packet level (interarrival times in the order of nano seconds). However, this approach is not computationally feasible for simulations with longer time scales, so we instead simulated the number of packets and total bytes that are generated per second. Either way, the copula method allows us to model the two random variables of interest jointly, rather than assuming that they are independent. This method is completely data driven which allows it to be easily applied to any data set. Different copulae can be made to reflect different types of users based on usage, time of day, spatial location, etc. We model user variability by modeling each type of application separately. However, we did not distinguish between different users performing the same task.

Based on a data set from one user with a specific task, we can build a copula as follows:

1. Find the empirical cumulative distributions for packets-per-second and bytes-per-second.

2. Map the corresponding ECDF values to a unit square by using the ECDF of packets-per-second as the $x$-coordinate and the total bytes-per-second as the $y$-coordinate.

These steps are schematically shown in Figure 1.

Once we generate a copula surface, we can draw from this joint distribution to obtain the two random variables of interests as follows (see schematics illustration in Figure 2): 
1. Simulate packets-per-second by pulling a random number $x$ from the uniform distribution in $[0,1]$. This gives an ECDF value for the packets-per-second variable (shown in Figure 1) which can then be inverted to obtain the packetsper-second value.

2. Next, simulate bytes-per-second conditional on $x$; this is akin to taking a slice of the copula surface. Fixing $x$, draw from the probability distribution function for the ECDF values for the second variable as illustrated in Figure 2. This random number (in $[0,1])$ corresponds to an ECDF value and yields the simulated value for bytes-per-second after inversion.

3. Repeat this process many times until the elapsed time is achieved. Each pair of random variables from steps 1-2 above is a simulated second of internet traffic for one user. We can combine simulations from different copulae in a simulator.

\section{Step 1: Generate ECDF}
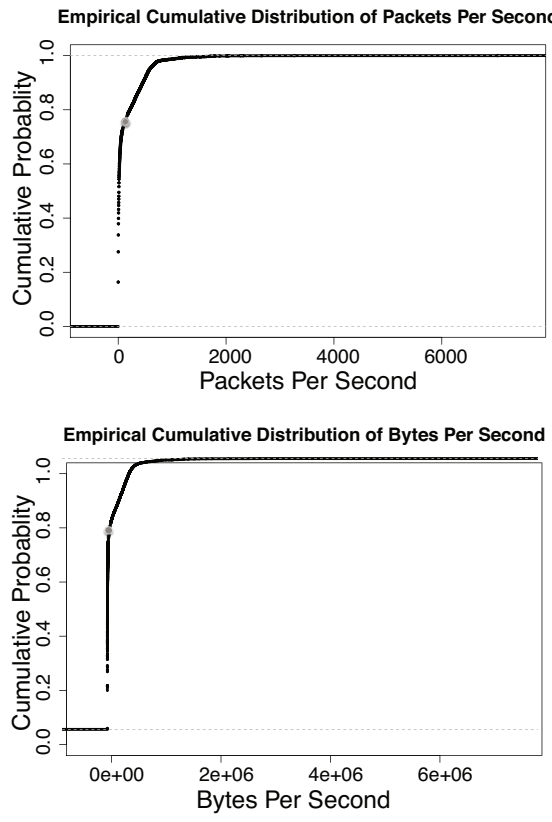

\section{Step 2: Map to Unit Square}

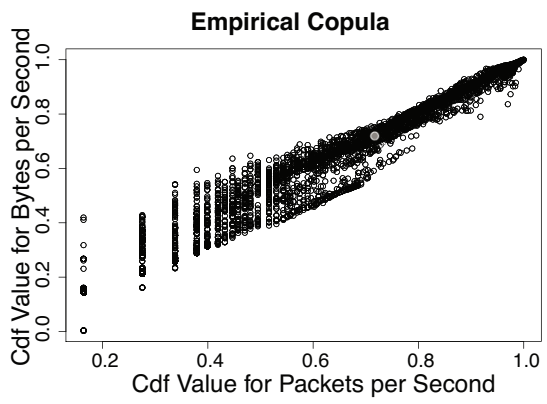

Figure 1. Schematics of steps for building copula.
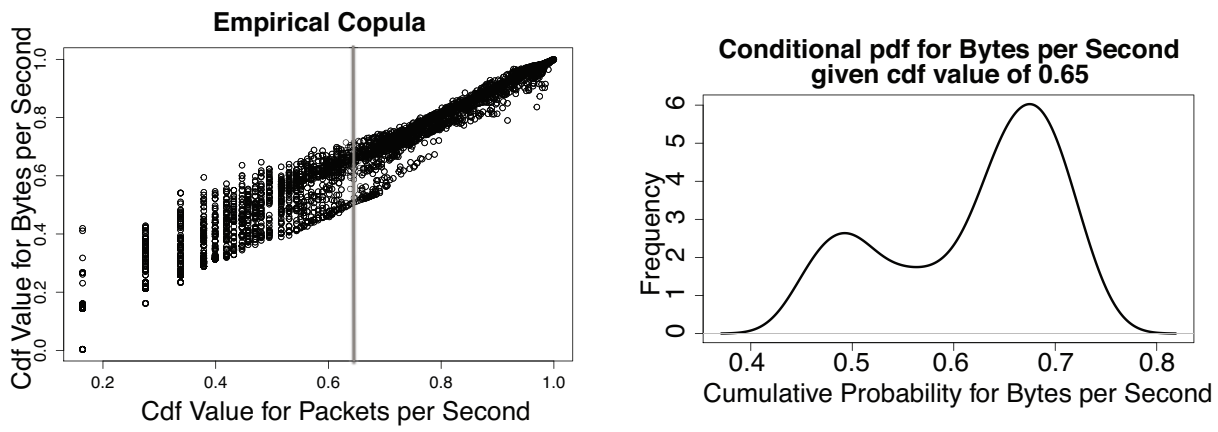

Figure 2. Schematics for simulating from a copula. 


\section{RESULTS AND DISCUSSION}

\section{Preliminary Data Analysis}

Prior to building and simulating the computational models, we analyzed the interarrival time and packet length data collected from a single user performing specific applications for 60 minutes. In Figure 3, we show the density plots (normalized histogram) for the distribution of interarrival times for different applications. Here, the interarrival time is shown in log scale as their values are typically short, but can vary by orders of magnitudes. The density plots showing the distribution of packet sizes are shown in Figure 4. With the exception of online gaming, the distribution of packet size appears to be bimodal (distributions with two peaks). We expect that the smaller packets carry the "control information" (packet headers/trailers) that contain short information needed to deliver the packet, while the larger packets contain the actual data ("payload").
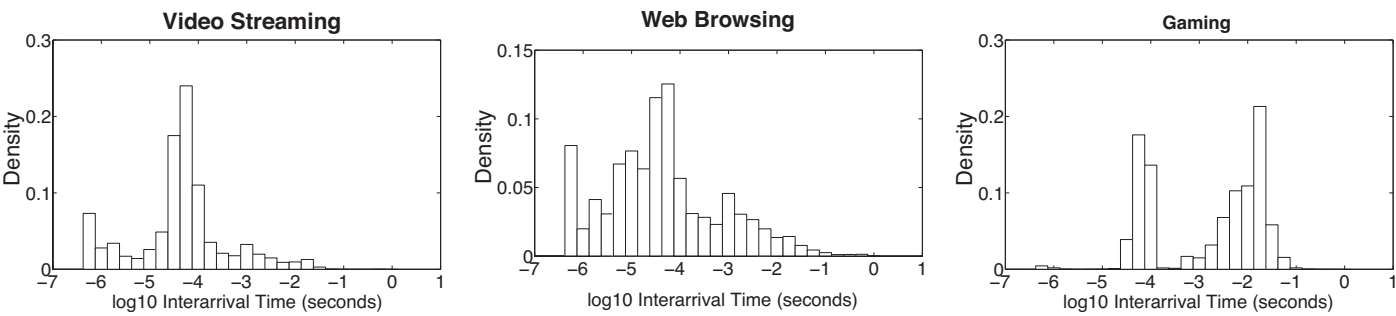

Figure 3. Density plots of interarrival time for applications. Each histogram corresponds to one data set collected from one user performing one task for 60 minutes.
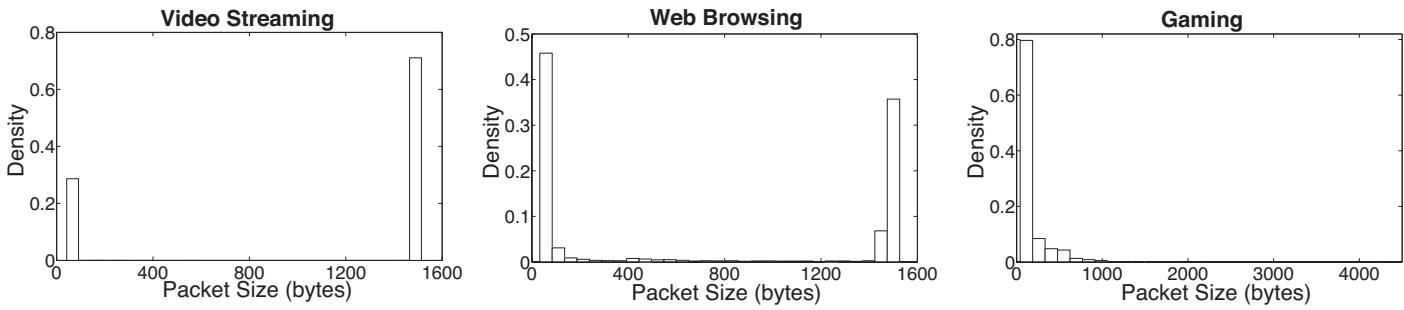

Figure 4. Density plots of packet sizes for different applications. Each histogram corresponds to one data set collected from one user performing one task for 60 minutes.

\section{Simulation Results from Theoretical Models}

Using parameter values obtained from data fitting in Table 1, we first assessed the fit of the model (using parameter values obtained from least-square optimization) to the data. In Figure 5, we compared the cumulative distribution functions from the model to the empirical cumulative distribution functions of the data. The models fit the data relatively closely though the models do not fully capture the variations in the data. We then simulated the timing and size of individual packets by drawing from respective theoretical distributions given in Equation 1., Equation 3. Combining the theoretical distributions for different applications (parameter values listed in Table 1), traffic for multiple users can be simulated. For simplicity, we assume that each user only performs a single task but we can specify the proportions of users performing each activities. On Figure 6, we show the traffic generated by 15 users (10\% gaming, 40\% video streaming, and 50\% web browsing).

One advantage of using the theoretical method is fast computation at a fine packet size level. Here, the random variables of interest (namely packet lengths and interarrival times) can be generated quickly as they involve standard distributions (normal, uniform, or exponential) that can be inverted/transformed easily.

On the other hand, data-fitting to theoretical distributions also presents some limitations. First, we assumed that the interarrival times and packet lengths are two independent random variables. In reality, these may be dependent; for example, 

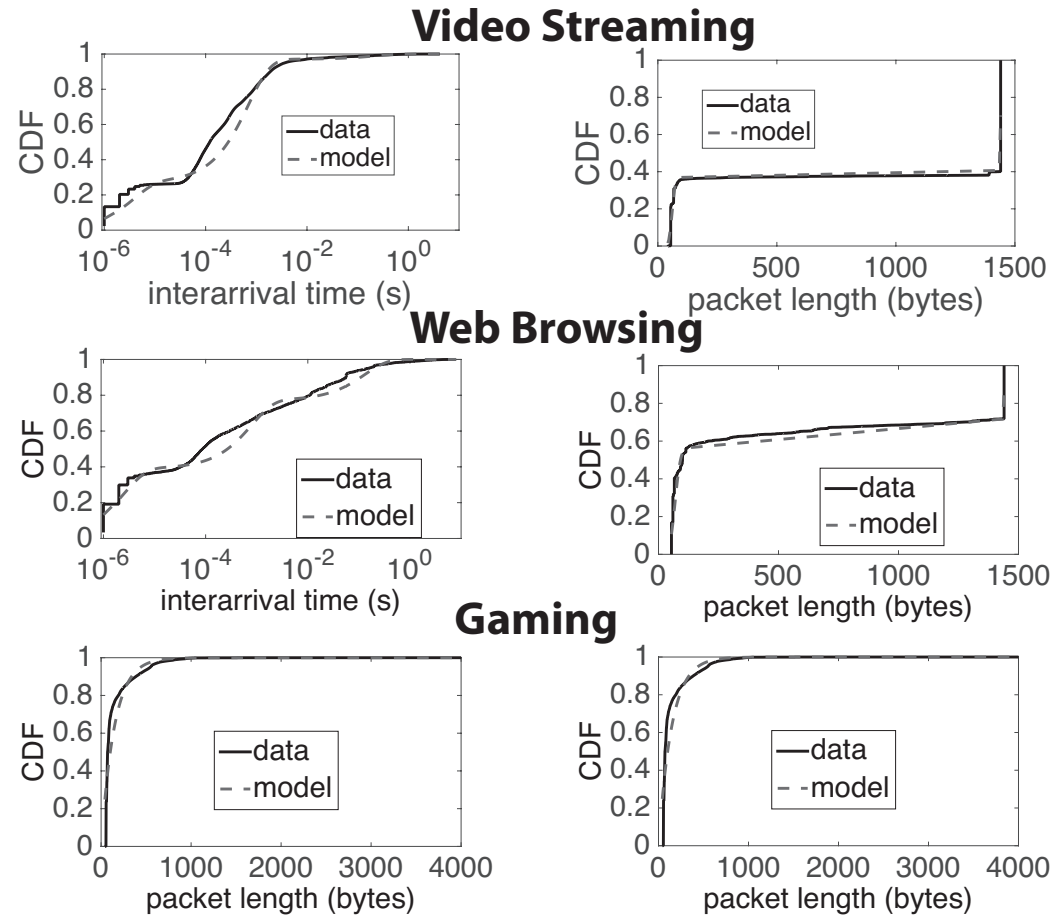

Figure 5. Data fitting results: comparison of ECDFs from data and simulation from theoretical models for different activities.

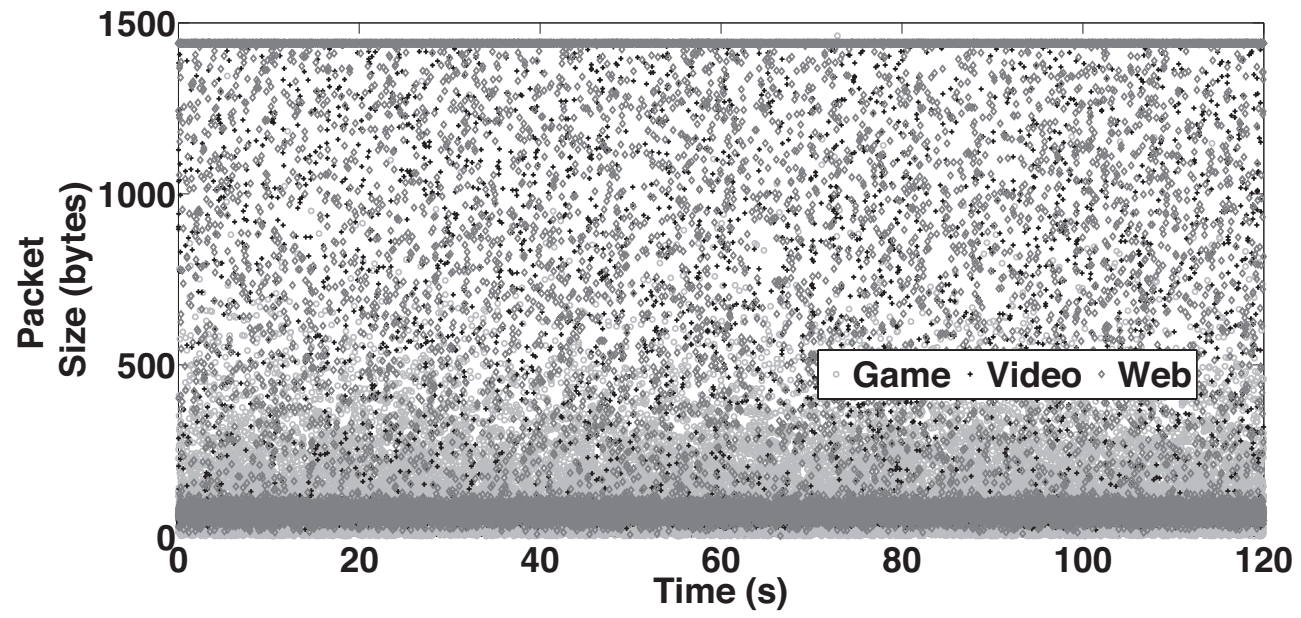

Figure 6. Internet traffic simulation obtained from the theoretical model for 15 users performing the following fractions of activities: (10\% gaming, $40 \%$ video streaming, and $50 \%$ web browsing).

smaller packets carrying control information may be generated more quickly to establish communications between computers, while larger packets carrying payloads are dependent on user activities (longer time scale than nanoseconds) and may be limited by the speed of data transfer (bandwidth cap) imposed by the network. Next, the simulation results are also dependent on the parameter values, which can vary significantly from one data set to the next. The least squares data-fitting procedures can be sensitive to the choice of initial guesses. We now turn to an empirical method that allows us to overcome some of these limitations. 


\section{Simulation Results from Empirical Copula Method}

We first built the copula surface for each activity and plotted the copulae in Figure 7. Each activity generates a copula with distinct features, confirming our approach that we should model them separately. Combining the different copulae, we can simulate multiple users performing different tasks. A simulation of internet traffic for 10 users (25\% Video, 50\% Web, 25\% Gaming) over the course of an hour is shown in Figure 8. Computational costs associated with the copula method are higher as the method requires repeated resampling of a large data set. Here we reduced the computational time by considering the number of packets and bytes transferred in a given second, rather than simulating individual packets.
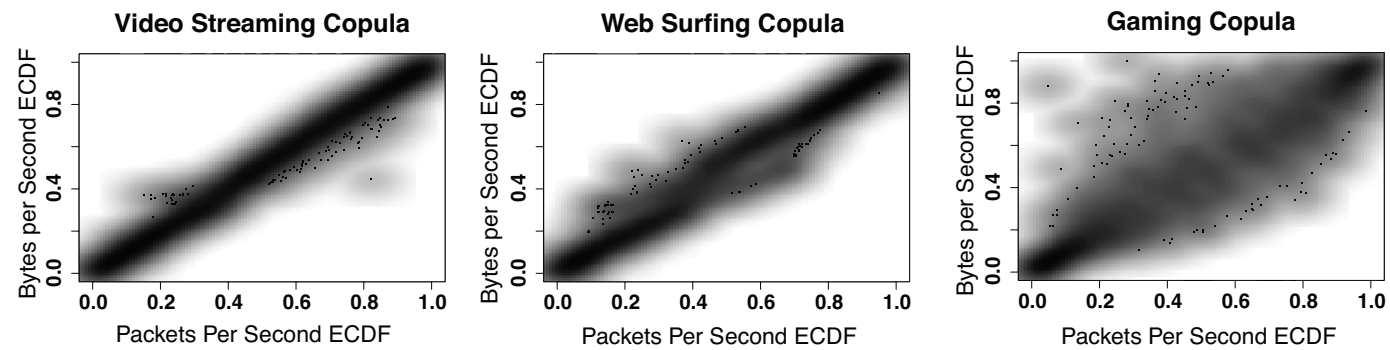

Figure 7. Copulae for different activities.

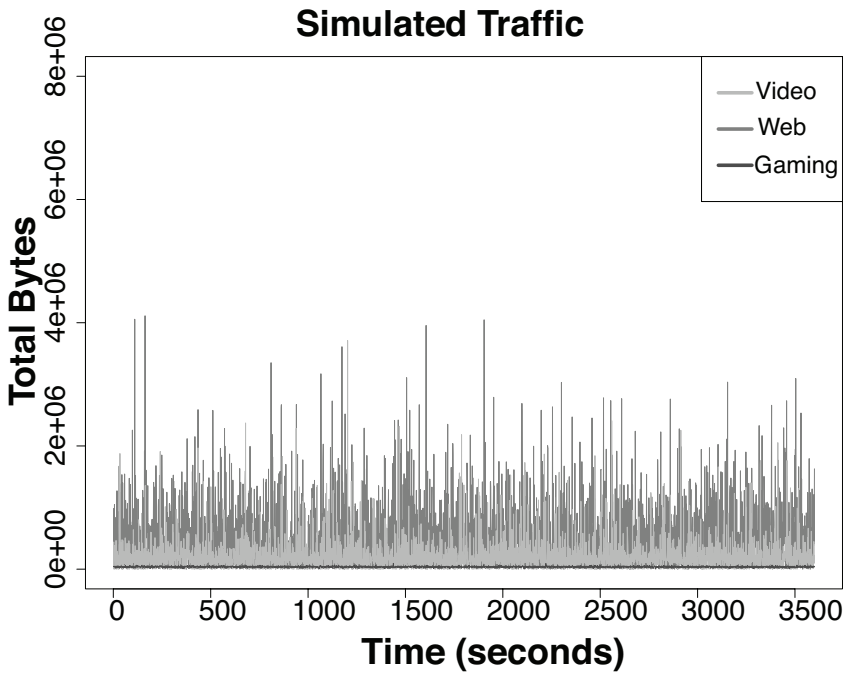

Figure 8. A simulation from the copula method for internet traffic for 1 hour from 10 users (25\% video, 50\%, and $25 \%$ gaming).

\section{Model Verifications and Comparisons}

We first compared simulations from both the theoretical model and the empirical model. To do this, we built each model on the same data sets and generated simulations based on the same inputs (number of users, time frame, and proportion of users performing each activity). Since the copula method is completely data driven and a finer granularity gives us more data to build the copula, we wanted to make sure that the empirical method was not adversely affected by our choice of fairly small data sets. Therefore, we compared results at the second level, half-second level, and quarter second level to ensure that our time scale does not affect the comparisons. For brevity, we show the results at the second level here, but the results from the half-second and quarter second levels were similar.

First, we assess the simulations from these two methods visually. We can see in Figure 9 that the simulations from the theoretical model have less variability than those from the empirical method. It is reassuring to see a sense of consistency; 
both models have a similar center. Depending on the application, there could be advantages to having different properties in the simulations. For capacity testing, it may be beneficial to have simulations that have higher variability and capture the information in the tails of the internet traffic. For an application where we are more interested in ascertaining information about the "average" user, the theoretical model may be preferred.

15 Users: Packets Per Second

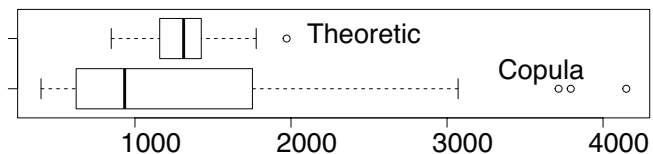

15 Users: KBytes Per Second

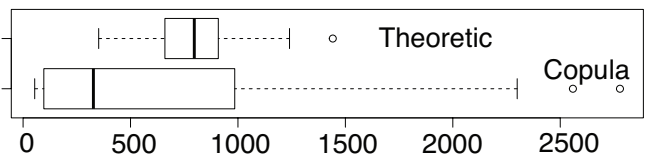

Figure 9. Comparison of simulation results from theoretical and empirical (copula) models.

We then measure the goodness of fit of both models. However, the empirical copula method is non-parametric (no parameter fitting was performed). Therefore, standard goodness of fit techniques for assessing how well the model conforms to the data, do not apply. Therefore we need another way to assess fit. One way we can do this is to mimic the KolmogorovSmirnov test; this test measures the maximum difference between the values of the empirical cumulative distributions from model simulations and the data.

To get a sense of how this would change given different data, we can "shuffle" our data set (by drawing with replacement a sample of 1000 points from the full data set) and then compute the maximum difference in empirical cumulative distribution functions. When we do this many times, we get a distribution for our maximum difference. We can do this for many different types of methods, including the theoretical model, to get a sense for how well each model works. To put our results in context we can test a naive model as our "worst case scenario" and a true data shuffle as our "best case scenario." We choose our naive model to be a uniform copula, a flat unit square.

In Figure 10, we can see that, as expected, the simple resampling of the true data yields the smallest maximum difference centered, for example around 0.05 for web packets, and the uniform copula yields the largest maximum difference centered around 0.912 for web packets. The copula method yields maximum difference values closer to those of the resampling. In fact, the distributions for these two overlap. The theoretical method has larger maximum differences but note that here we were comparing coarser grained result (per-second level) while data-fitting for the theoretical model was done at the fine individual-packet level. The same relative ordering of the goodness of fit measure holds for the packets and bytes for web surfing, video streaming, and online gaming with the distributions for resampling and the copula overlapping, and the distributions for the theoretical method being closest to those of the uniform copula method. Nonetheless, the theoretical model still yields maximum differences that are smaller than the uniform copula method.

\section{CONCLUSIONS}

We have described two approaches for generating artificial internet traffic consisting of packet lengths and interarrival times simulations. After collecting individual user data, we produced models that describe traffic generated during web surfing, video streaming, and gaming, which were then combined to form an internet traffic simulator. We considered two methods. The first method is based on fitting known theoretical distributions to the data to simulate individual packets; the second method used an empirical copula to simulate packets per second. The theoretical model allows for fast computation, but packet length and interarrival time are assumed to be independent variables. This limitation is removed when the copula method is used. However, the copula method can be computationally expensive, especially for simulating individual packets with very short interarrival times. To speed up computations, we simulated packets per second instead. When we evaluated how well each methods capture the underlying data, we found that the theoretical method models the center of the distribution well but does not capture the tails as well as the empirical method. Both models show significant improvement over a naive approach with the empirical method slightly outperforming the theoretical method (measured by KolmogorovSmirnov statistics i.e. ECDF max difference for comparison). 
Web Packets- Resample

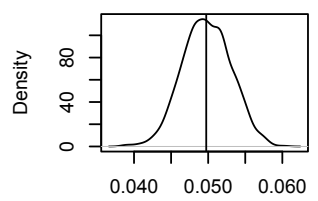

max difference

Web Bytes- Resample

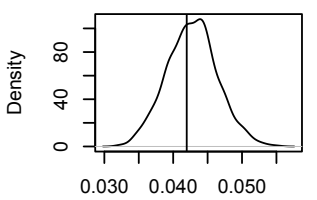

max difference

Video Packets- Resample

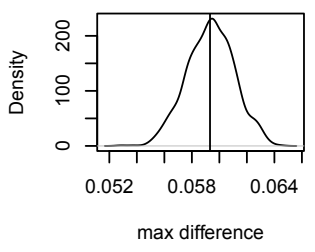

Video Bytes- Resample

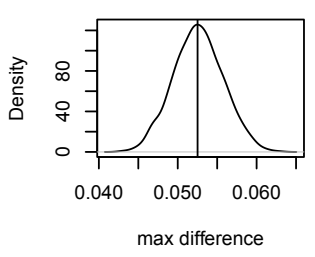

Game Packets- Resample

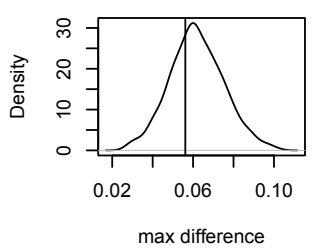

Game Bytes- Resample

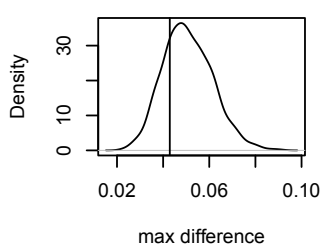

Web Packets- Copula

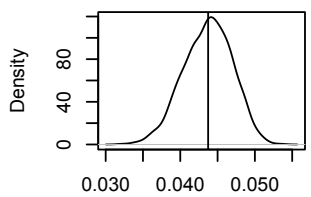

max difference

Web Bytes- Copula

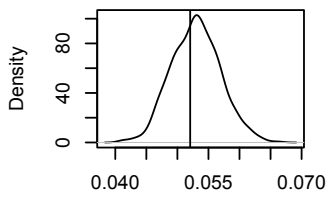

max difference

Video Packets- Copula

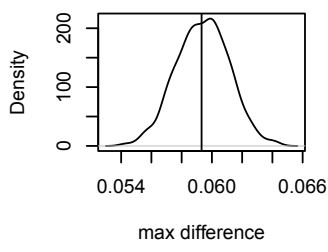

Video Bytes- Copula

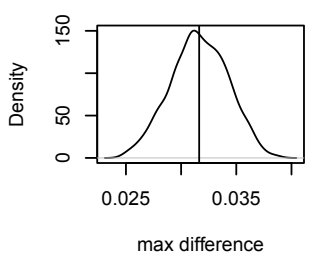

Game Packets- Copula

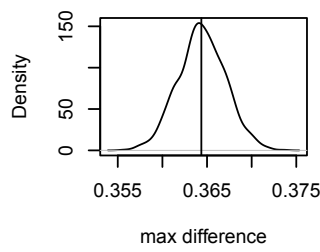

Game Bytes- Copula

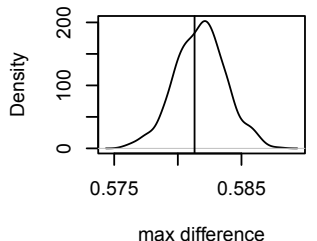

Web Packets- Theoretical

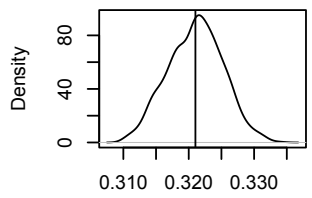

max difference

Web Bytes- Theoretical

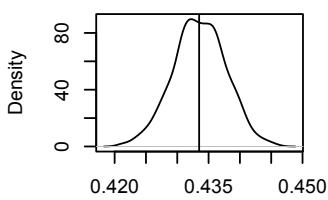

max difference

Video Packets- Theoretica
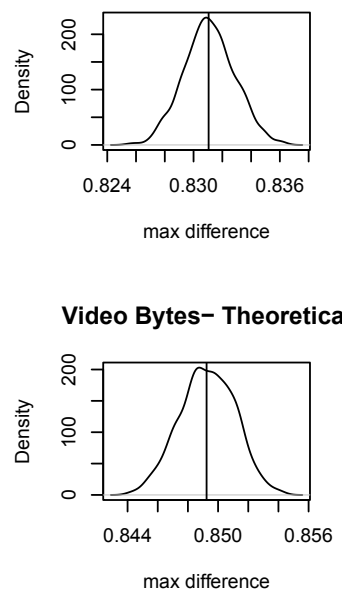

Game Packets- Theoretica

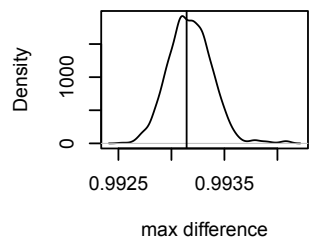

Game Bytes- Theoretical

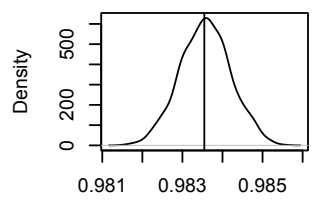

max difference
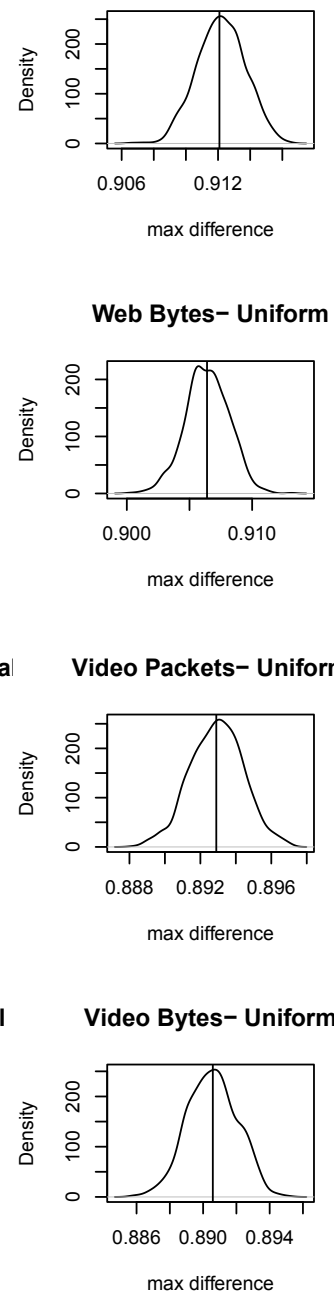

max difference

Web Bytes- Uniform

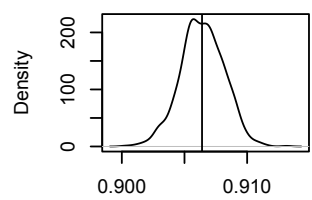

max difference

Video Packets- Uniform

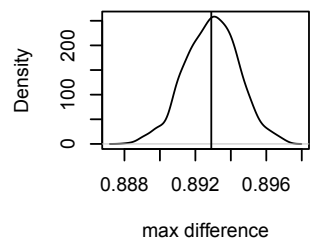

ideo Bytes- Uniform

Game Packets- Uniform

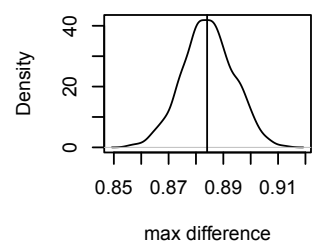

Game Bytes- Uniform

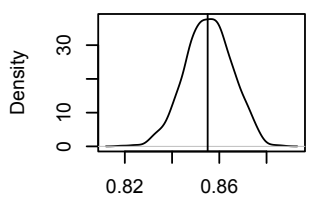

max difference

Figure 10. Maximum difference in ECDF comparisons. 
The methods presented here form an underlying basis for developing more realistic models in the future. Here, we do not include any network signaling effects, which describe the relationship between user requests and network response. There is a difference between what a user asks for and what the network returns; this may depend on the network structure itself as well as the load or number of users utilizing the network. For example, if many users are inundating the network with requests, it may return less than the requested amount per user based on a capacity constraint. Our traffic simulation results can also be compared to a larger data set from a network utilized by multiple users at different time periods during the day (capturing varying traffic loads and the network limitation). We can then also include suitable dampening effects in our simulator as the number of users increases.

Each distinct protocol used for packet transfer may exhibit different interarrival time and packet length distributions as each protocol serves different functions (e.g. security/encryption, peer-to-peer network establishment, or information transfer). In the future, more realistic models which take account protocol signaling can be developed. To further explore these effects, the Wireshark data can first be reanalyzed and the model can be expanded to include the directions of packet transfers. Protocol signaling may also lead to bursts of packet transfers. The theoretical method presented here is based on Poisson processes with no memory thus does not capture any bursting behavior. Packet simulations using the copula method are also memory-less in that the generation of consecutive packets are independent from one another; however, bursting tendencies may be partially captured if there is a higher density for shorter interarrival times in the copula surface. Further analyses of correlations of interarrival times or size between consecutive packets is an interesting future direction in order to investigate the extent of "memory" between consecutive packet transfers.

Another possible route to take in the future is the collection of a wider variety of applications that generate internet traffic, e.g. music streaming, file-sharing, and real time audio sessions (e.g. with Skype and WhatsApp). As we build up these approaches with a wider variety of applications, it will be interesting to compare the performance of our models with standardized internet traffic simulators such as the Third Generation Partnership Project 2 (3GPP2) ${ }^{9}$ and the ns-3 Network Simulator ${ }^{10}$.

\section{ACKNOWLEDGEMENTS}

This project was funded by NSF grant DMS-1345499 through the MAA Preparation for Industrial Careers in Mathematical Sciences Program (PIC Math) and NSF grant DMS-1143716 for the Center for Women in Mathematics, Smith College. We thank Smith College ITS for consultations on campus-wide data. We are also grateful for helpful feedback from other research teams at Bell Labs.

\section{REFERENCES}

1. Cleveland, W.S. and Sun, D.X. (2000) Internet Traffic Data, J Am Stat Assoc 95, 979-985.

2. Dainotti, A., Pescape, A., Rossi, P.S., Palmieri, F., and Ventre, G. (2008) Internet Traffic Modeling by Means of Hidden Markov Models, Comput Netw 52, 2645-2662.

3. Luo, S., and Marin, G.A. (2005) Realistic Internet Traffic Simulation Through Mixture Modeling and a Case Study, in Proceedings of the 37th Conference on Winter Simulation, 2408-2416.

4. Mah, B.A. (1997) An Empirical Model of HTTP Network Traffic, in Proc Infocom '97, 592-600.

5. Possolo, A (2010) Copulas for Uncertainty Analysis, Metrologia 47, 262-271.

6. Ross, S (2002) A First Course in Probability, 6th ed., Prentice Hall, New Jersey.

7. Sanchez, J. and He, Y (2005) Internet Data Analysis for the Undergraduate Statistics Curriculum, J Stat Educ 13, 1-20.

8. Wireshark, development release 1.12.5, https://www.wireshark.org (accessed June 2016).

9. Third Generation Partnership Project 2, http://wwrw.3gpp2.org/(accessed August 2016).

10. ns-3 Network Simulator, https://wwre.nsnam.org/(accessed August 2016). 


\section{ABOUT THE STUDENT AUTHORS}

Sara Stoudt is currently a Ph.D. student in the Department of Statistics at the University of California, Berkeley. She is also a participant in the Environment and Society: Data Science for the 21st Century training program and a National Physical Science Consortium Fellow with the National Institute of Standards and Technology. She completed a B.A. in Mathematics from Smith College in 2015. She is interested in applied and computational statistics with emphasis on environmental data problems.

Pamela Badian-Pessot is currently a Ph.D. student at the School of Operations Research, Cornell University. She completed a B.A. in Mathematics and Economics from Wells College in 2013, and the Post-Baccalaureate Program at the Center for Women in Mathematics, Smith College in 2015.

Blanche Ngo Mahop is currently pursuing a Ph.D. in Computational and Applied Mathematics at the Worcester Polytechnic Institute. She completed a B.S. in Applied Mathematics from Howard University in 2014, and the Post-Baccalaureate Program at the Center for Women in Mathematics, Smith College in 2015.

Erika Earley is currently working as a resident software engineer at Google in New York City. She completed a B.A. in Mathematics from Smith College in 2015.

Jordan Menter is currently working as a Data Analytics Consultant with the MassMutual Data Science Development Program in Amherst, MA. She will begin pursuing an M.S. in Computer Science at the University of Massachusetts, Amherst in the fall of 2016. She completed a B.A. in Mathematics from Smith College in 2016.

Yadira Flores is currently an Application Developer for Wharton Research Data Services in Philadelphia, PA. She completed a B.A. in Mathematics from Smith College in 2015.

Danielle Williams is currently pursuing a career in education in the School District of Philadelphia. She completed a B.S. in Mathematics and Secondary Education from East Stroudsburg University in 2014, and the Post-Baccalaureate Program at the Center for Women in Mathematics, Smith College in 2015.

Weijia Zhang (Vega) is currently interning for the Quantitative Management Associate Program at Bank of America. She is pursuing her B.A. in Mathematics and Statistics at Smith College. She is interested in using quantitative skills solving problems, especially in the field of finance.

Liza Maharjan is a current undergraduate student at Smith College pursuing her undergraduate degree in Mathematics and Economics. She plans to pursue an M.Phil. in Development Studies at Oxford University following graduation in 2017.

Yixin Bao is currently pursuing her B.A. in Mathematics at Smith College. She also studied abroad at the London School of Economics, Department of Statistics during her junior year.

Laura Rosenbauer is currently pursuing her B.S. in Engineering at Smith College.

Van Nguyen is currently pursuing her B.A. in Computer Science and Economics at Smith College. 


\section{PRESS SUMMARY}

Computational models that can generate artificial internet traffic are useful tools for testing the limits and robustness of new telecommunication technologies. In this paper, we collected data in order to build computational models that take into account the arrival time of packets (units for information transfer over the internet) and their sizes. We then developed and compared two distinct mathematical/statistical approaches for building our internet traffic models; one model is computationally faster but less accurate, while the other is more computationally expensive but can capture extreme points seen in the data. Thus, depending on the desired accuracy and computational power, users can utilize our models to generate artificial internet traffic that takes into account the number of users and the activities performed (e.g. web-browsing, video streaming, online gaming). 
Print Edition ISSN 1536-4585

Online Edition ISSN 2375-8732 